\title{
WestVirginiaUniversity
}

THE RESEARCH REPOSITORY @ WVU

Graduate Theses, Dissertations, and Problem Reports

2020

\section{Cycle Double Covers and Integer Flows}

\author{
Zhang Zhang \\ West Virginia University, zazhang@mix.wvu.edu
}

Follow this and additional works at: https://researchrepository.wvu.edu/etd

Part of the Discrete Mathematics and Combinatorics Commons

\section{Recommended Citation}

Zhang, Zhang, "Cycle Double Covers and Integer Flows" (2020). Graduate Theses, Dissertations, and Problem Reports. 7646.

https://researchrepository.wvu.edu/etd/7646

This Dissertation is protected by copyright and/or related rights. It has been brought to you by the The Research Repository @ WVU with permission from the rights-holder(s). You are free to use this Dissertation in any way that is permitted by the copyright and related rights legislation that applies to your use. For other uses you must obtain permission from the rights-holder(s) directly, unless additional rights are indicated by a Creative Commons license in the record and/ or on the work itself. This Dissertation has been accepted for inclusion in WVU Graduate Theses, Dissertations, and Problem Reports collection by an authorized administrator of The Research Repository @ WVU.

For more information, please contact researchrepository@mail.wvu.edu. 


\title{
Cycle Double Covers and Integer Flows
}

\author{
Zhang Zhang \\ Dissertation submitted to the \\ Eberly College of Arts and Sciences \\ at West Virginia University \\ in partial fulfillment of the requirements \\ for the degree of \\ Doctor of Philosophy \\ in \\ Mathematics \\ Cun-Quan Zhang, Ph.D., Chair \\ John Goldwasser, Ph.D. \\ Hong-Jian Lai, Ph.D. \\ James Nolan, Ph.D. \\ Jerzy Wojciechowski, Ph.D. \\ Department of Mathematics \\ West Virginia University \\ Morgantown, West Virginia \\ 2020
}

Keywords: Integer flows, Cycle double covers, Face coloring problems, Signed graphs, Bouchet's conjecture, embedded graphs

Copyright 2020 Zhang Zhang 


\section{ABSTRACT \\ Cycle Double Covers and Integer Flows}

\section{Zhang Zhang}

My research focuses on two famous problems in graph theory, namely the cycle double cover conjecture and the integer flows conjectures. This kind of problem is undoubtedly one of the major catalysts in the tremendous development of graph theory. It was observed by Tutte that the Four color problem can be formulated in terms of integer flows, as well as cycle covers. Since then, the topics of integer flows and cycle covers have always been in the main line of graph theory research. This dissertation provides several partial results on these two classes of problems.

Fleischner's problem concerning the compatible circuit decomposition is solved in Chapter 1 , which is closely related to the famous cycle double cover conjecture. Actually, a compatible circuit decomposition is basically a circuit decomposition of an eulerian graph satisfying the required properties. Such a decomposition implies the existence of a cycle double cover in the following way: Let $G$ be an arbitrarily bridgeless graph and $\tilde{G}$ be the eulerian graph obtained from $G$ by replacing each edge with a pair of parallel edges. Since $\tilde{G}$ is an eulerian graph, it has a circuit decomposition. We further require that each pair of parallel edges can't occur in the same circuit. This is just a special case of compatible circuit decomposition. Clearly, such a decomposition corresponds to a cycle double cover of the original graph G. Fleischner (1990's) wondered implicitly whether if an even graph does not have a compatible circuit decomposition then it must have an undecomposable $K_{5}$-transition-minor or its generalized transition-minor. This conjecture is now completely solved in this paper.

The Four color conjecture can be viewed as a coloring problem on orientable surfaces. Indeed, Tutte showd the equivalence of face coloring problem and the integer flows problems. In Chapter 2 , we further generalize it into non-orientable surfaces by introducing the natural signatures on signed graphs. In 1983, Bouchet conjectured that every flow-admissible signed graph admits a nowhere-zero 6-flow. In Chapter 2, we deduce this conjecture to a small class of graphs by applying the classification theorem of surfaces. Moreover, we verify this conjecture for a special class of embedding graphs.

In Chapter 3, we show that every flow-admissible signed graph admits a nowhere-zero 11flow, which is the best partial result to Bouchet's conjecture. The main part is to prove that every flow-admissible signed graph admits a balanced nowhere-zero $\mathbb{Z}_{2} \times \mathbb{Z}_{3}$-flow, which will be a powerful tool in dealing with the integer flow problems of signed graphs. We also discuss the conversion of modulo flows into integer flows in this Chapter. In particular a new result to convert a modulo 3 -flow to an integer 5-flow will be introduced and proved. 


\section{Acknowledgements}

First and foremost, I am most indebted to my supervisor, Dr. Cun-quan Zhang, for his continued encouragement and support over these last few years. It is a pleasure to work under his supervision. Without him, this dissertation could not have come about.

I would like to thank my other committee members: Dr. John Goldwasser, Dr. Hong-jian Lai, Dr. James Nolan, and Dr. Jerzy Wojciechowski, for their help during my studies. My thanks also goes to all the professors who have given me support and help in my study and in my daily life.

Finally, I would like to thank the Department of Mathematics and Eberly College of Arts and Sciences at West Virginia University for providing me with an excellent study environment and support during my study as a graduate student. 


\section{DEDICATION}

To

my father Yong-li Zhang, my mother Yong-jun Li, 


\section{Contents}

$1 \operatorname{Bad} K_{5} \quad 1$

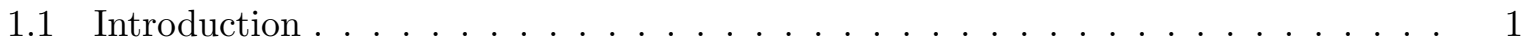

1.2 Preliminary Discussions . . . . . . . . . . . . . . . . . 3

1.3 Main results . . . . . . . . . . . . . . . . . . 8

1.4 Primary lemmas . . . . . . . . . . . . . . . . . . . 9

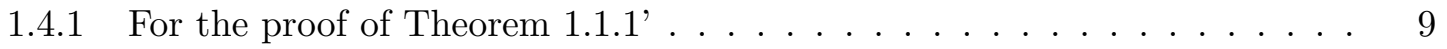

1.4.2 Cornered triangle extension property: key lemmas for the determination of $\mathrm{UD}-K_{5} \ldots \ldots \ldots \ldots \ldots$

1.5 Simultaneous proof of Theorems 1.1.1' and $1.1 .2 \ldots \ldots \ldots$

1.5.1 Case I. $(G, \mathcal{T})$ is a counterexample to Theorem 1.1.1' . . . . . . . . 21

1.5.2 Case II. $(G, \mathcal{T})$ is a counterexample to Theorem 1.1.2. . . . . . . . . . 25

2 Embedding signed flows $\quad 31$

2.1 Introduction . . . . . . . . . . . . . . . . . . 31

2.2 Main result: the methods of surfaces operations . . . . . . . . . . . . . 32

2.2.1 Notation and terminology . . . . . . . . . . . . . . . . . 32

2.2.2 Face coloring for non-orientable surfaces . . . . . . . . . . . . . . . . . . . . . . . 33

2.2 .3 topological methods . . . . . . . . . . . . . . . 35

2.2 .4 Switch when cut-paste . . . . . . . . . . . . . . . . . 39

2.3 Twin propeller graphs . . . . . . . . . . . . . . . . . . . . . 41

3 11-FLOW $\quad 46$

3.1 Introduction . . . . . . . . . . . . . . . . . . . 46

3.2 Signed graphs, switch operations, and flows . . . . . . . . . . . . . . 47

3.3 Modulo flows on signed graphs . . . . . . . . . . . . . . . . 48

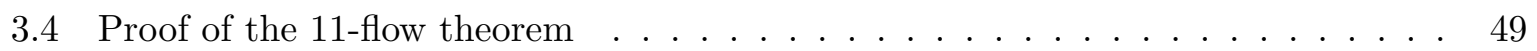

3.5 Proof of Theorem $3.3 .2 \ldots \ldots \ldots \ldots$

3.6 Proof of Theorem $3.1 .3 \ldots \ldots \ldots \ldots \ldots \ldots \ldots \ldots$ 
3.6.1 Shrubberies . . . . . . . . . . . . . . . . . 52

3.6 .2 Nowhere-zero watering . . . . . . . . . . . . . . . . 55

3.6.3 Completing the proof of Theorem $3.1 .3 \ldots \ldots \ldots \ldots$. . . . . . 67 


\section{Chapter 1}

\section{$\operatorname{Bad} K_{5}$}

The well known cycle double cover conjecture, proposed independently by Tutte and other mathematicians, states that every bridgeless graph has a collection of cycles which together cover each edge of the graph exactly twice.

The following is an approach towards the cycle double cover conjecture, using circuit decomposition of eulerian graphs: Let $G$ be arbitrarily a bridgeless graph and $\tilde{G}$ be the eulerian graph obtained from $G$ by replacing each edge with a pair of parallel edges. Since $\tilde{G}$ is an eulerian graph, it has a circuit decomposition. We further require that each pair of parallel edges can not occur in the same circuit. This is just a special case of compatible circuit decomposition. Clearly, such a decomposition corresponds to a cycle double cover of the original graph $G$. This relation leads us the following problem.

\subsection{Introduction}

Compatible Circuit Decomposition (CCD) Problem. Let $G$ be a 2-connected eulerian graph with $\delta(G) \geq 4$, and for each $v \in V(G)$ let $\mathcal{T}(v)$ be a set of edge-disjoint edge-pairs (called transitions) of $E(v)$ (in the case of a loop $l$ we allow $\{l, l\}$ to be a transition). Can we find a circuit decomposition $\mathcal{C}$ of $G$ such that, for every $C \in \mathcal{C}$ and every $v \in V(G)$ and every $P \in \mathcal{T}(v)$, $|E(C) \cap P| \leq 1$ (unless $C$ is a loop and $P=\{l, l\}$, in which case there is no CCD)?

Such $\mathcal{C}$ is called compatible with the transition system $\mathcal{T}=\bigcup_{v \in V(G)} \mathcal{T}(v)$ (see also Definition 1.2.2).

The compatible circuit decomposition (CCD) problem is closely related to the famous circuit double cover conjecture, $[16,24,27,30]$, and to the Sabidussi conjecture $[11,12]$.

It is well known that not every eulerian graph associated with a transition system has a compatible circuit decomposition. For example, an undecomposable $K_{5}$ (or, a bad $K_{5}$ to use a 
more colloquial expression) is the complete graph $K_{5}$ associated with the transition system

$$
\mathcal{T}_{5}=\left\{\left\{v_{i} v_{i+\mu}, v_{i} v_{i-\mu}\right\}: i \in \mathbb{Z}_{5}, \mu \in\{1,2\}\right\}
$$

where $V\left(K_{5}\right)=\left\{v_{0}, v_{1}, \ldots, v_{4}\right\}$ (see Figure 1.1).

The compatible circuit decomposition problem has been verified for planar graphs by Fleischner [11], and for $K_{5}$-minor-free graphs by Fan and Zhang [9]. Fleischner further asked implicitly the following question [14] which is beyond a graph-minor problem. In what follows we restrict ourselves to 2-connected graphs.

Problem 1. (Fleischner [14]) If $(G, \mathcal{T})$ does not have a compatible circuit decomposition, does $(G, \mathcal{T})$ contain either an undecomposable $K_{5}$-transition-minor or one of its generalized transitionminors?

A transition-minor is not only a graph-minor that preserves some topological structure of $G$ but also inherits the original transition system $\mathcal{T}$ (see Definitions 1.2.7 and 1.2.9 for definitions of transition-minor and SUD- $K_{5}$ ). Problem 1 is completely solved in this chapter.

Theorem 1.1.1. Let $(G, \mathcal{T})$ be a 2-connected eulerian graph with the minimum degree $\delta \geq 4$ associated with a transition system. If $(G, \mathcal{T})$ is SUD-K-minor-free, then it has a compatible circuit decomposition.

We observe that if $\mathcal{T}=\emptyset$, then any circuit decomposition of $(G, \mathcal{T})$ is in accordance with Theorem 1.1.1. Thus, we assume that our point of departure is a $(G, \mathcal{T})$ with $\mathcal{T} \neq \emptyset$.

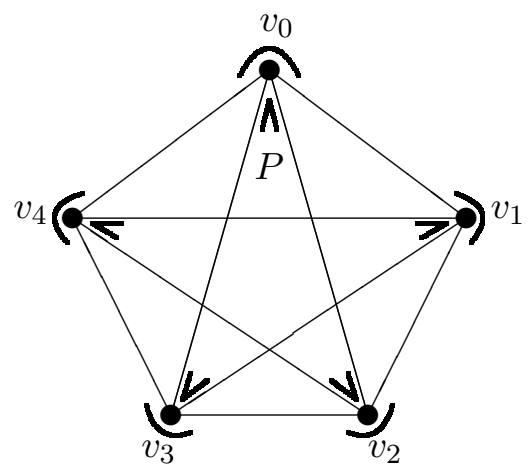

Figure 1.1: $K_{5}$ with $\mathcal{T}_{5}=\left\{\left\{v_{i-1} v_{i}, v_{i} v_{i+1}\right\},\left\{v_{i-2} v_{i}, v_{i} v_{i+2}\right\}: i \in \mathbb{Z}_{5}\right\}$

In the study of circuit cover and circuit decomposition problems, one of the fundamental steps is to determine the structure of two adjacent circuits (i.e., two circuits having at least one vertex in common). The Hamilton weight problem ( $[20,34])$ is one of such approaches for faithful cover problem. Its corresponding version for circuit decomposition is the Hamilton transition problem. That is, if $(G, \mathcal{T})$ has some compatible circuit decomposition and every 
such decomposition consists of a pair of hamiltonian circuits, then $(G, \mathcal{T})$ must be constructed recursively from two loops $(2 L)$ via a series of $(X \leftrightarrow O)$-operations (the operation extending a vertex to a digon); see Definition 1.2.13 and Conjecture A. The family of transitioned graphs constructed in such a way is denoted by $\langle 2 L\rangle$. This problem is solved in this paper for SUD$K_{5}$-minor-free graphs, as stated in Theorem 1.1.2 below.

Theorem 1.1.2. Let $(G, \mathcal{T})$ be a 4-regular fully transitioned graph that has a compatible circuit decomposition and such that every such decomposition consists of a pair of hamiltonian circuits. If $(G, \mathcal{T})$ is SUD- $K_{5}$-minor-free, then $(G, \mathcal{T}) \in\langle 2 L\rangle$.

This result plays a key role in the determination of a UD- $K_{5}$-transition-minor in Theorem 1.1.1. It is important to point out that both Theorems 1.1.1 and 1.1.2 are proved simultaneously because one provides the structures of extreme cases, while the other assures the existence of a compatible circuit decomposition for any proper minor of a smallest counterexample.

The rest of the chapter is organized as follows. Some notation and terminology are recalled and introduced in Section 1.2. Main results, Theorems 1.1.1 and 1.1.2 are further summarized in Section 1.3. In Section 1.4, some preliminary lemmas for Theorem 1.1.1 are proved in Subsection 1.4.1 before its simultaneous proof with Theorem 1.1.2 (in Section 1.5). There are other important results (Lemmas 1.4.10 and 1.4.11) in Subsection 1.4.2 that determine the specific structure of UD- $K_{5}$ and are used in the simultaneous proof of Theorems 1.1.1 and 1.1.2.

\subsection{Preliminary Discussions}

For terminology and notation not defined here we follow $[4,7,32]$, and the papers listed in the References.

A circuit is a 2-regular connected subgraph of a given graph $G$. A subgraph $H$ of $G$ is called even or eulerian if $\operatorname{deg}_{H}(v)$ is even for every vertex $v \in V(H)$.

Let $v$ be a degree two vertex of a given graph $G$. Suppressing $v$ is the operation of removing $v$ and adding an edge between the two neighbours of $v$ in $G$.

Definition 1.2.1. A vertex subset $U$ is a separator of $G$ separating $G$ to $G_{1}, G_{2}$ if $E(G)=$ $E\left(G_{1}\right) \cup E\left(G_{2}\right)$ and $V\left(G_{1}\right) \cap V\left(G_{2}\right)=U$ and $E\left(G_{1}\right) \cap E\left(G_{2}\right)=\emptyset$. $U$ is a t-separator if $|U|=t$. We say a separator $U$ separating subgraphs $X_{1}, X_{2}$ of $G$ if $U$ is a separator of $G$ separating $G$ to $G_{1}, G_{2}$ with $X_{i} \subseteq G_{i}, i=1,2$.

Definition 1.2.2. Let $G$ be an eulerian graph, and, for each $v \in V(G)$ with $\operatorname{deg}(v) \geq 4$, let $\mathcal{T}(v)$ be a set of edge-disjoint edge-pairs of $E(v)$. The set $\mathcal{T}=\bigcup_{v \in V(G)} \mathcal{T}(v)$ is called a transition system of $G$ and each member of $\mathcal{T}$ is called a transition. A non-trivial vertex is a vertex with 
some transition (that is, $\mathcal{T}(v) \neq \emptyset$ ); otherwise, we called $v$ a trivial vertex. The graph $G$ with a transition system $\mathcal{T}$ is called a transitioned graph and denoted by $(G, \mathcal{T}) ;($ possibly $\mathcal{T}=\emptyset) . A$ fully transitioned graph is a transitioned graph without trivial vertex. For every subgraph $H$ of $G,\left.\mathcal{T}\right|_{H}=\{P \in \mathcal{T} \mid P \subset E(H)\}$. In the case of multiple edges e, $f$ at $u, v \in V(G)$, we distinguish between the transition $\{e, f\}$ at $u$ and the transition $\{e, f\}$ at $v$.

Definition 1.2.3. Let $(G, \mathcal{T})$ be a transitioned graph.

(1) A 1-separator $\{v\}$ separating $G$ to $G_{1}, G_{2}$ is a bad cut-vertex if $E(v) \cap E\left(G_{i}\right) \in \mathcal{T}$ for at least one $i \in\{1,2\}$.

(2) $(G, \mathcal{T})$ is admissible if it does not have a bad cut-vertex.

Definition 1.2.4. Let $(G, \mathcal{T})$ be a transitioned graph. Let $C=v_{0} v_{1} \ldots v_{r-1} v_{0}$ be a circuit. Let $e_{i}$ be the edge of $C$ joining $v_{i}$ and $v_{i+1}$ for every $i \in \mathbb{Z}_{r}$.

(1) $v_{i}$ is an inner vertex of $C$ if $\left\{e_{i-1}, e_{i}\right\} \in \mathcal{T}\left(v_{i}\right)$ or $E\left(v_{i}\right) \backslash\left\{e_{i-1}, e_{i}\right\} \in \mathcal{T}\left(v_{i}\right)$, and we call $\left\{e_{i-1}, e_{i}\right\}$ an inner transition of $C$ at $v_{i} . C$ is compatible at $v_{i}$ if it is not an inner vertex of $C$.

(2) $C$ is a compatible circuit of $(G, \mathcal{T})$ if $C$ is compatible at every vertex of $C$.

Definition 1.2.5. A family $\mathcal{F}$ of circuits of $G$ is a compatible circuit decomposition (abbreviated $\mathrm{CCD})$ of $(G, \mathcal{T})$ if $\mathcal{F}$ is a circuit decomposition of $G$ and every member of $\mathcal{F}$ is a compatible circuit.

It is obvious that the absence of bad cut-vertices (see Definition 1.2.3) is a necessary condition for a transitioned graph admitting a CCD.

Observation 1.2.1. Consider a non-trivial vertex $v$ of degree 4 in $(G, \mathcal{T})$. Let $E(v)=\left\{e_{1}, \ldots, e_{4}\right\}$ and $P=\left\{e_{1}, e_{2}\right\} \in \mathcal{T}(v)$. Then every circuit of a CCD of $(G, \mathcal{T})$ covers at most one edge of $\left\{e_{3}, e_{4}\right\}$. This means in a natural way and without loss of generality, we can assume that if $P \in \mathcal{T}(v)$, then $E(v) \backslash P \in \mathcal{T}(v)$, for every vertex $v$ of degree 4 . Thus every vertex $v$ of degree 4 is either a trivial vertex, or $|\mathcal{T}(v)|=2$.

Definition 1.2.6. A circuit $C$ is a removable circuit of $(G, \mathcal{T})$ if it is compatible and $(G \backslash$ $\left.E(C),\left.\mathcal{T}\right|_{G \backslash E(C)}\right)$ remains admissible (that is, $\left(G \backslash E(C),\left.\mathcal{T}\right|_{G \backslash E(C)}\right)$ has no bad cut-vertex).

Definition 1.2.7. Let $(G, \mathcal{T})$ be a transitioned eulerian graph, and, $G^{\prime}=\left(G \backslash F_{d}\right) / F_{c}$ be an eulerian minor of $G$ obtained by deleting $F_{d}$ and contracting $F_{c}$ where $F_{d}, F_{c} \subseteq E(G)$. The resulting transition system $\mathcal{T}^{\prime}=\left.\mathcal{T}\right|_{G^{\prime}}$ on $G^{\prime}$ is defined as follows.

(1) Delete the edges of $\left(F_{d} \cup F_{c}\right)$. The resulting transition system $\mathcal{T}^{\prime}$ contains all transitions $P \in \mathcal{T}$ for which $P \subseteq E\left(G \backslash\left(F_{d} \cup F_{c}\right)\right)$. 
(2) For each edge $e=v_{e}^{\prime} v_{e}^{\prime \prime} \in F_{c}$, identify the end-vertices $v_{e}^{\prime}$ and $v_{e}^{\prime \prime}$ as a new vertex $v_{e}$.

(3) Since we do not define a transition at any vertex $v$ of degree $2, \mathcal{T}^{\prime}(v)=\emptyset$ if $\operatorname{deg}_{G^{\prime}}(v)=2$. And we apply Observation 1.2.1 to extend $\mathcal{T}^{\prime}(z)$ if $\operatorname{deg}_{G^{\prime}}(z)=4$.

The resulting transitioned graph $\left(G^{\prime}, \mathcal{T}^{\prime}\right)$ is called a transition-minor of $(G, \mathcal{T})$.

Definition 1.2.8. $(G, \mathcal{T})$ is called the undecomposable $K_{5}$ (UD- $K_{5}$ for short) if $G=K_{5}$, and the transition system $\mathcal{T}$ is defined as follows.

$$
\mathcal{T}\left(v_{i}\right)=\left\{\left\{v_{i} v_{i+\mu}, v_{i} v_{i-\mu}\right\}: \mu \in\{1,2\} \quad(\bmod 5)\right\}
$$

for every $v_{i} \in V\left(K_{5}\right)=\left\{v_{0}, v_{1}, \ldots, v_{4}\right\}$; see Figure 1.1 .

Definition 1.2.9. The transitioned graph $(G, \mathcal{T})$ is a sup-undecomposable $K_{5}$ (SUD- $K_{5}$ for short) if the graph $G$ can be decomposed into 15 connected edge-disjoint subgraphs

$$
\left\{P_{i, j}:\{i, j\} \subset \mathbb{Z}_{5}, i<j\right\} \cup\left\{Q_{i}: i \in \mathbb{Z}_{5}\right\}
$$

as follows (see Figure 1.2).

(1) Each $P_{i, j}$ is a path joining $V\left(Q_{i}\right)$ and $V\left(Q_{j}\right)(i<j)$, and the different $P_{i, j}$ 's are internally disjoint;

(2) $\left\{Q_{i}: i \in \mathbb{Z}_{5}\right\}$ are disjoint connected subgraphs;

(3) Let $Q_{i}^{+}$be the subgraph of $H$ induced by $E\left(Q_{i}\right)$ and the four adjacent paths $P_{i, j}$ (for every pair $j \neq i)$. Then each subgraph $Q_{i}^{+}$has a bad cut-vertex $u_{i}$ separating $P_{i,(i+1)} \cup P_{i,(i-1)}$ and $P_{i,(i+2)} \cup P_{i,(i-2)}$, where $u_{i} \in V\left(Q_{i}\right)$.

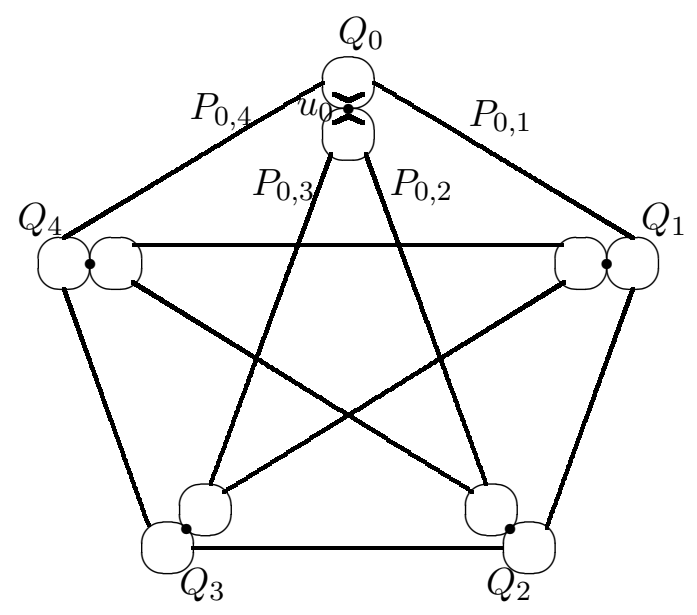

Figure 1.2: A sup-undecomposable $K_{5}$

Note that a UD- $K_{5}$ is a special case of a SUD- $K_{5}$ where $\left|Q_{i}\right|=1$ for every $i \in \mathbb{Z}_{5}$. 
Definition 1.2.10. $(G, \mathcal{T})$ is sup-undecomposable $K_{5}$-transition-minor free (or, SUD- $K_{5}$-minorfree for short) if it does not have any eulerian minor $H$ such that $\left(H,\left.\mathcal{T}\right|_{H}\right)$ is a SUD- $K_{5}$.

The following is a straightforward observation.

Observation 1.2.2. Let $G^{\prime}$ be an eulerian minor of $G$. If $(G, \mathcal{T})$ is SUD-K $K_{5}$-minor-free, then $\left(G^{\prime}, \mathcal{T}^{\prime}\right)$ remains SUD-K $K_{5}$-minor-free (where $\mathcal{T}^{\prime}$ is described in Definition 1.2.7).

Example 1. In [15], an infinite family of snarks $\left\{H_{n}\right\}$ has been constructed, which has a 2-factor $F_{n}$ such that $F_{n}$ is not contained in any circuit double cover of $H_{n}$. Let $\overline{H_{n}}$ be the 4-regular graph obtained from $H_{n}$ by contracting the 1 -factor $H_{n} \backslash F_{n}$ and $\mathcal{T}_{n}$ be the transition system of $\overline{H_{n}}$ such that each circuit of $F_{n}$ has all its vertices as inner vertices (see Definition 1.2.4(1)). Clearly, $\left(\overline{H_{n}}, \mathcal{T}_{n}\right)$ has no CCD. Otherwise we can get a circuit double cover by taking $F_{n}$ together with the CCD of $\left(\overline{H_{n}}, \mathcal{T}_{n}\right.$ ) (after a proper adjustment by adding edges of $H_{n} \backslash F_{n}$ ). The 4-regular graph illustrated in Figure $1.3-(a)$ is the contracted graph $\overline{H_{0}}$ where the 2-factor $F_{0}$ is a pair of edge-disjoint hamiltonian circuits (illustrated by thin lines and thick lines). A study in [15] reveals that each member $\left(\overline{H_{n}}, \mathcal{T}_{n}\right)$ in this family contains a UD- $K_{5}$-minor due to the structure of $\left(\overline{H_{n}}, \mathcal{T}_{n}\right)$. For example, the resulting transition graph by deleting some edges $\overline{H_{0}}$ is a subdivision of a UD- $K_{5}$ (illustrated in Figure 1.3-(b)). Therefore, every transitioned 4-regular graph $\left(\overline{H_{n}}, \mathcal{T}_{n}\right)$ in this family contains a SUD- $K_{5}$-minor and does not have a CCD.

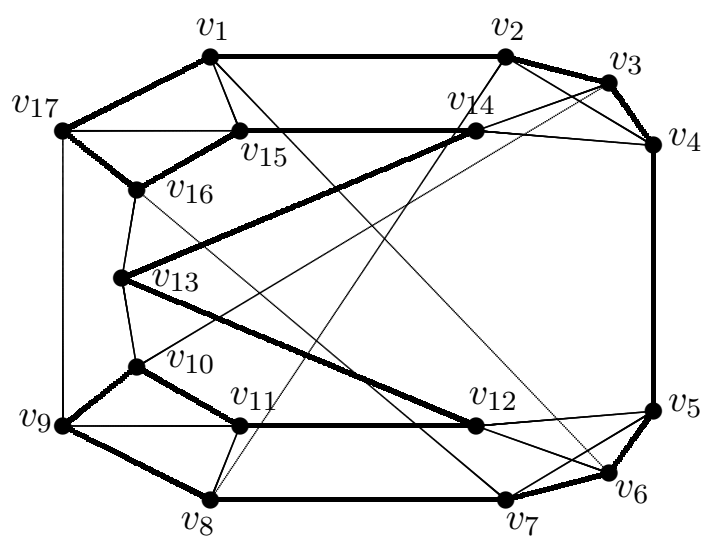

Figure 1.3: $(a)\left(\overline{H_{0}}, \mathcal{T}_{0}\right)$ has no CCD.

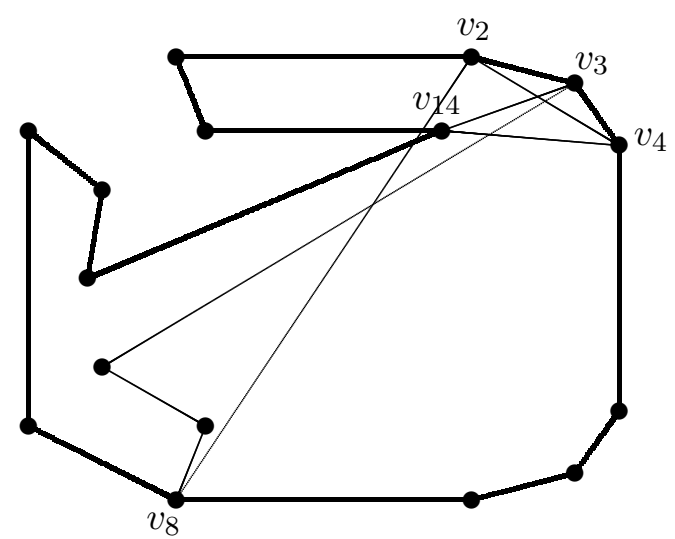

(b) A UD- $K_{5}-$ minor in $\left(\overline{H_{0}}, \mathcal{T}_{0}\right)$.

Next, we introduce the Hamiltonian circuit decomposition problem, which is the corresponding Hamilton weight problem in faithful circuit cover.

Definition 1.2.11. Let $(G, \mathcal{T})$ be a fully transitioned 4-regular graph. If every $\mathrm{CCD}$ of $(G, \mathcal{T})$ is a pair of hamiltonian circuits, then $(G, \mathcal{T})$ is called a Hamilton transitioned graph. 
Definition 1.2.12. Let $D=v_{0} v_{1} v_{0}$ be a digon. $D$ is of type $\lambda$ where $\lambda$ is the number of inner vertices of $D$ (see Figure 1.4).
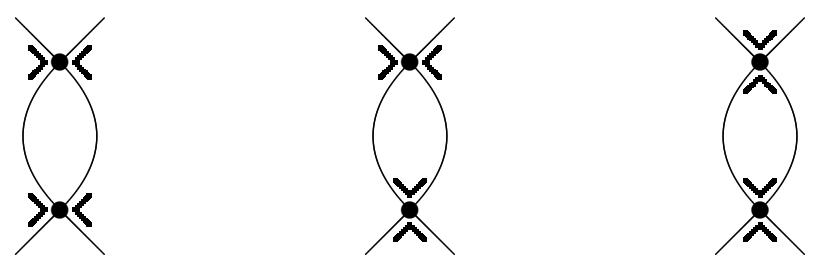

Figure 1.4: Digons of type 0,1 , and 2, respectively.

Definition 1.2.13. Let $v$ be a non-trivial degree 4 vertex of a transitioned graph $(G, \mathcal{T})$. The $(X \leftrightarrow O)$-operation at $v$ with $\mathcal{T}(v)=\left\{\left\{e_{1}, e_{2}\right\},\left\{e_{3}, e_{4}\right\}\right\}$ is defined as follows (see Figure 1.5). Split $v$ with $\left\{e_{1}, e_{2}\right\}$ becoming incident to a new vertex $v_{1}$ and $\left\{e_{3}, e_{4}\right\}$ incident to another new vertex $v_{2}$, and add a pair of parallel edges $\left\{e_{5}, e_{6}\right\}$ between $v_{1}$ and $v_{2}$, and define a new transition system by replacing $\mathcal{T}(v)$ with $\mathcal{T}\left(v_{2}\right)=\left\{\left\{e_{3}, e_{4}\right\},\left\{e_{5}, e_{6}\right\}\right\}$ and with either $\mathcal{T}\left(v_{1}\right)=$ $\left\{\left\{e_{1}, e_{5}\right\},\left\{e_{2}, e_{6}\right\}\right\}$ or $\mathcal{T}\left(v_{1}\right)=\left\{\left\{e_{1}, e_{2}\right\},\left\{e_{5}, e_{6}\right\}\right\}$. In fact, we have created a digon of type $>0$ between $v_{1}$ and $v_{2}$.

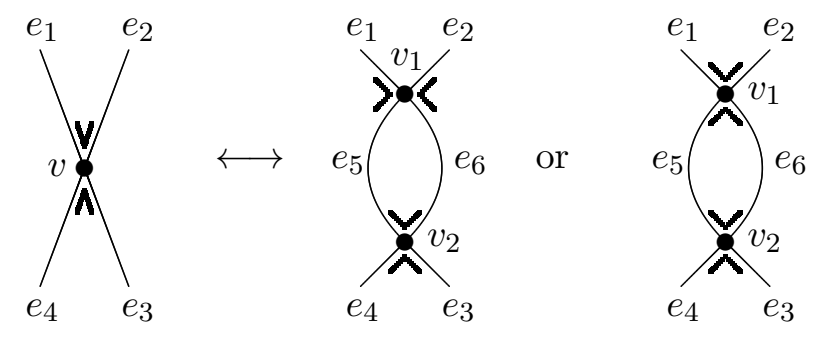

Figure 1.5: $(X \leftrightarrow O)$-operations.

Definition 1.2.14. Denote by $\langle 2 L\rangle$ the family of all transitioned 4-regular graphs obtained from $\left(2 L, \mathcal{T}_{2}\right)$ (which appears on the top left of Figure 1.6) by a sequence of $(X \leftrightarrow O)$-operations; it is called the $2 L$-family and its members are called $\langle 2 L\rangle$-elements.

Lemma 1.2.1. Let $(G, \mathcal{T}) \in\langle 2 L\rangle$ be of order at least 3 . Then $(G, \mathcal{T})$ has either two vertexdisjoint digons of type $\geq 1$, or two edge-disjoint digons of type $\geq 1$ with at least one inner transition in the common vertex. 

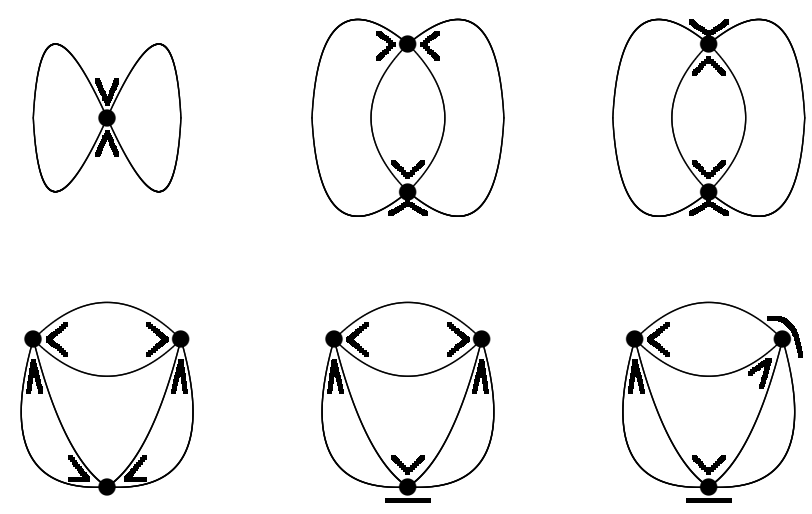

Figure 1.6: $\langle 2 L\rangle$-elements of order $\leq 3$.

Proof. Note that the order of $(G, \mathcal{T}) \in\langle 2 L\rangle$ being at least 3 implies that $G$ does not contain an edge with multiplicity more than 2 (this is straightforward from the definition of $\langle 2 L\rangle$ ). The family $\langle 2 L\rangle$ has precisely three members of order 3 (see Figure 1.6); in this case, every $(G, \mathcal{T}) \in\langle 2 L\rangle$ has two edge-disjoint digons of type $>0$ sharing a common inner vertex.

Thus, the statement of the lemma is true for $(G, \mathcal{T}) \in\langle 2 L\rangle$ of order 3 . Hence suppose that $G$ is of order greater than 3 .

Since $(X \leftrightarrow O)$-operations create a new digon of type $>0$, every member of $\langle 2 L\rangle$ except $2 L$ contains at least one digon of type $>0$. Let $D$ be a digon of type $\lambda>0$ in $(G, \mathcal{T})$ and let $\left(G^{\prime}, \mathcal{T}^{\prime}\right) \in\langle 2 L\rangle$ be the graph obtained from $(G, \mathcal{T})$ by contracting $D$. By induction on $|V(G)|$, $\left(G^{\prime}, \mathcal{T}^{\prime}\right)$ has either two vertex-disjoint digons of type $>0$ or two edge-disjoint digons of type $>0$ with an inner transition in a common vertex in each of these two digons. In all cases at least one of these digons of type $>0$ and $D$ are either two vertex-disjoint digons of type $>0$ or two edge-disjoint digons of type $>0$ with inner transitions in the common vertex in $(G, \mathcal{T})$.

\subsection{Main results}

Given Definition 1.2.3, Theorem 1.1.1 is restated as a stronger version below.

Theorem 1.1.1'. Let $(G, \mathcal{T})$ be an eulerian graph associated with an admissible transition system. If $(G, \mathcal{T})$ is SUD- $K_{5}$-minor-free, then it has a CCD.

Theorem 1.1.1' is not only a graph minor problem, but also a transition minor problem. It was originally proposed by Fleischner [14]. Its weak version for graph minors was solved by Fleischner [11] for planar graphs, and by Fan and Zhang [9] for $K_{5}$-minor-free graphs.

Note that Theorem 1.1.1' is stronger than the following theorem which is only a graph-minor- 
free result (not a transition-minor-free result).

Theorem A. [9] Let $\mathcal{T}$ be an admissible transition system of an eulerian graph $G$. Then $(G, \mathcal{T})$ has a CCD if $G$ is $K_{5}$-minor-free.

In the studies of circuit covering problems or circuit decomposition problems, one of the critical steps is to determine the structure of the subgraph induced by a pair of incident circuits ( $[36,38]$, etc.). The structure of a graph that is covered by or decomposed into a pair of hamiltonian circuits provides a local structure of a possible counterexample to many open problems (such as the circuit double cover conjecture). Its structure for the faithful circuit covering problem was conjectured in [34]; the following is an equivalent version for the corresponding compatible circuit decomposition problem.

Conjecture A. [34] Let $(G, \mathcal{T})$ be a fully transitioned 4-regular graph such that it has some $\mathrm{CCD}$ and every such decomposition consists of a pair of hamiltonian circuits. Then $(G, \mathcal{T}) \in\langle 2 L\rangle$.

Theorem 1.1.2 solves Conjecture A for SUD- $K_{5}$-minor-free graphs. This result generalizes an early result by Lai and Zhang [20] which is a graph minor result for the faithful covering problem.

Note that, in this paper, Theorems 1.1.1' and 1.1.2 are proved simultaneously, which indicates the technical importance of Hamilton transitioned results (such as, Theorem 1.1.2) in the studies of this area.

\subsection{Primary lemmas}

\subsubsection{For the proof of Theorem 1.1.1'}

We consider a counterexample $(G, \mathcal{T})$ to Theorem 1.1.1', such that

(1) $|E(G)|$ is as small as possible;

(2) subject to (1), the number of transitions is as small as possible.

$(G, \mathcal{T})$ is called a smallest counterexample to Theorem 1.1.1'. It follows from the choice of $(G, \mathcal{T})$ that $(G, \mathcal{T})$ has no removable circuit.

Definition 1.4.1. Let $v$ be a non-trivial vertex in a transitioned 4 -regular graph $(G, \mathcal{T}) . A$ circuit decomposition of $(G, \mathcal{T})$ is called an almost compatible circuit decomposition with respect to $v$, if it is compatible in every vertex except $v$.

A sequence of edge-disjoint circuits $\left\{C_{1}, \ldots, C_{k}\right\}(k \geq 2)$ of $(G, \mathcal{T})$ is called an almost compatible circuit chain decomposition with respect to $v(\operatorname{ACCCD}(v)$ for short $)$, if 
(1) it is an almost compatible circuit decomposition with respect to $v$;

(2) $v \in V\left(C_{1}\right) \cap V\left(C_{k}\right)$, and $v \notin V\left(C_{i}\right) \forall i \in\{2, \ldots, k-1\}$.

(3) for each $i, j \in\{1, \ldots, k\}$ with $i \neq j,\left[V\left(C_{i}\right) \cap V\left(C_{j}\right)\right] \backslash\{v\} \neq \emptyset$ if and only if $|j-i|=1$.

The integer $k$ is called the length of the chain $\left\{C_{1}, \ldots, C_{k}\right\}$ (see Figure 1.7).

By an approach similar to the one in [2], [1] and [9], we obtain the following structural results. For the purpose of being self-contained, proofs are therefore included.

Lemma 1.4.1. [9] Let $(G, \mathcal{T})$ be a smallest counterexample to Theorem 1.1.1' and let $\mathcal{F}_{v}=$ $\left\{C_{1}, \ldots, C_{k}\right\}$ be an ACCCD of $(G, \mathcal{T})$ with respect to a non-trivial vertex $v$. If $k \geq 3$, then $V\left(C_{1}\right) \cap V\left(C_{k}\right)=\{v\}$.

Proof. By Definition 1.4.1, $v \in V\left(C_{1}\right) \cap V\left(C_{k}\right)$. Let $H$ be the subgraph induced by $E\left(C_{1}\right) \cup E\left(C_{k}\right)$. If $\left|V\left(C_{1}\right) \cap V\left(C_{k}\right)\right| \geq 2$, then $\left(H,\left.\mathcal{T}\right|_{H}\right)$ is 2 -connected. So each $C_{i}, 1<i<k$, is a removable circuit, which is a contradiction.

Lemma 1.4.2. [9] Any smallest counterexample $(G, \mathcal{T})$ to Theorem 1.1.1' is 4-regular, 2connected, and for every non-trivial vertex $v$ of $(G, \mathcal{T})$, there exists an $\operatorname{ACCCD}(v)$. Furthermore, every almost $C C D$ with respect to $v$ is an $\operatorname{ACCCD}(v)$.

Proof. Let $(G, \mathcal{T})$ be a smallest counterexample to Theorem 1.1.1'. Since $\mathcal{T}$ is admissible, $(G, \mathcal{T})$ has no bad cut-vertex. If $\{v\}$ is a 1-separator of $G$ separating $G$ to $G_{1}, G_{2}$, then $\left(G_{1},\left.\mathcal{T}\right|_{G_{1}}\right)$ and $\left(G_{2},\left.\mathcal{T}\right|_{G_{2}}\right)$ have CCD's $\mathcal{C}_{1}$ and $\mathcal{C}_{2}$, respectively, Thus, $\mathcal{C}_{1} \cup \mathcal{C}_{2}$ is a CCD of $(G, \mathcal{T})$, a contradiction. Therefore, $G$ is 2 -connected.

Let $v$ be a non-trivial vertex in $G$ and let $\left(G^{\prime}, \mathcal{T}^{\prime}\right)$ be a transitioned graph obtained from $(G, \mathcal{T})$ by removing one transition in vertex $v$, if $\operatorname{deg}(v)>4$, or by removing all transitions of $\mathcal{T}(v)$, if $\operatorname{deg}(v)=4$.

By the choice of $(G, \mathcal{T})$, the new graph $\left(G^{\prime}, \mathcal{T}^{\prime}\right)$, which has a smaller number of transitions, has a $\mathrm{CCD}, \mathcal{F}_{v}$. Let $C_{v}$ be the circuit of $\mathcal{F}_{v}$ containing the vertex $v$ and one of the removed transitions and let $\mathcal{A}=\left\{C \in \mathcal{F}_{v} \backslash\left\{C_{v}\right\} \mid C\right.$ contains $v$. $\}$.

By the choice of $(G, \mathcal{T}), \mathcal{F}_{v}$ is an almost compatible circuit decomposition with respect to $v$.

Construct an auxiliary graph $\mathcal{I}$ with the vertex set $V(\mathcal{I})=\mathcal{F}_{v}$ and two vertices of $\mathcal{I}$ are adjacent to each other if and only if their corresponding circuits of $\mathcal{F}_{v}$ have a non-empty intersection in $G \backslash\{v\}$. Since $G$ is 2 -connected, $\mathcal{I}$ is connected. Let $S=C_{1} \ldots C_{k}$ be a shortest path in $\mathcal{I}$ from $C_{1}=C_{v}$ to $\mathcal{A}\left(C_{k} \in \mathcal{A}\right)$. Obviously, $S$ is a circuit chain of $G$ closed at $v$.

Let $G^{\prime \prime}$ be the subgraph induced by edges of $\cup_{i=1}^{k} E\left(C_{i}\right)$. The transitioned graph $\left(G^{\prime \prime},\left.\mathcal{T}\right|_{G^{\prime \prime}}\right)$ is 2 -connected, so it has no bad cut-vertex. Thus, every circuit $C \in \mathcal{F}_{v} \backslash\left\{C_{1}, \ldots, C_{k}\right\}$ is a removable circuit. This is impossible. Therefore, $\mathcal{F}_{v}=\left\{C_{1}, \ldots, C_{k}\right\}$ is an $\operatorname{ACCCD}(v)$ of $(G, \mathcal{T})$ and $G$ is 4 -regular. 


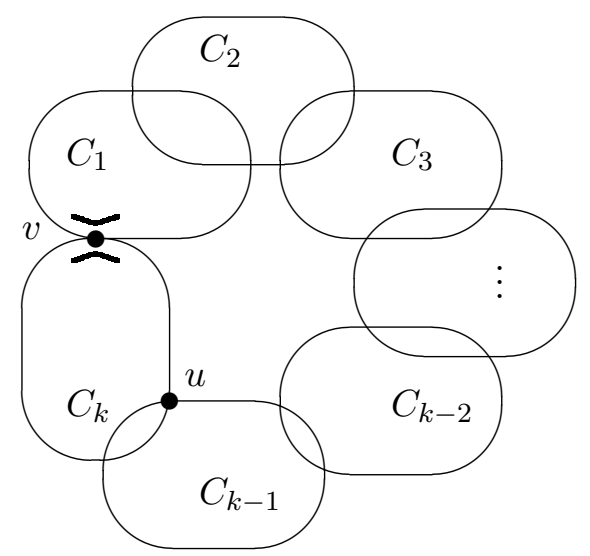

Figure 1.7: $A n \operatorname{ACCCD}(v)$ of $(G, \mathcal{T})$.

Lemma 1.4.3. Any smallest counterexample to Theorem 1.1.1' has no digon of type $\lambda>0$.

Proof. Let $(G, \mathcal{T})$ be a smallest counterexample to Theorem 1.1.1'. Suppose $(G, \mathcal{T})$ has a digon of type $\lambda>0, D$. The smaller graph $\left(G^{\prime}, \mathcal{T}^{\prime}\right)$ obtained from $(G, \mathcal{T})$ by contracting $D$ remains SUD- $K_{5}$-minor-free, because $(G, \mathcal{T})$ has this property. Thus it has a CCD. It is easily seen that every $\mathrm{CCD}$ of $\left(G^{\prime}, \mathcal{T}^{\prime}\right)$ induces a $\mathrm{CCD}$ on $(G, \mathcal{T})$, which is a contradiction.

Lemma 1.4.4. Any smallest counterexample to Theorem 1.1.1' is 4-edge-connected.

Proof. Let $(G, \mathcal{T})$ be a smallest counterexample to Theorem 1.1.1'. Assume that $\left\{e_{1}, e_{2}\right\}$ is a 2-edge-cut of $(G, \mathcal{T})$ and $G_{1}, G_{2}$ are the components of $G \backslash\left\{e_{1}, e_{2}\right\}$. By Lemma 1.4.2, $G$ is 2 -connected, so $e_{1}$ and $e_{2}$ are vertex disjoint. Let $e_{1}=u_{1} u_{2}$ and $e_{2}=v_{1} v_{2}$ where $\left\{u_{i}, v_{i}\right\} \subset V\left(G_{i}\right), i=1,2$.

Let $H_{i}=G / G_{3-i}$ for each $i=1,2$. It is easy to check that $\left(H_{i}, \mathcal{S}_{i}\right), i=1,2$, is SUD- $K_{5-}$ minor-free, $\mathcal{S}_{i}=\left.\mathcal{T}\right|_{G_{i}}$. So there exists a $\operatorname{CCD} \mathcal{C}_{i}$ of $\left(H_{i}, \mathcal{S}_{i}\right)$ and a circuit $C_{i} \in \mathcal{C}_{i}$ covering $u_{i} v_{i}$, $i=1,2$. Let $C=\left(C_{1} \cup C_{2} \cup\left\{u_{1} u_{2}, v_{1} v_{2}\right\}\right) \backslash\left\{u_{1} v_{1}, u_{2} v_{2}\right\}$. Thus, $\mathcal{C}=\left(\mathcal{C}_{1} \cup \mathcal{C}_{2} \cup\{C\}\right) \backslash\left\{C_{1}, C_{2}\right\}$ is a $\mathrm{CCD}$ of $(G, \mathcal{T})$, a contradiction.

Since no eulerian graph has an edge-cut of odd size, $(G, \mathcal{T})$ is 4 -edge-connected.

Lemma 1.4.5. Any smallest counterexample to Theorem 1.1.1' is 3-connected.

Proof. Let $(G, \mathcal{T})$ be a smallest counterexample to Theorem 1.1.1'. By Lemma 1.4.2, $G$ is a 2-connected 4-regular graph. By Lemma 1.4.4, $G \backslash X$ has exactly two components, for every 2 -vertex-cut $X$.

Suppose $\{u, v\}$ is a 2 -vertex-cut of $G$ such that $G_{1}, G_{2}$ are the components of $G \backslash\{u, v\}$. Every edge-cut in an eulerian graph has an even number of edges. It follows that $u, v$ can be 
chosen such that for $i=1,2$, both $u$ and $v$ have the same degrees in $G \backslash V\left(G_{i}\right)$. By Lemma 1.4.4, $u v \notin E(G)$ and $\operatorname{deg}_{G \backslash V\left(G_{i}\right)}(u)=\operatorname{deg}_{G \backslash V\left(G_{i}\right)}(v)=2, i=1,2$. We have two cases (see Figure 1.8).

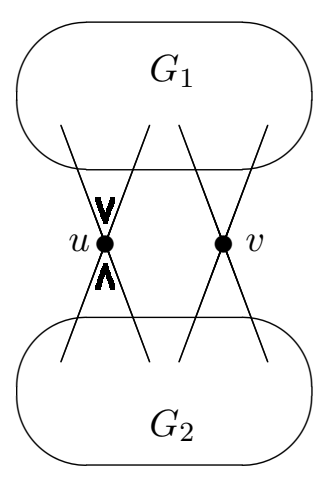

Case 1

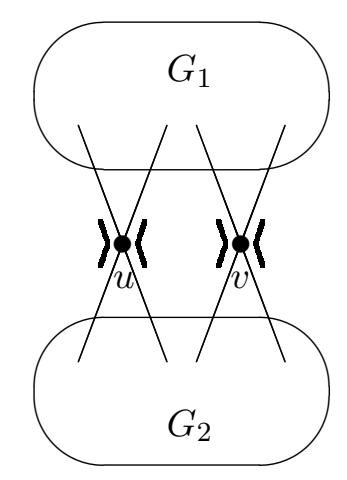

Case 2

Figure 1.8: 2-vertex-cut $\{u, v\}$.

Case 1. $E\left(G \backslash V\left(G_{i}\right)\right) \cap E(u) \in \mathcal{T}(u)$.

In this case, let $\left(G_{i}^{\prime}, \mathcal{T}_{i}^{\prime}\right)$ be a transitioned 4 -regular graph obtained from $(G, \mathcal{T})$ by contracting all edges of $G \backslash V\left(G_{i}\right)$. Then, $\left(G_{i}^{\prime}, \mathcal{T}_{i}^{\prime}\right)$ has no SUD- $K_{5}$-minor. It follows from the minimality of $(G, \mathcal{T})$ that $\left(G_{i}^{\prime}, \mathcal{T}_{i}^{\prime}\right)$ has a CCD. Then by adapting the circuits containing edges of $E(u) \cup E(v)$ in these two CCD's, we may obtain a CCD of $(G, \mathcal{T})$, which is a contradiction.

Case 2. $\left\{u_{1} u, u u_{2}\right\} \in \mathcal{T}(u),\left\{v_{1} v, v v_{2}\right\} \in \mathcal{T}(v)$, where $u_{i}, v_{i}$ are neighbours of $u$ and $v$ in $G_{i}$, $i=1,2$, respectively.

In this case, we set $G_{i}^{\prime}=G \backslash V\left(G_{i+1}\right)$, and define $\mathcal{T}_{i}^{\prime}$ as the set of transitions in $G_{i}^{\prime}$ induced by $\left.\mathcal{T}\right|_{G_{i}^{\prime}}$. Observe that $\left(G_{1}^{\prime}, \mathcal{T}_{1}^{\prime}\right)$ and $\left(G_{2}^{\prime}, \mathcal{T}_{2}^{\prime}\right)$ have no bad cut-vertex; otherwise, the bad cut-vertex and vertex $u$ is a 2 -vertex-cut yielding Case 1 . Therefore, $\left(G_{i}^{\prime}, \mathcal{T}_{i}^{\prime}\right)$ has a CCD, $i=1,2$. The union of these two CCD's is a CCD of $(G, \mathcal{T})$, which is a contradiction.

Lemma 1.4.5 now follows.

Corollary 1.4.6. Any smallest counterexample to Theorem 1.1.1' has no digon.

Proof. Let $(G, \mathcal{T})$ be a smallest counterexample to Theorem 1.1.1'. Suppose $(G, \mathcal{T})$ has a digon, $D$. By Lemma 1.4.3, $D$ is a digon of type 0. Then by Lemma 1.4.5, $G \backslash E(D)$ is 2-connected. Thus, $D$ is a removable circuit, which is a contradiction.

Definition 1.4.2. An even subgraph $H$ of $(G, \mathcal{T})$ is compatible if $|E(H) \cap P| \leq 1$, for every $P \in \mathcal{T}$. An almost compatible 2-even subgraph decomposition $\left\{U_{1}, U_{2}\right\}$ with respect to $v$ is 
a decomposition into two even subgraphs in such a way that both $U_{i}$ 's are compatible at every $w \in V(G) \backslash\{v\}$, and $U_{i}$ is not compatible at $v$ for at least one $i$.

Definition 1.4.3. Let $(G, \mathcal{T})$ be a transitioned 4 -regular graph. Let $v$ be a non-trivial vertex of degree 4 in $(G, \mathcal{T}$ ) and let $\{e, f\} \in \mathcal{T}(v)$. By splitting $v$ (with respect to $\mathcal{T}$ ) we mean that $v$ is split into two degree 2 vertices such that $e$ and $f$ are incident with the same vertex. The split graph of $(G, \mathcal{T})$, denoted by $S P(G, \mathcal{T})$, is the graph obtained from $(G, \mathcal{T})$ by splitting every non-trivial vertex.

The following lemma appeared in $[1,9]$ as part of proofs of some theorems (not as an independent lemma). For the purpose of smoothness of the chapter and possible applications in the future, Lemma 1.4.7 is stated in this chapter as an independent lemma. The proof is also included here for the purpose of not only the consistency of notation and terminology but also for the self-completeness of the chapter.

Lemma 1.4.7. $[1,9]$ Let $(G, \mathcal{T})$ be a smallest counterexample to Theorem 1.1.1'. Then

(1) $S P(G, \mathcal{T})$ has exactly two components;

(2) for each non-trivial vertex $v$, if $x$ and $y$ are the two vertices in $S P(G, \mathcal{T})$ which result by splitting $v$, then they are contained in different components of $\operatorname{SP}(G, \mathcal{T})$;

(3) each component of $S P(G, \mathcal{T})$ is a circuit of odd length.

Proof. Let $(G, \mathcal{T})$ be a smallest counterexample to Theorem 1.1.1'. By Lemma 1.4.2, $G$ is 4 -regular and for every non-trivial vertex $v \in V(G)$, there exists an $\operatorname{ACCCD}(v)$, say $\mathcal{F}_{v}=$ $\left\{C_{1}, \ldots, C_{k}\right\}$.

Let

$$
S_{1}=\cup_{\mu=1}^{\left\lceil\frac{k}{2}\right\rceil} E\left(C_{2 \mu-1}\right) \quad \text { and } \quad S_{2}=\cup_{\mu=1}^{\left\lfloor\frac{k}{2}\right\rfloor} E\left(C_{2 \mu}\right)
$$

Then, $\left\{S_{1}, S_{2}\right\}$ is an almost compatible 2 -even subgraph decomposition with respect to $v$. Note that depending on the parity of $k, v \in V\left(S_{2}\right)$ if and only if $k$ is even. If $k$ is odd then $S_{2}$ is a set of compatible circuits.

Next, to establish the validity of the Lemma we prove a sequence of claims.

Claim 1.4.1. For every almost compatible 2 - even subgraph decomposition $\left\{U_{1}, U_{2}\right\}$ with respect to $v$, for every vertex $w \neq v, \operatorname{deg}_{U_{i}}(w)=2, i=1,2$.

Assume that $\left\{U_{1}, U_{2}\right\}$ is an almost compatible 2-even subgraph decomposition with respect to $v$ and that there exists a vertex $w \neq v, \operatorname{deg}_{U_{1}}(w)=4$. By Definition 1.4.2, a non-trivial vertex of $G$ other than $v$ cannot be of degree 4 in $U_{i}, i=1,2$. Thus, $w$ is a trivial vertex and $E(w) \subseteq E\left(U_{1}\right)$. 
Let $\mathcal{F}_{i}$ be a circuit decomposition of $U_{i}$ for each $i=1,2$. The union $\mathcal{F}_{1} \cup \mathcal{F}_{2}$ forms an almost compatible circuit decomposition with respect to $v$, by the choice of $(G, \mathcal{T})$. By Lemma 1.4.2, every almost CCD with respect to a non-trivial vertex is a circuit chain, hence $\mathcal{F}_{1} \cup \mathcal{F}_{2}$ is a circuit chain $\left\{D_{1}, \ldots, D_{r}\right\}$. Since $G\left[U_{1}\right]$ has a vertex of degree 4 , it follows that $r \geq 3$. By Lemma 1.4.1, we have $V\left(D_{1}\right) \cap V\left(D_{r}\right)=\{v\}$. Let $w \in V\left(D_{j}\right) \cap V\left(D_{j+1}\right)$. Note that $D_{j}$ and $D_{j+1}$ are edge-disjoint and both are subsets of $U_{1}$. So, every vertex of the induced subgraph $G\left[D_{j} \cup D_{j+1}\right]$ is of degree 2 or 4 . If $w$ is the only vertex of $V\left(D_{j}\right) \cap V\left(D_{j+1}\right)$, then $\{v, w\}$ is a 2 -vertex-cut of $G$ (since $G$ has no digon by Corollary 1.4.6). This contradicts Lemma 1.4.5.

Thus the induced subgraph $G\left[D_{j} \cup D_{j+1}\right]$ is 2-connected. Let $u_{j} \in V\left(D_{j}\right) \cap V\left(D_{j-1}\right)$ (or $u_{j}=v$ if $j=1$ ), and let $u_{j+1} \in V\left(D_{j+1}\right) \cap V\left(D_{j+2}\right)$ (or $u_{j+1}=v$ if $j+1=r$ ). Let $D \subset G\left[D_{j} \cup D_{j+1}\right]$ be a circuit containing the vertices $u_{j}$ and $u_{j+1}$. Then $G\left[D_{j} \cup D_{j+1}\right] \backslash D$ is a removable even subgraph of $(G, \mathcal{T})$. This is a contradiction. Thus, $\operatorname{deg}_{U_{i}}(w)=2$, for every $w \neq v, i=1,2$, and thus Claim 1.4.1 is true.

The following claim is obvious.

Claim 1.4.2. For each circuit $C$ of $S P(G, \mathcal{T}),\left\{S_{1} \Delta C, S_{2} \Delta C\right\}$ is also an almost compatible 2 -even subgraph decomposition with respect to $v$.

Claim 1.4.3. For each trivial vertex $w$ with $\left\{e^{\prime}, e^{\prime \prime}\right\}=E(w) \cap S_{1}$, no circuit of $S P(G, \mathcal{T})$ contains both edges $e^{\prime}$ and $e^{\prime \prime}$.

Suppose that $C$ is a circuit of $S P(G, \mathcal{T})$ containing both edges $e^{\prime}$ and $e^{\prime \prime}$. By Claim 1.4.2, $\left\{S_{1} \Delta C, S_{2} \Delta C\right\}$ is also an almost compatible 2-even subgraph decomposition with respect to $v$. Note that $\operatorname{deg}_{S_{2} \Delta C}(w)=4$. This contradicts Claim 1.4.1. Thus Claim 1.4.3 now follows.

Therefore, by Claim 1.4.3, we have the following immediate conclusions about $S P(G, \mathcal{T})$. Let $w$ be a trivial vertex of $(G, \mathcal{T})$.

Claim 1.4.4. For each pair $\left\{e^{\prime}, e^{\prime \prime}\right\}=E(w) \cap S_{i}(i=1,2)$, the edges $e^{\prime}$ and $e^{\prime \prime}$ must be in different blocks of $\operatorname{SP}(G, \mathcal{T})$.

From Claim 1.4.4, we conclude

Claim 1.4.5. The trivial vertex $w$ must be a cut-vertex of some component of $S P(G, \mathcal{T})$.

This also implies

Claim 1.4.6. The circuit decomposition of $S P(G, \mathcal{T})$ is unique.

Notation. Let $R_{1}, \ldots, R_{h}$ be the components of the split graph $S P(G, \mathcal{T})$, and let $\left\{X_{1}, \ldots, X_{t}\right\}$ be the unique circuit decomposition of $S P(G, \mathcal{T})$, which is also the block decomposition of $S P(G, \mathcal{T})$. 
Claim 1.4.7. Let $x$ and $y$ be the two vertices in $S P(G, \mathcal{T})$ which result from by splitting $v$. Then $x$ and $y$ are contained in different components of $S P(G, \mathcal{T})$.

Proceeding by contradiction, suppose that $x$ and $y$ are contained in the same component $R_{1}$, of $S P(G, \mathcal{T})$. Let $P$ be a path of $R_{1}$ joining $x$ and $y$. Let $C$ be the even subgraph induced by $E(P)$ in $G$. Note that $C$ is not compatible in its vertices except at $v . S_{1}$ and $S_{2}$ are compatible at every vertex $u \neq v$, and $S_{1}$ is not compatible at vertex $v$. Therefore, $\left\{S_{1} \Delta C, S_{2} \Delta C\right\}$ is a compatible 2-even subgraph decomposition which is a contradiction to the choice of $G$ and thus proves the claim.

By Claim 1.4.7 assume without loss of generality that $x \in X_{1}$ and $y \in X_{2}$ where $X_{j}$ is a block of $R_{j}, j=1,2$.

Claim 1.4.8. The circuits $X_{1}$ and $X_{2}$ are of odd lengths, while all other $X_{i}(i>2)$ are of even lengths.

Color the edges of $S_{1}$ with blue, and the edges of $S_{2}$ with red. By Claim 1.4.4, each circuit $X_{i}$ is of even length if $i \neq 1,2$ since its edges are alternately colored with red and blue, while $X_{1}$ and $X_{2}$ are of odd length since each of $x, y$ is incident with two edges of the same color. Claim 1.4.8 now follows.

The following is the final claim and concludes the proof of the lemma.

Claim 1.4.9. $h=t=2$. That is, the split graph $S P(G, \mathcal{T})$ has precisely components $R_{1}=X_{1}$ and $R_{2}=X_{2}$ each of which is a circuit of odd length.

Since the non-trivial vertex $v$ was selected arbitrarily, all conclusions we have had above can be applied to every non-trivial vertex; that is, for every non-trivial vertex $v$ and the vertices $x$ and $y$ resulting by splitting $v$, it follows that $x \in X_{1}$ and $y \in X_{2}$.

If $R_{1}$ has more than one block, then $R_{1}$ must have a block $Q_{3}$ other than $X_{1}$ that contains precisely one cut-vertex $z$ of $R_{1}$ (note that $Q_{3}$ corresponds to a leaf in the block-cut-vertex graph of $R_{1}$ ). By Claims 1.4.7 and 1.4.8, every vertex of $Q_{3}$ is trivial. So by Claim 1.4.5, every vertex of $Q_{3}$ is a cut-vertex of $S P(G, \mathcal{T})$. This contradicts the supposed existence of $Q_{3}$.

Furthermore, no edge of $R_{i}$ with $i>2$ is incident with a non-trivial vertex. By the definition of $S P(G, \mathcal{T})$, each $R_{i}$ with $i>2$ also corresponds to a component of $G$ whose vertices are all trivial. This contradicts $G$ being connected.

Therefore, $S P(G, \mathcal{T})$ consists of two vertex disjoint circuits of odd length $X_{1}=R_{1}$ and $X_{2}=R_{2}$. Lemma 1.4.7 now follows.

Since in the proof of Lemma 1.4.7, it is shown that any smallest counterexample to Theorem 1.1.1' has no trivial vertex, we have the following corollary.

Corollary 1.4.8. Any smallest counterexample to Theorem 1.1.1' is a fully transitioned graph. 
Lemma 1.4.9. [9] Let $(G, \mathcal{T})$ be a smallest counterexample to Theorem 1.1.1' and let $\mathcal{F}_{v}=$ $\left\{C_{1}, \ldots, C_{k}\right\}$ be an ACCCD of $(G, \mathcal{T})$ with respect to a non-trivial vertex $v$ with $k=\left|\mathcal{F}_{v}\right|$ maximum. Then $k \geq 3$.

Proof. Since $v$ is of degree $4, k>1$ where $\mathcal{F}_{v}=\left\{C_{1}, \ldots, C_{k}\right\}$. Assume that $k=2$. Let $R_{1}$ and $R_{2}$ be the components of $S P(G, \mathcal{T}$ ) (see Lemma 1.4.7 (1)). By Lemma 1.4 .7 and Definition 1.4.3, without loss of generality, let $E(v) \cap E\left(C_{1}\right) \subseteq E\left(R_{1}\right)$ and $E(v) \cap E\left(C_{2}\right) \subseteq E\left(R_{2}\right)$. Consider $\left\{C_{1} \Delta R_{1}, C_{2} \Delta R_{1}\right\}$. It is easy to check that $\left\{C_{1} \Delta R_{1}, C_{2} \Delta R_{1}\right\}$ is an almost compatible decomposition into even subgraphs of $(G, \mathcal{T})$ with respect to $v$. Note that $E(v) \subseteq E\left(C_{2} \Delta R_{1}\right)$. Therefore, the maximum degree of $C_{2} \Delta R_{1}$ is four and hence any of its circuit decomposition consists of at least two circuits. Since $S P(G, \mathcal{T})$ has two components and $G$ is 2-connected, $(G, \mathcal{T})$ has at least a second non-trivial vertex $u \neq v$. Because $C_{1}$ is compatible in $u, C_{1} \Delta R_{1}$ is not empty. Therefore, the union of circuit decompositions of $C_{1} \Delta R_{1}$ and $C_{2} \Delta R_{1}$ has at least three elements. This contradicts the maximality of $\left|\mathcal{F}_{v}\right|$.

\subsubsection{Cornered triangle extension property: key lemmas for the determina- tion of UD- $K_{5}$}

There are few results in graph theory that tell us the existence of the Petersen-minor (for example, $[8,26]$, etc). The main lemmas in this section provide a new approach to identify the precise structure of a transitioned UD- $K_{5}$ (their corresponding versions for the faithful circuit covering problem identify the Petersen graph). These lemmas are applied in the final steps of the proofs of Theorems 1.1.1' and 1.1.2.

Definition 1.4.4. Let $C_{0}=x y_{1} y_{2} x$ be a non-compatible circuit of length 3 .

(1) The corner of $C_{0}$ is a given inner vertex, say $x$, of the triangle. If $y_{j}$ is a compatible vertex of $C_{0}$, then the opposite edge $x y_{i}$ is called a leg of $C_{0}(i \neq j)$.

(2) For $\mu=1,2$, a triangle $C_{0}$ with the corner $x$ is called $\mu$-legged if $E(x) \cap E\left(C_{0}\right)$ contains at least $\mu$ legs.

(3) Let $C_{0}=x y_{1} y_{2} x$ be a triangle with the corner $x$. Given $x y_{i}$ a leg of $C_{0}$, an extension of $C_{0}$ along the leg $x y_{i}$ is another triangle $C_{i}=w_{i} x y_{i} w_{i}$ with the corner $w_{i}$ where $w_{i} \notin V\left(C_{0}\right)$ (note that $y_{i} w_{i}$ is a leg of $C_{i}$ ).

(4) A $\mu$-legged triangle $C_{0}=x y_{1} y_{2} x$ with the corner $x$ is $\mu$-extendable if every leg $x y_{i}$ has an extension which is also $\mu$-legged ( a $\mu$-legged extension; see Figure 1.9).

Definition 1.4.5. For a given integer $\mu \in\{1,2\}$, a graph $G$ has the the $\mu$-legged-triangleextension property (abbreviated as $\mu$-LTEP) if $G$ contains some $\mu$-legged triangle and each of them is $\mu$-extendable (see Definition 1.4.4(4)). 

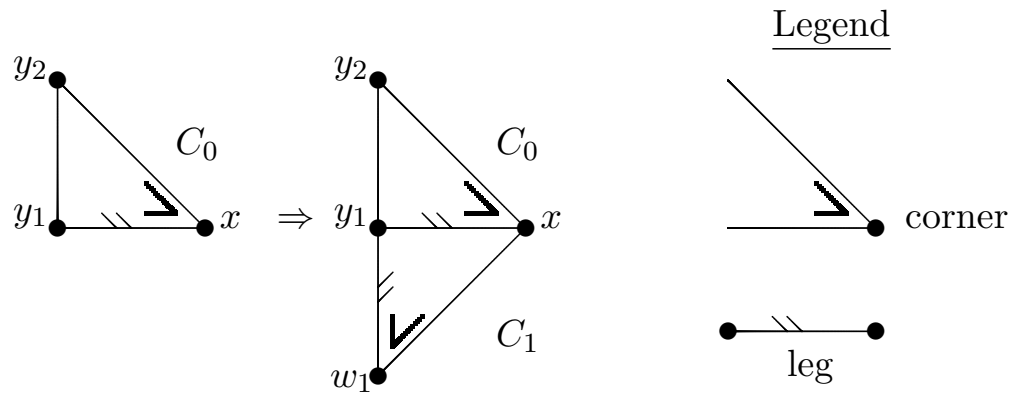

Figure 1.9: A cornered triangle $C_{0}=x y_{1} y_{2} x$, and its extension $C_{1}=w_{1} x y_{1} w_{1}$

The $\mu$-legged-triangle-extension property is an inductive hypothesis, which means one can get an extension sequence of $\mu$-legged triangles starting from a fix $\mu$-legged triangle.

The following two lemmas play an important role in the proofs of the main theorems. These lemmas identify the structure of the UD- $K_{5}$ based on the extension property.

In the proofs of the main theorems, the 1-LTEP or 2-LTEP will be verified for smallest counterexamples to the theorems. We wish to point out that although Lemma 1.4.10 and Lemma 1.4.11 look very similar, neither of them is an immediate corollary of the other.

Lemma 1.4.10. Let $(G, \mathcal{T})$ be a 4-regular, fully transitioned, simple graph. If $(G, \mathcal{T})$ has the 2-LTEP, then it is exactly the UD- $K_{5}$.

Proof. By the 2-LTEP, there exists a 2-legged triangle in $(G, \mathcal{T})$, say $S_{0}=v v_{1} v_{2} v$, with corner $v$ and two legs $v v_{1}$ and $v v_{2}$. Since $S_{0}$ has the 2-LTEP, each leg $v v_{i}(i=1,2)$, has a 2-legged extension $S_{i}=v_{i+2} v v_{i} v_{i+2}$ which is also a 2-legged triangle with the corner $v_{i+2}$.

Since $G$ is simple, it can be seen that $v_{3} \neq v_{4}$, for otherwise, by looking at the transitions contained in $E\left(v_{3}\right)$, the edge $v v_{3}$ would be contained in two distinct transitions $\left\{v_{3} v, v_{3} v_{1}\right\}$ and $\left\{v_{3} v, v_{3} v_{2}\right\}$ (see Figure 1.10-(ii)).

Since $S_{i}$ has the 2-LTEP $(i=1,2)$, each leg $v v_{i+2}$ has a 2-legged extension $S_{i+2}=w_{i} v v_{i+2} w_{i}$. Since $G$ is 4-regular, $w_{1} \in\left\{v_{2}, v_{4}\right\}$ and $w_{2} \in\left\{v_{1}, v_{3}\right\}$. Since the transition $\left\{v_{4} v, v_{4} v_{2}\right\} \in \mathcal{T}\left(v_{4}\right)$ and $w_{1}$ is an inner vertex of $S_{3}$, we have that $w_{1} \neq v_{4}$. Hence, $w_{1}=v_{2}$. Symmetrically, $w_{2}=v_{1}$.

Since $S_{1}$ has the 2-LTEP, the leg $v_{1} v_{3}$, has a 2-legged extension $S_{5}=w_{3} v_{1} v_{3} w_{3}$ with corner $w_{3}$. By the 4-regularity of $G, w_{3} \in\left\{v, v_{2}, v_{4}\right\}$. Since $w_{3}$ is an inner vertex of $S_{5}$, one has $w_{3}=v_{4}$ by looking at the transitions at $v$ and $v_{2}$. Thus, $\left\{v_{4} v_{1}, v_{4} v_{3}\right\} \in \mathcal{T}\left(v_{4}\right)$, and $\left\{v_{3} v_{2}, v_{3} v_{4}\right\} \in \mathcal{T}\left(v_{3}\right)$ (see Figure 1.10-(iii)).

It is now easy to check that $(G, \mathcal{T})$ is exactly the UD- $K_{5}$. 


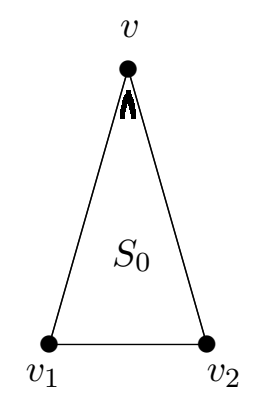

(i)

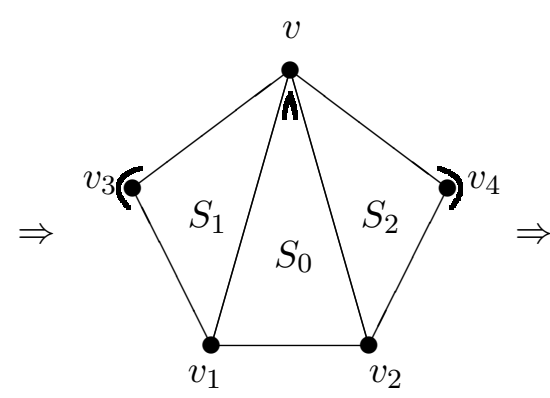

(ii)

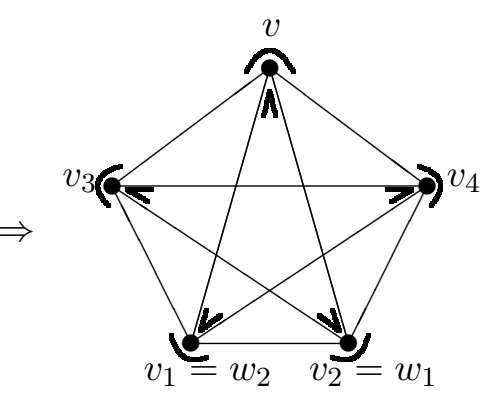

(iii)

Figure 1.10: Proof of Lemma 1.4.10

Lemma 1.4.11. Let $(G, \mathcal{T})$ be a 4-regular, 4-edge-connected, fully transitioned, simple graph. If $(G, \mathcal{T})$ has the 1-LTEP, then either it is the UD-K $K_{5}$ or it has a CCD of size 3.

Proof. Let $S_{1}=v_{0} v_{1} v_{2} v_{0}$ be a 1-legged triangle with the corner $v_{2}$ and a leg $v_{0} v_{2}$. By using the 1-LTEP of $S_{1}$ at the leg $v_{0} v_{2}$, we have a new vertex $v_{3}$ such that $S_{2}=v_{0} v_{2} v_{3} v_{0}$ is a 1-legged triangle with the corner $v_{3}$ and a leg $v_{0} v_{3}$.

By using the 1-LTEP of $S_{2}$ at the leg $v_{0} v_{3}$, there is a 1-legged triangle $S_{3}=v_{0} v_{3} w_{0} v_{0}$ with the corner $w_{0}$ and a leg $v_{0} w_{0}$. Since $S_{3} \neq S_{2}$ and $G$ is simple, there are two possibilities for $w_{0}$ : $w_{0}=v_{1}$ or $w_{0} \notin\left\{v_{0}, \ldots, v_{3}\right\}$.

Case A: $w_{0}=v_{1}$ (see Figure 1.11).
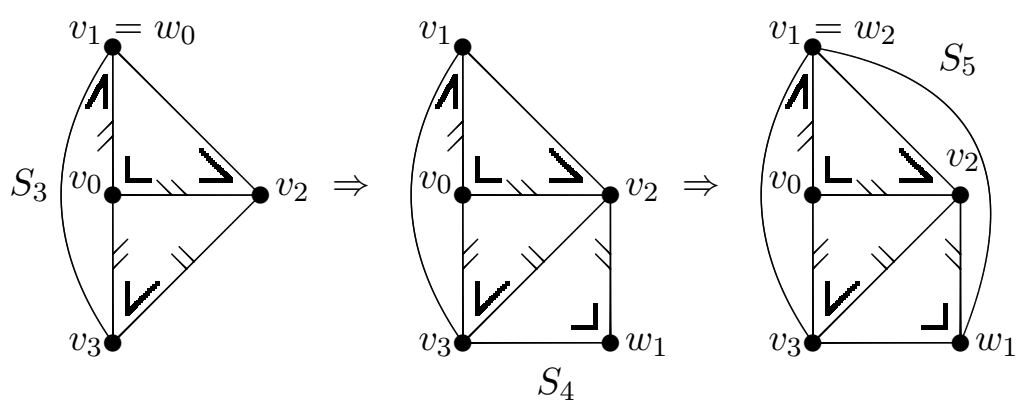

Figure 1.11: Case A $\left(w_{0}=v_{1}\right)$

We will show that this case cannot happen.

Since $(G, \mathcal{T})$ is fully transitioned, there exists a transition of $v_{0}$ contained in the edge set $\left\{v_{0} v_{1}, v_{0} v_{2}, v_{0} v_{3}\right\}$. By rotational symmetry, we may assume that $\left\{v_{0} v_{1}, v_{0} v_{2}\right\} \in \mathcal{T}\left(v_{0}\right)$. Thus $v_{2} v_{3}$ is another leg of the 2-legged triangle $S_{2}$. By using the 1-LTEP of $S_{2}$ at the leg $v_{2} v_{3}$, there exists a 1-legged triangle $S_{4}=v_{2} v_{3} w_{1} v_{2}$ with the corner $w_{1}$ and a leg $v_{2} w_{1}$. It is obvious that $w_{1} \notin\left\{v_{0}, v_{2}, v_{3}\right\}$. If $w_{1}=v_{1}$, then the edge $v_{1} v_{3}$ will be contained two distinct transitions, which is impossible. 
By using the 1-LTEP of $S_{4}$ at the leg $v_{2} w_{1}$, there exists a 1-legged triangle $S_{5}=v_{2} w_{1} w_{2} v_{2}$ with the corner $w_{2}$ and a leg $v_{2} w_{2}$. Since $G$ is 4 -regular and simple, $w_{2} \in\left\{v_{0}, v_{1}\right\}$. If the corner $w_{2}=v_{0}$, then $\left\{w_{2} w_{1}, w_{2} v_{2}\right\}=\left\{v_{0} w_{1}, v_{0} v_{2}\right\} \in \mathcal{T}\left(v_{0}\right)$. But the edge $v_{0} v_{2}$ is already contained in another transition $\left\{v_{0} v_{1}, v_{0} v_{2}\right\}$. This is a contraction, and therefore, $w_{2}=v_{1}$.

Let $e^{\prime} \in E\left(v_{0}\right)-\left\{v_{0} v_{1}, v_{0} v_{2}, v_{0} v_{3}\right\}$ and $e^{\prime \prime} \in E\left(w_{1}\right)-\left\{w_{1} v_{1}, w_{1} v_{2}, w_{1} v_{3}\right\}$. Since $G$ is 4-regular and 4-edge-connected, we have that $e^{\prime}=e^{\prime \prime}$ for otherwise $\left\{e^{\prime}, e^{\prime \prime}\right\}$ is a 2-edge-cut of $G$. That is, $e^{\prime}=e^{\prime \prime}=w_{1} v_{0}$, and $V(G)=\left\{v_{0}, v_{1}, v_{2}, v_{3}, w_{1}\right\}$.

Consider the 2-legged triangle $v_{0} w_{1} v_{3} v_{0}$ with corner $v_{0}$. By using the 1-LTEP at the leg $v_{0} w_{1}$, there exists a 1-legged triangle $v_{0} w_{1} w_{3} v_{0}$ with the corner $w_{3}$. By the 4-regularity of $G$, one must have $w_{3}=v_{1}$ or $w_{3}=v_{2}$. However, none of them can happen as can be seen by checking the transitions around $v_{1}$ and $v_{2}$.

Case B: $w_{0} \notin\left\{v_{0}, \ldots, v_{3}\right\}$; denote $w_{0}=v_{4}$ (see Figure 1.12).

By using the 1-LTEP of $S_{3}$ at the leg $v_{0} v_{4}$, there exists a 1-legged triangle $S_{6}=v_{0} v_{4} w_{3} v_{0}$ with the corner $w_{3}$ and a leg $v_{0} w_{3}$. Since $G$ is 4 -regular and simple, $w_{3} \in\left\{v_{1}, v_{2}\right\}$. If $w_{3}=v_{2}$, then the edge $v_{0} v_{2}$ is contained in the two transitions $\left\{v_{2} v_{0}, v_{2} v_{1}\right\}$ and $\left\{v_{2} v_{0}, v_{2} v_{4}\right\}$ of $v_{2}$. This is a contradiction. Therefore, $w_{3}=v_{1}$.

Note there is no information yet about the transitions around the vertex $v_{0}$. By symmetry, there are two cases for further analysis:

$$
\left\{v_{0} v_{1}, v_{0} v_{2}\right\} \in \mathcal{T}\left(v_{0}\right) \text { or }\left\{v_{0} v_{1}, v_{0} v_{3}\right\} \in \mathcal{T}\left(v_{0}\right)
$$

In either case, we can assume that $v_{0}$ is compatible in the triangle $S_{2}=v_{0} v_{2} v_{3} v_{0}$. That is, the edge $v_{2} v_{3}$ is another leg of the triangle $S_{2}$. By using the 1-LTEP of $S_{2}$ at the leg $v_{2} v_{3}$, we have an extension $S_{7}=v_{2} v_{3} w_{4} v_{2}$ with the corner $w_{4}$ and a leg $v_{2} w_{4}$. Proceeding similarly to the above, by looking at the transitions around $v_{4}$, we have that $w_{4} \neq v_{4}$. Hence, there are two possibilities for $w_{4}: w_{4} \notin\left\{v_{0}, \ldots, v_{4}\right\}$ or $w_{4}=v_{1}$ (see Figure 1.12).

Subcase B-1. $w_{4} \notin\left\{v_{0}, \ldots, v_{4}\right\}$; denote $w_{4}=v_{5}$ (see Figure 1.13).

For this subcase, we will find a CCD of size 3. By using the 1-LTEP of $S_{7}$ at the leg $v_{2} v_{5}=v_{2} w_{4}$, there exists an extension $v_{2} v_{5} w_{5} v_{2}$ with the corner $w_{5}$ and a leg $v_{2} w_{5}$. Since $G$ is 4 -regular and simple and $w_{5} \in\left[N\left(v_{2}\right) \cap N\left(v_{5}\right)\right]-V\left(S_{7}\right)$, we have $w_{5}=v_{1}$ (see Figure 1.13). Arguing similarly as above, we then get $v_{4} v_{5} \in E(G)$ by the 4-edge connectivity and 4-regularity. Therefore $V(G)=\left\{v_{0}, \ldots, v_{5}\right\}$.

By (1.1), if $\left\{v_{0} v_{1}, v_{0} v_{3}\right\} \in \mathcal{T}\left(v_{0}\right)$, then consider the 2-legged triangle $S_{1}=v_{2} v_{1} v_{0} v_{2}$ with the corner $v_{2}$. The leg $v_{1} v_{2}$ cannot be extended by checking at the transitions around $v_{5}$ and the neighborhood of $v_{3}, v_{4}$. This is a contradiction. 
So, by (1.1), we must have $\left\{v_{0} v_{1}, v_{0} v_{2}\right\} \in \mathcal{T}\left(v_{0}\right)$, and thus the set

$$
\left\{v_{1} v_{2} v_{3} v_{4} v_{1}, v_{0} v_{1} v_{5} v_{3} v_{0}, v_{0} v_{2} v_{5} v_{4} v_{0}\right\}
$$

is a $\mathrm{CCD}$ of $(G, \mathcal{T})$ of size 3 .
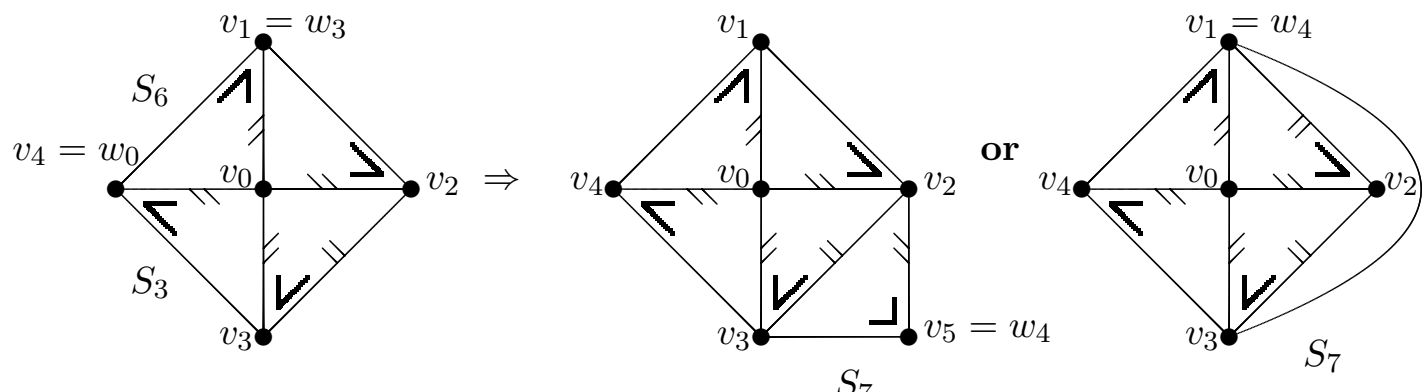

Figure 1.12: Case B $\left(w_{0}=v_{4}\right): S_{7}=v_{2} v_{3} w_{4} v_{2}$ and subcase B-1 $\left(w_{4}=v_{5}\right)$, subcase B-2 $\left(w_{4}=v_{1}\right)$
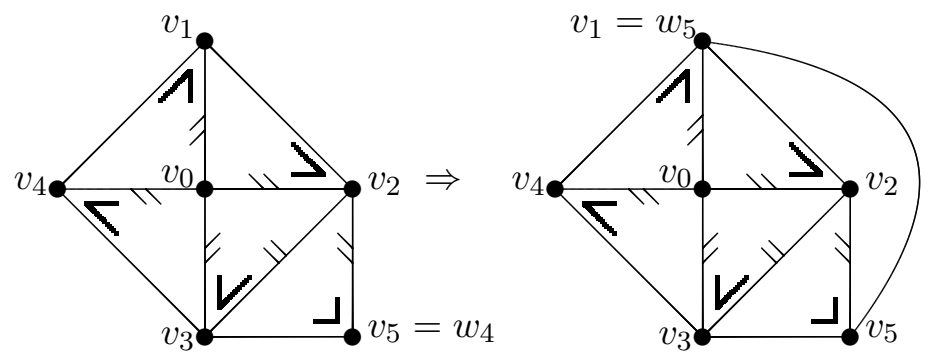

Figure 1.13: Subcase B-1 $\left(w_{4}=v_{5}\right)$.

Subcase B-2. $w_{4}=v_{1}$ (see Figure 1.14).

It is obvious that $v_{2} v_{4} \in E(G)$ by the 4-edge connectivity and 4-regularity of $G$ (see Figure 1.14). By (1.1), we may first assume that $\left\{v_{0} v_{1}, v_{0} v_{2}\right\} \in \mathcal{T}\left(v_{0}\right)$. Then consider the 2-legged triangle $v_{4} v_{2} v_{1} v_{4}$ with the corner $v_{4}$. The leg $v_{2} v_{4}$ cannot be extended by checking at the transitions around $v_{0}$ and $v_{3}$. This is a contradiction.

So, by $(1.1)$, we must have $\left\{v_{0} v_{1}, v_{0} v_{3}\right\} \in \mathcal{T}\left(v_{0}\right)$. It is easy to check that $(G, \mathcal{T})$ is the UD- $K_{5}$ (see Figure 1.14).

\subsection{Simultaneous proof of Theorems 1.1 .1 ' and 1.1.2}

Suppose at least one of these two theorems is false. Let $(G, \mathcal{T})$ be a counterexample to either Theorem 1.1.1' or Theorem 1.1.2 with $|E(G)|$ being as small as possible. Therefore, every admissible transitioned 4 -regular graph without $\operatorname{SUD}-K_{5}$-minor and smaller than $(G, \mathcal{T})$ has 


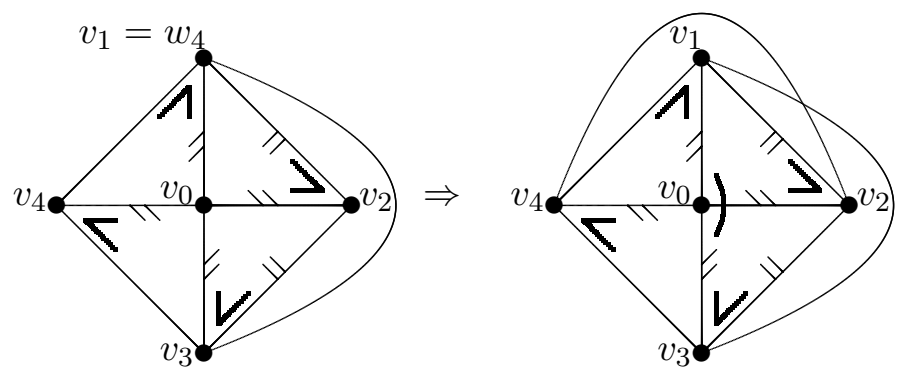

Figure 1.14: Subcase B-2 $\left(v_{1}=w_{4}\right):(G, \mathcal{T})$ is the UD- $K_{5}$.

a $\mathrm{CCD}$; and for every Hamilton transitioned graph $(H, \mathcal{S})$ smaller than $(G, \mathcal{T})$, if $(H, \mathcal{S})$ is SUD- $K_{5}$-minor-free, then $(H, \mathcal{S}) \in\langle 2 L\rangle$.

For our considerations we introduce an extra definition.

Definition 1.5.1. Let $G^{\prime}$ be a graph obtained from $G$ by some operations. A digon $D^{\prime}$ of $G^{\prime}$ is virtual if its corresponding subgraph $D$ in $G$ is a circuit of length $>2$ such that at least one edge of $D^{\prime}$ corresponds to a path of length $>1$ in $D$; otherwise we speak of $D^{\prime}$ as a real digon.

Now we consider two cases with respect to the assumed counterexample.

Case I. $(G, \mathcal{T})$ is a counterexample to Theorem 1.1.1'.

Case II. $(G, \mathcal{T})$ is a counterexample to Theorem 1.1.2.

Actually, both Case I and Case II contain two sub-cases: For Case I, either Theorem 1.1.1' is false with the smallest counterexample $(G, \mathcal{T})$ while Theorem 1.1.2 is true, or both of the two Theorems are false, the smallest counterexample of Theorem 1.1.1', denoted by $(G, \mathcal{T})$, has smaller or equal size to the smallest counterexample of Theorem 1.1.2. Similarly for Case II.

\subsubsection{Case I. $(G, \mathcal{T})$ is a counterexample to Theorem 1.1.1'.}

The goal of our first step is to show that $(G, \mathcal{T})$ has a kind of extension property for a type of cornered triangle, which is to be proved in Lemma 1.5.3.

Definition 1.5.2. A circuit $C=v_{1} v_{2} \ldots v_{k} v_{1}$ is called an almost removable circuit with respect to $v_{1}\left(\operatorname{ARC}\left(v_{1}\right)\right.$, for short $)$ if it is compatible at every vertex except $v_{1}$ such that $(G \backslash$ $\left.E(C),\left.\mathcal{T}\right|_{G \backslash E(C)}\right)$ has no bad cut-vertex.

Note that, for an almost removable circuit $C_{v_{1}}$ with respect to $v_{1}$, if $d\left(v_{1}\right)=4$ and $v_{1}$ is incident with two transitions, say $P_{1}$ and $P_{2}$, then $P_{1}$ is contained in $C_{v_{1}}$ and $P_{2}$ remains in $G \backslash E\left(C_{v_{1}}\right)$. If this case happens, the remaining transition $P_{2}$ is removed from $\left.T\right|_{G \backslash E\left(C_{v_{1}}\right)}$ by Definition 1.2.7-(3). 
Lemma 1.5.1. Let $(G, \mathcal{T})$ be a smallest counterexample to Theorem 1.1.1', and let $C_{v_{1}}$ be a circuit of $G$ containing $v_{1}$. Then $C_{v_{1}}$ is an $\operatorname{ARC}\left(v_{1}\right)$ if and only if there exists an $\operatorname{ACCCD}\left(v_{1}\right)$ $\mathcal{F}_{v_{1}}$ containing $C_{v_{1}}$.

Proof. Sufficiency is trivially true. Let $C_{v_{1}}$ be an $\operatorname{ARC}\left(v_{1}\right)$. Since $(G, \mathcal{T})$ is a smallest counterexample to Theorem 1.1.1', the transitioned graph $\left(G \backslash E\left(C_{v_{1}}\right),\left.\mathcal{T}\right|_{G \backslash E\left(C_{v_{1}}\right)}\right)$ has a CCD, say $\mathcal{C}_{1}$. Note that $\mathcal{C}_{1} \cup\left\{C_{v_{1}}\right\}$ is an $\operatorname{ACCCD}\left(v_{1}\right)$ because of Lemma 1.4.2.

Lemma 1.5.2. Let $(G, \mathcal{T})$ be a smallest counterexample to Theorem 1.1.1', and let $C_{v_{1}}$ be a triangle of $G$ containing $v_{1}$. If $C_{v_{1}}$ is compatible at every vertex except $v_{1}$, then $C_{v_{1}}$ is an $\operatorname{ARC}\left(v_{1}\right)$.

Proof. Let $C_{v_{1}}=v_{1} v_{2} v_{3} v_{1}$ be compatible at every vertex except $v_{1}$. By Definition 1.5.2, we need to show $\left(G \backslash E\left(C_{v_{1}}\right),\left.\mathcal{T}\right|_{G \backslash E\left(C_{v_{1}}\right)}\right)$ has no bad cut-vertex. Assume there exists a cut-vertex $x \neq v_{1}$ in $G$ such that $G$ has two blocks $Q_{1}$ and $Q_{2}$ incident with $x$ and $Q_{1} \cap E(x) \in \mathcal{T}(x)$. If $V\left(Q_{1}\right) \cap V\left(C_{v_{1}}\right)=\left\{v_{2}\right\}$, then $\left\{x, v_{2}\right\}$ is a 2-vertex-cut. If $V\left(Q_{1}\right) \cap V\left(C_{v_{1}}\right)=\left\{v_{1}, v_{2}\right\}$, then $\left\{x, v_{3}\right\}$ is a $2-$ vertex-cut. In both cases we obtain a contradiction to Lemma 1.4.5.

Lemma 1.5.3. Let $(G, \mathcal{T})$ be a smallest counterexample to Theorem 1.1.1'. Then $(G, \mathcal{T})$ has the following properties.

(i) $\operatorname{ARC}(v)$ exists for every vertex $v$;

(ii) a shortest $\mathrm{ARC}$ is of length 3 , and

(iii) for every $\operatorname{ARC}\left(v_{1}\right)=v_{1} v_{2} v_{3} v_{1}$ and for the edge $v_{1} v_{2}$, there exists an $\operatorname{ARC}(w)=w v_{1} v_{2} w$, $w \neq v_{3}$.

Proof. By Lemma 1.4.2, for every vertex $v \in V(G)$, there exists an $\operatorname{ACCCD}(v)$ (see Corollary 1.4.8), and, for every $v \in V(G)$, by Lemma 1.5.1, $(G, \mathcal{T})$ contains an $\operatorname{ARC}(v)$.

Choose $\operatorname{ACR}(v)$ with the smallest length among all ARC's in $(G, \mathcal{T})$ and choose $\operatorname{ACCCD}(v)$, $\mathcal{F}_{v}=\left\{C_{1}, \ldots, C_{k}\right\}$ with maximum length involving this shortest $\operatorname{ACR}(v), C_{k}$ say (see the left side of Figure 1.15).

Let $\left(G^{\prime}, \mathcal{T}^{\prime}\right)$ be obtained from $(G, \mathcal{T})$ by deleting all edges of $C_{k}$ except $u v$ where $u$ is a neighbour of $v$ on $C_{k}$, contracting $u v$ to a new vertex $v^{*}$ and suppressing vertices of degree two.

For every $C^{\prime} \in G^{\prime}$, assume that $C$ is the subgraph of $(G, \mathcal{T})$ induced by $E\left(C^{\prime}\right)$ and vice versa.

Clearly, $\left(G^{\prime}, \mathcal{T}^{\prime}\right)$ has no SUD- $K_{5}$-minor (see the right side of Figure 1.15), and because of the choice of $(G, \mathcal{T})$, we may consider $\mathcal{F}^{\prime}$ to be a CCD of $\left(G^{\prime}, \mathcal{T}^{\prime}\right)$. There exist two circuits $H_{1}^{\prime}$ and $H_{2}^{\prime}$ of $\mathcal{F}$ each of which contains the new vertex $v^{*}$. 


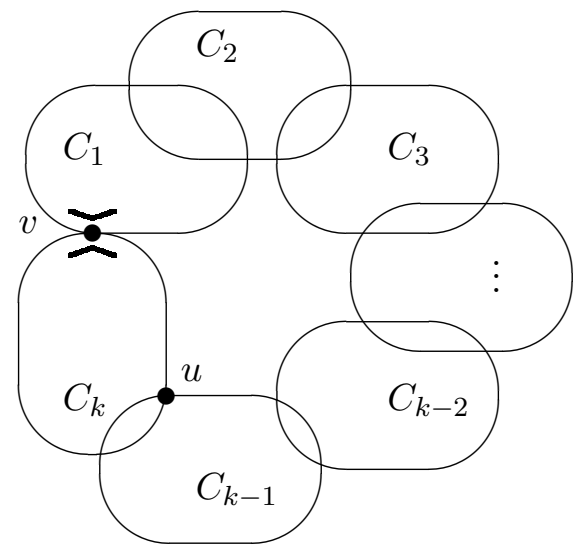

$(G, \mathcal{T})$

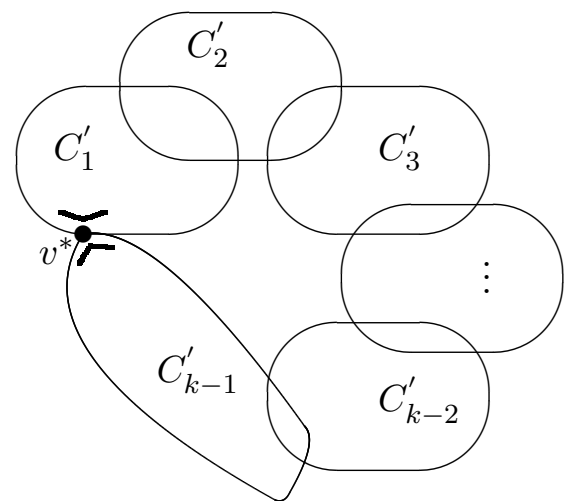

$\left(G^{\prime}, \mathcal{T}^{\prime}\right)$

Figure 1.15: An $\operatorname{ACCCD}(v)$ of $(G, \mathcal{T})$, and, $\left(G^{\prime}, \mathcal{T}^{\prime}\right)$.

Claim 1.5.1. $\mathcal{F}^{\prime}=\left\{H_{1}^{\prime}, H_{2}^{\prime}\right\}$.

Proof of Claim 1.5.1. Assume that $\left|\mathcal{F}^{\prime}\right| \geq 3$. Then we have to show that, for every $C^{\prime} \in$ $\mathcal{F}^{\prime} \backslash\left\{H_{1}^{\prime}, H_{2}^{\prime}\right\}$, the corresponding circuit $C$ in $G$ is a removable circuit of $(G, \mathcal{T})$. It is evident that $C$ is compatible in $(G, \mathcal{T})$ since $v^{*} \notin V\left(C^{\prime}\right)$. We thus want to show that $\left(G \backslash E(C),\left.\mathcal{T}\right|_{G \backslash E(C)}\right)$ has no bad cut-vertex.

To this end, it is sufficient to show that $J$ is 2-connected where $J$ is the subgraph of $G$ induced by the edges of $H_{1}^{\prime}$ and $H_{2}^{\prime}$ and the circuit $C_{k}$. Note that $H_{1}^{\prime} \cup H_{2}^{\prime}$ corresponds in $G$ the $H_{1} \cup H_{2}$ which is a pair of paths with the common end-vertices $u$ and $v$. Adding the circuit $C_{k}$, the resulting graph $J$ is therefore 2-connected (because $H_{1} \cup H_{2} \cup\{u v\}$ is already 2-connected).

It now follows that every CCD of $\left(G^{\prime}, \mathcal{T}^{\prime}\right)$ is a pair of hamiltonian circuits. By the minimality of $(G, \mathcal{T})$, the smaller transitioned graph $\left(G^{\prime}, \mathcal{T}^{\prime}\right)$ is not a counterexample to Theorem 1.1.2. Thus, we can draw the following conclusion.

\section{Claim 1.5.2.}

$$
\left(G^{\prime}, \mathcal{T}^{\prime}\right) \in\langle 2 L\rangle
$$

By Lemma 1.4.3, $(G, \mathcal{T})$ has no digon of type $\lambda>0$. However, by Claim 1.5.2 and Lemma 1.2.1, $\left(G^{\prime}, \mathcal{T}^{\prime}\right)$ contains at least two digons of type $\lambda>0$. Let $D^{\prime}$ be a digon of type $\lambda>0$ in $\left(G^{\prime}, \mathcal{T}^{\prime}\right)$. Because of Lemma 1.4.3, there can only be two kinds of digons in $\left(G^{\prime}, \mathcal{T}^{\prime}\right)$; 
either

$$
E\left(D^{\prime}\right) \cap E\left(C_{k-1}^{\prime}\right) \neq \emptyset \neq E\left(D^{\prime}\right) \cap E\left(C_{k-2}^{\prime}\right)
$$

(which is a virtual digon), or $D^{\prime}$ contains the vertex $v^{*}$ and some edges of $C_{1}^{\prime}$ and $C_{k-1}^{\prime}$, where $k=3$ (which is a real digon).

Let $D_{1}^{\prime}$ be a virtual digon in $\left(G^{\prime}, \mathcal{T}^{\prime}\right)$. Let $D_{1}$ denote the circuit in $G$ corresponding to $D_{1}^{\prime}$. Observe that $C_{k-2}^{\prime} \cap D_{1}^{\prime}=C_{k-2} \cap D_{1}$ is an edge of $G$ and $C_{k-1} \cap D_{1}$ contains some vertices of $C_{k}$. Let $V\left(D_{1}^{\prime}\right)=\{y, z\}$ and let $z$ be an inner vertex of $D_{1}^{\prime}$. If $D_{1}^{\prime}$ is of type 2 , then it can be easily seen that the circuit $C_{k-1} \Delta D_{1}$ is a removable circuit in $(G, \mathcal{T})$. Thus, $D_{1}^{\prime}$ is of type 1 .

Claim 1.5.3. $D_{1}$ is an $\operatorname{ARC}(z)$.

Proof of Claim 1.5.3. Since $D_{1}^{\prime}$ is of type 1 , it is sufficient to show that $G \backslash E\left(D_{1}\right)$ remains 2-connected.

Suppose $G^{*}=G \backslash E\left(D_{1}\right)$ has a cut-vertex, $x$ say. Then $x \in V\left(C_{k-1}\right) \cap V\left(C_{k-2}\right)$, since, for every $i \in\{1, \ldots, k\} \backslash\{k-2, k-1\}, C_{i}$ is also as a circuit in $G^{*}$. For, if $x \notin V\left(C_{k-1}\right) \cap V\left(C_{k-2}\right)$ would hold, then $\{v, x\}$ would be a 2 -vertex-cut in $G$, contradicting Lemma 1.4.5. Note that $J=\left(C_{k-2} \cup C_{k-1}\right) \backslash E\left(D_{1}\right)$ is a pair of edge-disjoint paths with common end-vertices $y$ and $z$ implying that $y$ and $z$ are not cut-vertices of $G^{*}$. Thus, $x \neq y, z$ and $x$ is a cut-vertex of $J$ separating $y$ and $z$. Let $G_{1}^{*}, G_{2}^{*}$ be components of $G^{*} \backslash\{x\}$ with $y \in V\left(G_{1}^{*}\right), z \in V\left(G_{2}^{*}\right)$. Let $K$ be the subgraph of $G^{*}$ induced by the set of circuits $\left\{C_{1}, \ldots, C_{k}\right\} \backslash\left\{C_{k-2}, C_{k-1}\right\}$, which is a connected subgraph of $G^{*}$ since $v \in V\left(C_{1}\right) \cap V\left(C_{k}\right)$. Then it is easy to see that either $V(K) \subseteq V\left(G_{1}^{*}\right) \cup\{x\}$ or $V(K) \subseteq V\left(G_{2}^{*}\right) \cup\{x\}$, but not both. Assume that $V(K) \subseteq V\left(G_{1}^{*}\right) \cup\{x\}$. Then $\{x, z\}$ is a 2-vertex-cut of $G$. This contradicts Lemma 1.4.5 and finishes the proof of the claim.

By the choice of $C_{k}$, the length of $D_{1}$ is not smaller than the length of $C_{k}$. Thus, by Claim 1.5.3, we have the following immediate corollary.

Claim 1.5.4.

$$
V\left(C_{k}\right) \backslash\{v, u\} \subseteq V\left(C_{k-1}\right) \cap V\left(D_{1}\right)
$$

Claim 1.5.5. $k=3$.

Proof of Claim 1.5.5. By Lemma 1.2.1, $\left(G^{\prime}, \mathcal{T}^{\prime}\right)$ has at least two edge-disjoint digons of types 1 or 2. If $k \geq 4$, then every digon of $\left(G^{\prime}, \mathcal{T}^{\prime}\right)$ is virtual. But, by Claim 1.5.4, at least one of them is a digon of type $>0$ in $(G, \mathcal{T})$, contrary to Lemma 1.4.3. Hence $k=3$.

Since $k=3,\left(G^{\prime}, \mathcal{T}^{\prime}\right)$ has at most one virtual digon. Let $D_{2}^{\prime}$ be a real digon in $\left(G^{\prime}, \mathcal{T}^{\prime}\right)$ and let $D_{2}=u v w u$ correspond to $D_{2}^{\prime}$ in $G$.

Claim 1.5.6. $D_{2}$ is an $\mathrm{ARC}(w)$ for some $w \in V\left(C_{1}\right) \cap V\left(C_{2}\right)$. 
Proof of Claim 1.5.6. Denote $D_{2}^{\prime}=\left\langle w, v^{*}\right\rangle$ with one edge in $C_{1}^{\prime}$ and the other edge in $C_{k-1}^{\prime}=C_{2}^{\prime}$. By the definition of $\mathcal{T}^{\prime}\left(v^{*}\right), D_{2}^{\prime}$ is compatible at $v^{*}$. So $w$ is an inner vertex of $D_{2}$ since $D_{2}^{\prime}$ is of type $\lambda>0 . D_{2}^{\prime}$ is extended to $D_{2}$ in $G$ which is the triangle vwuv. If $u$ is also an inner vertex of $D_{2}$, then it is easy to see that $C_{2} \Delta D_{2}$ is a removable circuit in $(G, \mathcal{T})$. Now by Lemma 1.5.2, $D_{2}$ is an $\operatorname{ARC}(w)$.

In the general case, by the analogous argument as we did for $C_{3}$ and $u v$, for every $\operatorname{ARC}\left(v_{1}\right)$, say $C_{v_{1}}=v_{1} v_{2} v_{3} v_{1}$ and the edge $v_{1} v_{2}$, for some $v_{1} \in V(G)$, there exists a vertex $w \in\left(N_{G}\left(v_{1}\right) \cap\right.$ $\left.N_{G}\left(v_{2}\right)\right) \backslash\left\{v_{3}\right\}$ such that $C_{w}=w v_{1} v_{2} w$ is an $\operatorname{ARC}(w)$. This completes the proof of the lemma.

\section{Proof of Theorem 1.1.1'.}

We first claim that every shortest $\mathrm{ARC}$ is a 2-legged cornered triangle. Note that, by Definition 1.5.2, each ARC contains precisely one inner vertex. By Lemma 1.5.3(ii), every shortest ARC is a triangle. That is, every shortest ARC is a 2-legged cornered triangle.

In order to apply Lemma 1.4.10, we further claim that $(G, \mathcal{T})$ has the 2-LTEP. By Lemma 1.5.3(i) and (ii) again, $(G, \mathcal{T})$ contains some 2-legged cornered triangles. By Lemma 1.5.3(iii), each shortest ARC has an extension at every leg.

Thus, by Lemma $1.4 .10,(G, \mathcal{T})$ is exactly the UD- $K_{5}$, which is a contradiction.

\subsubsection{Case II. $(G, \mathcal{T})$ is a counterexample to Theorem 1.1.2.}

Lemma 1.5.4. $(G, \mathcal{T})$ has no non-hamiltonian removable circuit.

Proof. Let $C$ be a non-hamiltonian removable circuit of $(G, \mathcal{T})$. Then the SUD- $K_{5}$-minor-free transitioned graph $\left(G \backslash E(C),\left.\mathcal{T}\right|_{G \backslash E(C)}\right)$ has a $\operatorname{CCD} \mathcal{C}$. Thus, $\mathcal{C} \cup\{C\}$ is a $\operatorname{CCD}$ of $(G, \mathcal{T})$ with at least three circuits, which is a contradiction.

Lemma 1.5.5. $(G, \mathcal{T})$ has no digon of any type.

Proof. Suppose that $D$ is a digon of type $\geq 1$ in $(G, \mathcal{T})$. Let $\left(G^{\prime}, \mathcal{T}^{\prime}\right)=\left(G / D,\left.\mathcal{T}\right|_{G / D}\right)$. It is obvious that every CCD of $(G, \mathcal{T})$ induces a CCD on the smaller graph $\left(G^{\prime}, \mathcal{T}^{\prime}\right)$ because edges of $D$ of are contained in different members of any CCD. By the same token, every CCD of $\left(G^{\prime}, \mathcal{T}^{\prime}\right)$ also induces a $\mathrm{CCD}$ of $(G, \mathcal{T})$. Note that $\left(G^{\prime}, \mathcal{T}^{\prime}\right)$ remains SUD- $K_{5}$-minor-free. Therefore, by the minimality of $(G, \mathcal{T})$, the reduced graph $\left(G^{\prime}, \mathcal{T}^{\prime}\right) \in\langle 2 L\rangle$. Then, by the definition of the family $\langle 2 L\rangle$ of graphs and by the choice of $D$, we have $(G, \mathcal{T}) \in\langle 2 L\rangle$, which is a contradiction.

Assume that $D=\left\langle v_{1}, v_{2}\right\rangle$ is a digon of type 0 in $(G, \mathcal{T})$ with $E(D)=\left\{e_{1}, e_{2}\right\} . \quad D$ is a compatible circuit, but not a removable circuit (by Lemma 1.5.4). Hence, $\left(G \backslash E(D),\left.\mathcal{T}\right|_{G \backslash E(D)}\right)$ has a bad cut-vertex $w$. That is, $\{w\}$ is a 1-separator of $G \backslash E(D)$ separating $G \backslash E(D)$ into two subgraphs $G_{1}$ and $G_{2}$. 
Let $H_{i}=G / G_{j}$ for $i \neq j$ and let $w_{i}$ be the contracted vertex of $G_{i}$, for $i=1,2$. As an eulerian minor of $G$, each $H_{i}$ is SUD- $K_{5}$-minor free. And every CCD $\mathcal{F}_{i}$ of $\left(H_{i},\left.\mathcal{T}\right|_{H_{i}}\right)$ has exactly two members for otherwise, a third member of $\mathcal{F}_{i}$ not passing through the contracted vertex $w_{i}$ is a removable circuit of $(G, \mathcal{T})$, for $i=1,2$. This contradicts Lemma 1.5.4. Hence, $\left(G_{i},\left.\mathcal{T}\right|_{H_{i}}\right)$ remains a Hamilton transitioned graph, and therefore, a member of $\langle 2 L\rangle$. By Lemma 1.2.1, each $\left(G_{i},\left.\mathcal{T}\right|_{H_{i}}\right)$ has at least two edge-disjoint digons of type $\geq 1$, one of which is different from $D$ and must be a digon of the original graph $G$. This contradicts the first part of the proof that $(G, \mathcal{T})$ contains no digon of type $\geq 1$.

Definition 1.5.3. Let $\left\{H_{1}, H_{2}\right\}$ be a CCD of the Hamilton transitioned graph $(G, \mathcal{T})$. A circuit $C=v_{1} v_{2} \ldots v_{k} v_{1}$ is called an $H_{i}$-Segment-Chord Circuit with respect to $v_{1}\left(H_{i}\right.$-SgCC $\left(v_{1}\right)$ for short) if $v_{1} v_{k}$ is a chord of $H_{i}$ and $C \backslash\left\{v_{1} v_{k}\right\}$ is a segment of $H_{i}$ and $v_{1}$ is an inner vertex of $C$ (See Figure 1.16).

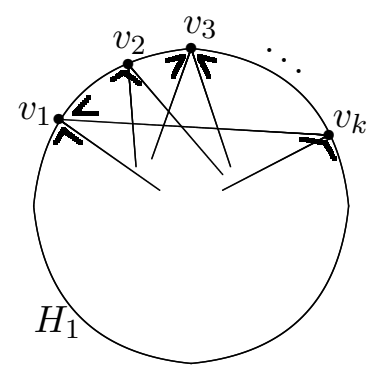

Figure 1.16: $H_{1}-\operatorname{SgCC}\left(v_{1}\right) \quad C_{0}=v_{1} v_{2} \ldots v_{k} v_{1}$.

Obviously, for every compatible hamiltonian circuit $H_{i}$, every transition $P$ at a non-trivial vertex $v$ and every chord $e$ contained in $P$, there exists an $H_{i}-\operatorname{SgCC}(v)$ containing $e$.

Lemma 1.5.6. For any given decomposition $\left\{H_{1}, H_{2}\right\}$ into hamiltonian compatible circuits in $(G, \mathcal{T})$ a shortest $H_{i}$-SgCC is of length 3.

Proof. For $i \in\{1,2\}$, among all $H_{i}$-SgCC's, let $C_{0}=v_{1} \ldots v_{k} v_{1}$ be a shortest one. Without loss of generality $C_{0}$ is an $H_{1}-\mathrm{SgCC}\left(v_{1}\right)$ (see Figure 1.16). By Lemma 1.5.5, $k \geq 3$.

The new 4-regular graph $\left(G^{\prime}, \mathcal{T}^{\prime}\right)$ is obtained from $(G, \mathcal{T})$ by deleting all edges of $C_{0}$ except $v_{1} v_{k}$, contracting $v_{1} v_{k}$ to a new vertex $v^{*}$ and suppressing vertices of degree two. $\left(G^{\prime}, \mathcal{T}^{\prime}\right)$ remains SUD- $K_{5}$-minor-free. Hence, $\left(G^{\prime}, \mathcal{T}^{\prime}\right)$ does have a CCD.

Claim 1.5.7. Every $\operatorname{CCD}$ of $\left(G^{\prime}, \mathcal{T}^{\prime}\right)$ is a pair of hamiltonian circuits.

Let $\mathcal{F}^{\prime}$ be an arbitrary CCD of $\left(G^{\prime}, \mathcal{T}^{\prime}\right)$. There exist two circuits $C_{1}^{\prime}$ and $C_{2}^{\prime}$ in $\mathcal{F}^{\prime}$ each of which contains the new vertex $v^{*}$.

For every circuit $C^{\prime} \in \mathcal{F}^{\prime}$, let $C$ denote the subgraph of $G$ induced by the edges of $C^{\prime}$. Note that $C_{3}=C_{3}^{\prime}$ is also a compatible circuit of $(G, \mathcal{T})$, for every circuit $C_{3}^{\prime} \in \mathcal{F}^{\prime} \backslash\left\{C_{1}^{\prime}, C_{2}^{\prime}\right\}$ if such 
$C_{3}^{\prime}$ exists. We show that $C_{3}$ is removable in $(G, \mathcal{T})$ by showing that the subgraph of $G$ induced by $E\left(C_{0}\right) \cup E\left(C_{1}\right) \cup E\left(C_{2}\right)$ is 2 -connected.

Set $H=G\left[C_{1} \cup C_{2} \cup\left(C_{0} \backslash\left\{v_{1} v_{k}\right\}\right)\right]$; this is the union of three edge-disjoint paths with the common end-vertices $v_{1}$ and $v_{k}$. If $H$ has a cut-vertex $x$, it must separate $v_{1}$ and $v_{k}$. Hence, $H \cup\left\{v_{1} v_{k}\right\}=C_{0} \cup C_{1} \cup C_{2}$ does not have any cut-vertex. Thus, $C_{3}$ is a removable circuit of $(G, \mathcal{T})$, for every circuit $C_{3}^{\prime} \in \mathcal{F}^{\prime} \backslash\left\{C_{1}^{\prime}, C_{2}^{\prime}\right\}$. This contradicts Lemma 1.5.4. Therefore, $\mathcal{F}^{\prime}=\left\{C_{1}^{\prime}, C_{2}^{\prime}\right\}$. Since $\left(G^{\prime}, \mathcal{T}^{\prime}\right)$ has no SUD- $K_{5}$-minor, by the minimality of $(G, \mathcal{T})$, we draw the following conclusion.

Claim 1.5.8. $\left(G^{\prime}, \mathcal{T}^{\prime}\right) \in\langle 2 L\rangle$.

Note that $v^{*}$ is the only contracted vertex of $G^{\prime}$ and $v_{2}, \ldots, v_{k-1}$ are the only suppressed vertices of $G^{\prime}$. Since $G$ has no digon of type $\lambda>0$ (see Lemma 1.5.5), for each digon $D^{\prime}$ of $G^{\prime}$, the corresponding circuit $D$ of $G$ must contain either some of $\left\{v_{2}, \ldots, v_{k-1}\right\}$ or the edge $v_{1} v_{k}$. And if $D$ contains $v_{1} v_{k}$, then $D^{\prime}$ must contain the contracted vertex $v^{*}$ and be compatible at $v^{*}$.

Claim 1.5.9. Let $D^{\prime}$ be a digon of type $\lambda>0$ in $G^{\prime}$. Then the corresponding circuit in $G$ is an $\mathrm{H}_{2}$-SgCC.

If $x$ is an inner vertex of $D^{\prime}=\langle x, y\rangle$, then one edge of $D^{\prime}$ is an $H_{1}$-edge, another one is an $H_{2}$-segment. So it is an $H_{2}-\operatorname{SgCC}(x)$.

Assume that $k \geq 4$.

Claim 1.5.10. There is no real digon in $G^{\prime}$.

Suppose to the contrary that there is a real digon $D^{\prime}$ in $G^{\prime}$. Let $D$ be the circuit in $G$ corresponding to $D^{\prime}$. Since $D$ is not a digon in $G$ and does not contain any vertex of $\left\{v_{2}, \ldots, v_{k-1}\right\}$, it corresponds to a $H_{2}-\operatorname{SgCC}(x)$ of length 3 . This contradicts $k \geq 4$.

Claim 1.5.11. Every virtual digon uses $v^{*}$.

Let $D_{1}^{\prime}, D_{2}^{\prime}$ be a pair of edge-disjoint digons of $G^{\prime}$; both are virtual (by Claim 1.5.10).

Suppose that $v^{*} \notin V\left(D_{1}^{\prime}\right)$ and $x$ is an inner vertex of $D_{1}^{\prime}$. By Claim $1.5 .9, D_{1}$ is an $H_{2^{-}}$ $\operatorname{SgCC}(x)$. By the choice of $C_{0}$ (that it is shortest), $D_{1}$ must contain all vertices of $\left\{v_{2}, \ldots, v_{k-1}\right\}$. Thus $D_{2}$ contains no other suppressed vertices and, therefore, $D_{2}^{\prime}$ is a real digon contradicting Claim 1.5.10.

Claim 1.5.12. Every virtual digon is compatible at $v^{*}$.

Suppose that $v^{*}$ is an inner vertex of the digon $D_{1}^{\prime}$. Thus, $D_{1}$ is an $H_{2}-\operatorname{SgCC}\left(v_{1}\right)$. We will show that $D_{1}$ is shorter than $C_{0}$. Since $D_{1}^{\prime}$ and $D_{2}^{\prime}$ are edge-disjoint, each of $D_{1}^{\prime}, D_{2}^{\prime}$ contains one transition of $\mathcal{T}^{\prime}\left(v^{*}\right)$. Hence, $v^{*}$ must be an inner vertex of both $D_{1}^{\prime}$ and $D_{2}^{\prime}$. Furthermore, the corresponding circuits $D_{1}, D_{2}$ in $G$ do not contain the chord $v_{1} v_{k}$, and contain some vertex 
of $\left\{v_{2}, \ldots, v_{k-1}\right\}$. That is, $D_{1}$ contains at most $(k-3)$ vertices of $\left\{v_{2}, \ldots, v_{k-1}\right\}$. Thus, $D_{1}$ is shorter than $C_{0}$. This contradicts the choice of $C_{0}$.

Claim 1.5.13. $k \leq 4$. Furthermore, each $D_{i}$ contains precisely one vertex of $\left\{v_{2}, v_{3}\right\}$ if $k=4$.

Let $D_{1}^{\prime}, D_{2}^{\prime}$ be two edge-disjoint digons of $G^{\prime}$. Both are virtual, use $v^{*}$ and are compatible at $v^{*}$. And it is obvious that if $D_{1}^{\prime}$ traverses $v_{n}$ and then $D_{2}^{\prime}$ traverses $v_{k+1}$. The corresponding circuits $D_{i}$ in $G$ contain an $H_{2}$-segment each passing through at least $k-3$ vertices of $\left\{v_{2}, \ldots, v_{k-1}\right\}$, $i=1,2$; for otherwise, it would be shorter than $C_{0}$. Since $G$ is 4 -regular, $(k-3)+(k-3) \leq k-2$. Thus, $k \leq 4$ and $\left\{v_{2}, \ldots, v_{k-1}\right\}=\left\{v_{2}, v_{3}\right\}$ implying the validity of the remainder of the claim.

Claim 1.5.14. $k=3$.
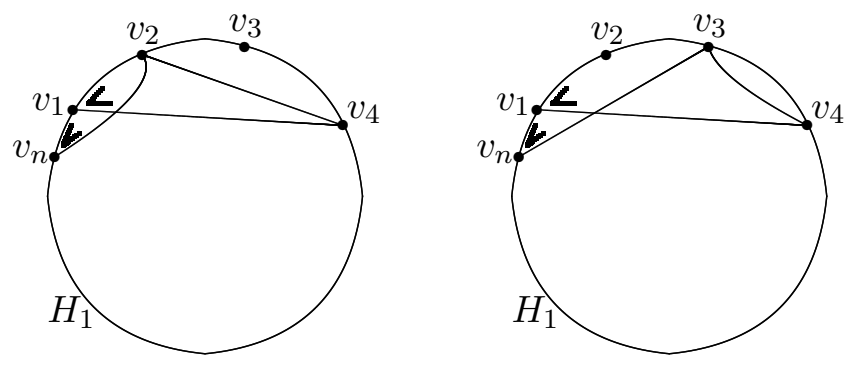

Figure 1.17: $k=4: \quad D_{1}=v_{1} v_{4} v_{\mu} v_{n} v_{1}, \quad \mu=2,3$.

If $k=4$, then, by Claim 1.5.13, let $D_{1}=v_{1} v_{4} v_{\mu} v_{n} v_{1}$ with an inner vertex $v_{n}$ where $\mu=2$ or 3 (see Figure 1.17). Furthermore, the segment $v_{4} v_{\mu} v_{n}$ is an $H_{2}$-segment. If $\mu=2$, then there is a triangle $v_{n} v_{2} v_{1} v_{n}$ inner at $v_{n}$, which is an $H_{1}-\operatorname{SgCC}\left(v_{n}\right)$ shorter than $C_{0}$. If $\mu=3$, then $D^{*}=\left\langle v_{3}, v_{4}\right\rangle$ induces a digon of $G$. This contradicts Lemma 1.5.5. Thus, $k=3$ and Lemma 1.5.6 now follows.

Since $k=3$ and by Claim 1.5.8, at least one digon of $\left(G^{\prime}, \mathcal{T}^{\prime}\right)$ is a real digon, with the circuit corresponding to this digon in $(G, \mathcal{T})$ is a 1-legged triangle $v_{1} v_{3} w v_{1}$ with the corner $w$ and a leg either $v_{1} w$ or $v_{3} w$.

In Lemma 1.5.6, we proved the existence of 1-legged triangles. In the next lemma (Lemma 1.5.7), we show that every 1-legged triangle has the 1-LTEP. Note that the proof of this lemma is similar to the proof of Claims 1.5.7 and 1.5.8 for Lemma 1.5.6.

Lemma 1.5.7. $(G, \mathcal{T})$ has the 1-LTEP.

Proof. Assume that $S_{1}=u_{1} u_{2} u_{3} u_{1}$ is a 1-legged triangle with the corner $u_{1}$ and a leg $u_{1} u_{3}$. Let $\left(G^{\prime \prime}, \mathcal{T}^{\prime \prime}\right)$ be a new 4 -regular graph obtaining from $(G, \mathcal{T})$ as follows. Remove $u_{1} u_{2}$ and $u_{2} u_{3}$, contract $u_{1} u_{3}$ to a new vertex $u^{*}$ and then suppress vertices of degree two. $\left(G^{\prime \prime}, \mathcal{T}^{\prime \prime}\right)$ remains SUD- $K_{5}$-minor-free. 
Claim 1.5.15. $\left(G^{\prime \prime}, \mathcal{T}^{\prime \prime}\right)$ has no bad cut-vertex.

Proof of Claim 1.5.15. Suppose that $p$ is a bad cut-vertex in $\left(G^{\prime \prime}, \mathcal{T}^{\prime \prime}\right)\left(p \neq u_{3}\right.$, otherwise $u_{1}$ is a cut-vertex of $G$ contrary to $G$ is 2 -connected). Thus, $\left\{u_{2}, p\right\}$ is a 2 -vertex-cut in $(G, \mathcal{T})$. Let $G_{1}^{\prime \prime}$ and $G_{2}^{\prime \prime}$ be the components of $G \backslash\left\{u_{2}, p\right\}$ such that $\left\{u_{1}, u_{3}\right\} \subseteq V\left(G_{1}^{\prime \prime}\right)$.

Remove $V\left(G_{2}^{\prime \prime}\right)$ and identify $u_{2}$ and $p$ to a new vertex $q$ to obtain a new transitioned 4-regular graph $\left(G^{\prime \prime \prime}, \mathcal{T}^{\prime \prime \prime}\right)$ which is admissible (since $u_{1} u_{3} \in E(G)$, ) and SUD- $K_{5}$-minor-free. Thus $\left(G^{\prime \prime \prime}, \mathcal{T}^{\prime \prime \prime}\right)$ has a CCD. It is easily seen that every CCD of $\left(G^{\prime \prime \prime}, \mathcal{T}^{\prime \prime \prime}\right)$ is a pair of hamiltonian circuits (a removable circuit in $\left(G^{\prime \prime \prime}, \mathcal{T}^{\prime \prime \prime}\right)$ not containing $q$ is also a removable circuit in $(G, \mathcal{T})$ ). By the choice of $(G, \mathcal{T}),\left(G^{\prime \prime \prime}, \mathcal{T}^{\prime \prime \prime}\right) \in\langle 2 L\rangle$. By Lemma 1.2.1, $\left(G^{\prime \prime \prime}, \mathcal{T}^{\prime \prime \prime}\right)$ has two edge-disjoint digons of type $>0$. Since $(G, \mathcal{T})$ has no digon of any type, $\left\{u_{1} u_{2}, u_{1} p\right\} \in \mathcal{T}\left(u_{1}\right)$. However, $\left\{u_{1} u_{2}, u_{1} u_{3}\right\} \in \mathcal{T}\left(u_{1}\right)$ (see definition of a 1-legged triangle with corner $u_{1}$ ); this contradicts $p \neq u_{3}$. Now Claim 1.5.15 follows.

Hence, $\left(G^{\prime \prime}, \mathcal{T}^{\prime \prime}\right)$ does have a CCD.

Claim 1.5.16. $\left(G^{\prime \prime}, \mathcal{T}^{\prime \prime}\right) \in\langle 2 L\rangle$.

Let $\mathcal{F}^{\prime \prime}$ be an arbitrary $\operatorname{CCD}$ of $\left(G^{\prime \prime}, \mathcal{T}^{\prime \prime}\right)$. There exist two circuits $C_{1}^{\prime \prime}$ and $C_{2}^{\prime \prime}$ in $\mathcal{F}^{\prime \prime}$ each of which contains the new vertex $u^{*}$.

For every circuit $C^{\prime \prime} \in \mathcal{F}^{\prime \prime}$, denote bz $C$ the subgraph of $G$ induced by the edges of a circuit $C^{\prime \prime}$. Note that $C_{3}$ is also a compatible circuit of $(G, \mathcal{T})$, for every circuit $C_{3}^{\prime \prime} \in \mathcal{F}^{\prime \prime} \backslash\left\{C_{1}^{\prime \prime}, C_{2}^{\prime \prime}\right\}$.

Let $H$ be the subgraph of $G$ induced by the edges contained in $C_{1}, C_{2}$ and $\left\{u_{1} u_{3}\right\}$, which is the union of three edge-disjoint paths with the common end-vertices $u_{1}$ and $u_{3}$; and it is 2 -connected. Hence, $S_{1} \cup C_{1} \cup C_{2}$ is 2 -connected. Thus, $C_{3}$ is a removable circuit of $(G, \mathcal{T})$, for every circuit $C_{3}^{\prime \prime} \in \mathcal{F}^{\prime \prime} \backslash\left\{C_{1}^{\prime \prime}, C_{2}^{\prime \prime}\right\}$ which contradicts Lemma 1.5.4. Therefore, $\mathcal{F}^{\prime \prime}=\left\{C_{1}^{\prime \prime}, C_{2}^{\prime \prime}\right\}$.

Note that $\left(G^{\prime \prime}, \mathcal{T}^{\prime \prime}\right)$ has no SUD- $K_{5}$-minor, thus by the minimality of $(G, \mathcal{T})$, we have $\left(G^{\prime \prime}, \mathcal{T}^{\prime \prime}\right) \in\langle 2 L\rangle$ which finishes the proof of the claim.

By Lemma 1.2.1, $\left(G^{\prime \prime}, \mathcal{T}^{\prime \prime}\right)$ has at least two edge-disjoint digons of type $\lambda>0$. Since $(G, \mathcal{T})$ has no digon by Lemma 1.5.5, for each digon $D^{\prime \prime}$ of $\left(G^{\prime \prime}, \mathcal{T}^{\prime \prime}\right)$, the corresponding circuit $D$ in $G$ must contain either $u_{2}$ or the edge $u_{1} u_{3}$.

There is at most one $D$ in $(G, \mathcal{T})$ with $u_{2} \in V(D)$ corresponding to a digon in $\left(G^{\prime \prime}, \mathcal{T}^{\prime \prime}\right)$; otherwise, $(G, \mathcal{T})$ would contain a digon, contrary to Lemma 1.5.5. Let $D^{\prime \prime}=\left\langle u^{*}, w\right\rangle$ be a digon of type $>0$ in $\left(G^{\prime \prime}, \mathcal{T}^{\prime \prime}\right)$ containing the contracted vertex $u^{*}$ with edges $\left\{e_{1}, e_{2}\right\}$ (such digon must exist because of the preceding argument). Because of Lemma 1.5.5 $u^{*}$ is not an inner vertex of $D^{\prime \prime}$. Its corresponding triangle $D$ in $G$ containing the edge $u_{1} u_{3}$ and therefore $\left\{e_{1}, e_{2}\right\}$ is not a transition in $\mathcal{T}\left(u^{*}\right)$. Therefore, the only inner vertex of $D^{\prime \prime}$ is $w$. Thus $(G, \mathcal{T})$ has the 1-LTEP. 


\section{Proof of Theorem 1.1.2.}

By Lemma 1.5.7, $(G, \mathcal{T})$ has the 1-LTEP. Thus by Lemma 1.4.11, either $(G, \mathcal{T})$ is the UD- $K_{5}$ or it has a CCD of size 3, which is a contradiction. Now Theorem 1.1.2 follows. 


\section{Chapter 2}

\section{Embedding signed flows}

From now on, we study the integer flows of signed graphs. Tutte establishd an equivalent relation between integer flows of planar graphs and face coloring problems. As a generalization, we introduce the natural signatures of all embedded signed graphs and study the existence of integer flows, from which we generalize the equivalent relation from planar cases to all possible embeddings, including the non-orientable cases.

In 1983, Bouchet proposed a conjecture that every flow-admissible signed graph admits a nowhere-zero 6-flow. In this paper, We deduce this conjecture to a small class of embedded graphs by applying the classification theorem of surfaces. Moreover, we verify this conjecture for a special but important case in this class of embedded graphs, which can be view as a new approach towards Bouchet't conjecture.

\section{$2.1 \quad$ Introduction}

Motivated by face coloring problems, such as the famous Four color problem, Tutte introduced integer flows. The following equivalent relations between these two categories of problems indicates that integer flows is no doubt a powerful tool to deal with the face coloring problems.

Theorem 2.1.1. (Tutte [28]) Let $G$ be a graph strongly embedded on an orientable surface $S$. If $G$ is $k$-face colorable on $S$ then $G$ admits a nowhere-zero $k$-flow. Furthermore, if $S$ is a sphere, then they are equivalent.

In this paper, we generalized this relation to all the surfaces (including non-orientable cases) and introduce the natural signature of embedded graphs.

Theorem 2.1.2. Let $G$ be a signed graph strongly embedded on a surface $S$ and $\sigma$ be the natural signature with respect to the embedding. If $G$ is $k$-face colorable on $S$ then $(G, \sigma)$ admits a nowhere-zero $k$-flow. Furthermore, if $S$ is a sphere or a projective plane, then they are equivalent. 
Basic definitions will be introduced in Section 2.2. For more terminology and notations not defined here we refer to [7]. Actually, the signature of a signed graph can be defined arbitrarily, which gives a natural generalization of the ordinary graphs. Indeed, Bouchet proposed the following famous conjecture on the flows of signed graphs and it remains open.

Conjecture 2.1.1. (Bouchet [5]) Every flow-admissible signed graph admits a nowhere-zero 6-flow.

The main approach of this paper is to deduce the Conjecture 2.1.1 to a special case of embedded graphs. We will make use of the methods of surfaces, more precisely, the isomorphic operations of surfaces. Since the negative edges of a natural signature are caused by cross-caps of the surface, which we will see later in next section, we want to reduce them as much as possible. Indeed, we have the following proposition by reversing the proof of the Classification Theorem for surfaces.

Proposition 2.1.1. Every surface is homeomorphic to a space obtained from the sphere by adding $n$ tori and $m$ cross-caps with $m \leq 2$ and $n \geq 0$.

The core method of isomorphic operation, called cut-paste operation, is frequently used in the proof of Proposition 2.1.1, as well as in the Classification Theorem. We next show that the existence of nowhere-zero flow is kept under these operations.

Theorem 2.1.3. Let $S$ be a surface and $(G, S, \pi)$ be an embedded graph. Then the two natural signatures are equivalent if one of corresponding surfaces can be obtained from the other by cut-paste operation.

Combining these result together, we get the following

Theorem 2.1.4. Bouchet's Conjecture holds if every graph embedded on a surface with at most 2 cross-cap admits a nowhere-zero 6-flow for its natural signature.

The structure of the paper is organized as follows: Theorem 2.1.2, Proposition 2.1.1 and Theorem 2.1.3 will be proved in Section 2.2. In Section 2.3, we construct the nowhere-zero 6-flow for an important case of Theorem 2.1.4.

\subsection{Main result: the methods of surfaces operations}

\subsubsection{Notation and terminology}

Let $G=(V, E)$ be a graph. For $U \subseteq V(G)$, denote $\delta_{G}(U)$ the set of edges with one end in $U$ and the other in $V \backslash U$. We always skip the subscript $G$ if it is clear from the context and simplify $\delta_{G}(\{v\})$ by $\delta_{G}(v)$. 
A signed graph $(G, \sigma)$ is a pair consisting of a graph $G$ together with a signature $\sigma: E(G) \rightarrow$ $\{ \pm 1\}$. For convenience, the signature $\sigma$ is always omitted if no confusion arises. An edge $e \in E(G)$ is positive if $\sigma(e)=1$ and negative otherwise. Denote the set of all negative edges of $G$ by $E_{N}(G)$. A graph is called unsigned if $E_{N}(G)=\emptyset$. For a vertex $v$ in $G$, we define a new signature $\sigma^{\prime}$ by changing $\sigma^{\prime}(e)=-\sigma(e)$ for each $e \in \delta_{G}(v)$. We say that $\sigma^{\prime}$ is obtained from $\sigma$ by making a switch at the vertex $v$. Two signatures are said to be equivalent if one can be obtained from the other by making a sequence of switching operations.

Every edge of $G$ is composed of two half-edges $h$ and $\hat{h}$, each of which is incident with one end. Denote the set of half-edges of $G$ by $H(G)$ and the set of half-edges incident with $v$ by $H_{G}(v)$. For a half-edge $h \in H(G)$, we refer to $e_{h}$ as the edge containing $h$. An orientation of a signed graph $(G, \sigma)$ is a mapping $\tau: H(G) \rightarrow\{ \pm 1\}$ such that $\tau(h) \tau(\hat{h})=-\sigma\left(e_{h}\right)$ for each $h \in H(G)$. It is convenient to think of $\tau$ as an assignment of orientations on $H(G)$. Namely, if $\tau(h)=1, h$ is a half-edge oriented away from its end and otherwise towards its end. Such an ordered triple $(G, \sigma, \tau)$ is called a bidirected graph.

Definition 2.2.1. Assume that $G$ is a signed graph associated with an orientation $\tau$. Let $A$ be an abelian group and $f: E(G) \rightarrow A$ be a mapping. The boundary of $f$ at a vertex $v$ is defined as

$$
\partial f(v)=\sum_{h \in H_{G}(v)} \tau(h) f\left(e_{h}\right) .
$$

The pair $(\tau, f)$ (or simplify, $f)$ is an A-flow of $G$ if $\partial f(v)=0$ for each $v \in V(G)$, and is an (integer) $k$-flow if it is a $\mathbb{Z}$-flow and $|f(e)|<k$ for each $e \in E(G)$. Let $f$ be a flow of a signed graph $G$. The support of $f$, denoted by $\operatorname{supp}(f)$, is the set of edges e with $f(e) \neq 0$. The flow $f$ is nowhere-zero if $\operatorname{supp}(f)=E(G)$.

An embedding of a graph $G$ on surface $S$ is an injective continuous function $\pi$ from $G$ to $S$ such that vertices corresponds to distinct points of $S$ (called vertex-point) and each edge corresponds a path of $S$ joining its two vertex-points, which satisfies that different paths can only have intersection at vertex-points. We use the triple $(G, \pi, S)$ to denote the embedded graph. Each component of $S-G$ is a face of $G$ and denoted by $F(G)$ the set of all faces of $G$. An embedding is called strong if the boundary of each face is circuit. Clearly each edge is incident with two different faces if the embedding is strong. For a strong embedding, a $k$-face coloring is a map $c: F(G) \rightarrow\{1, \ldots, k\}$ and $c$ is called proper if each edge lies between two differently colored faces. $G$ is called $k$-colorable (for the embedding on $S$ ) if $G$ has a $k^{\prime}$-face coloring for some integer $k^{\prime} \leq k$.

\subsubsection{Face coloring for non-orientable surfaces}

As a special case of signed graphs, the natural signature is defined as follows. 
Definition 2.2.2. (See Mohar-Thomassen's book [22]) Let $G$ be a graph strongly embedded on a surface $S$. A natural signature $\sigma$ with respect to the embedding is a mapping $\sigma: E(G) \rightarrow\{ \pm 1\}$ that $\sigma(e)=-1$ if and only if e passes through the cross-caps of $S$ odd times.

In fact, arbitrarily a signature of a graph can be viewed as a natural signature induced by some surface and a corresponding embedding: Let $(G, \sigma)$ be a signed graph. We may firstly draw the ordinary graph $G$ on the sphere with some possible crossings. Next insert a cross-cap at each crossing and make this drawing an embedding. That is, delete a small open disk centred at the crossing point and paste each pair of diametrical points on the boundary of the deleted open disk, which is a circle. Finally, insert possibly one cross-cap at the segments of each edge, making the signature of each edge compatible with the parity of cross-caps it passes. Thus we show

Proposition 2.2.1. For arbitrarily a signed graph $(G, \sigma)$, there exists a surface $S$ and an embedding of $G$ on $S$ such that $\sigma$ is the nataral signature of the embedding.

The concept of natural signatures enables us to extend Tutte's flow theory from orientable surfaces to non-orientable surfaces.

Proof of Theorem 2.1.2. We may assume that the vertex set of $G$ has no intersection with the boundaries of the cross-caps after a proper adjustment of the embedding. Let $c$ be a proper face coloring of the embedded graph $G$, i.e., a map from all the regions of $S$ to $\{1, \ldots, k\}$, we next want to construct a nowhere-zero $k$-flow of $G$. By the Classification Theorem of surfaces, $S$ can be obtained from the sphere by adding several tori and cross-caps. Cut along each cross-cap, i.e., replace each cross-cap by a deleted open disk on the sphere. We get an orientable structure $S^{\prime}$.

Let $D$ be an arbitrary orientation of $G$. Define a function $f: H(G) \rightarrow \mathbb{Z}$ by setting $f(h)=$ $c\left(F_{1}\right)-c\left(F_{2}\right)$ where $F_{1}\left(F_{2}\right)$ is the face lies on the left (right) side of $h$ (note that $S^{\prime}$ is already orientable).

We claim that $f\left(h_{1}^{e}\right)=f\left(h_{1}^{e}\right)$ for any $e \in E(G)$. If an edge passes through a cross-cap, its adjacent face will change side. By definition, a negative edge $e$ passes through an odd number of cross-caps, hence each of the two faces adjacent with $e$ switches side odd times along $e$. Thus each face lies on the same side of $h_{1}^{e}$ and $h_{2}^{e}$, hence $f\left(h_{1}^{e}\right)=f\left(h_{1}^{e}\right)$. Similarly for the case of positive edges.

So we may view $f$ as a function $f: E(G) \rightarrow \mathbb{Z}$ by setting $f(e)=f\left(h_{1}^{e}\right)=f\left(h_{1}^{e}\right)$ Clearly $f$ satisfies the Kirchhoff's Law at each vertex and $1<|f(e)|<k-1$ since $c$ is a proper face coloring. Thus $f$ is a nowhere-zero $k$-flow of $G$.

If $S$ is a sphere, then the graph is unsigned since there is no cross-cap in $S$, thus go back to the case of Theorem 2.1.1. 
Finally, consider $S$ be a projective plane and $f$ be the nowhere-zero $k$-flow $f$ of $G$. Construct a surface $S^{\prime \prime}$ by contracting the cross-cap of $S$ into a single point and $G^{\prime}$ by insert a new vertex $v_{0}$ to $G$ at that point. Verify that $f$ is still a flow of $G^{\prime}$ by showing that $\partial f\left(v_{0}\right)=-\sum_{v \in V} \partial f(v)=0$. Noting that $S^{\prime \prime}$ is already a sphere. We can use Tutte's method in Theorem 2.1.1 to define a face coloring $c^{\prime}$ of $G^{\prime}$ : Pick arbitrarily a face $F_{0}$ of $S^{\prime}$ and set $c^{\prime}\left(F_{0}\right)=0$. For any edge $e$ together with its two incident faces $F^{\prime}$ and $F^{\prime \prime}$ such that $F^{\prime}$ is on the left, set

$$
c\left(F^{\prime \prime}\right)=c\left(F^{\prime}\right)+f(e) \quad(\bmod k)
$$

To verify that $c^{\prime}$ is well-defined, we only need to show that each pair of diametrical faces at $v_{0}$ (i.e., the two faces between the same adjacent pair of negative edges of $G$ ) are assigned the same color. Let $F_{1}, F_{2}$ be such two faces. Suppose that $F_{1}$ is already colored and then we color the faces incident with $v_{0}$ recursively in the cyclic order. We pass through exactly half of $E\left(v_{0}\right)$ before reaching $F_{2}$, thus each negative edge of $G$ is involved exactly once in this process. Since $\partial f\left(v_{0}\right)=-\sum_{v \in V} \partial f(v)=0$, we have $c^{\prime}\left(F_{1}\right)-c^{\prime}\left(F_{2}\right)= \pm \frac{1}{2} \partial f\left(v_{0}\right)=0$.

\subsection{3 topological methods}

A surface is a connected and compact topological space such that each point has a neighbourhood homeomorphic to the open disk. Most notations we follow Munkres's book [23] with a little simplification.

Perhaps the most natural example of surface is the quotient space obtained by pasting the edges of a polygonal region pair by pair. Actually, all the surfaces can be obtained in this way by the well-known Classification Theorem.

More precisely, we introduce the polygon representation and cut-paste operation, which are core methods both in Classification and our project.

A polygon representation consists of the following data: (1) a polygon region in $\mathbb{R}_{2}$ with even number of edges. (2) each edge is assigned a label such that each label appears exactly twice. (3) each label is given a exponent +1 or -1 to indicate its orientation along clockwise. Every polygon representation can be written as a label scheme, i.e, $w=\left(a_{1}\right)^{ \pm 1} \cdots\left(a_{n}\right)^{ \pm 1}$, which is a list of all the labels together with their orientations in clockwise or counter-clockwise.

We say a surface $S$ has a polygon representation (or equivalently, a label scheme) if $S$ can be obtained from the polygon representation by paste each pair of edges with same label along their orientations.

Definition 2.2.3. Let $P_{1}, P_{2}$ be two polygon representations, we define the cut-paste operation if $P_{2}$ can be obtained from $P_{1}$ by following steps: Let $L$ be a segment of $P_{1}$ joining two nonadjacent vertices and a be a pair of labels lying on different side of $L$. Cut the polygon $P_{1}$ into 2 pieces along $L$ and paste at 2 edges with label a along their orientations. Give the new pair of 

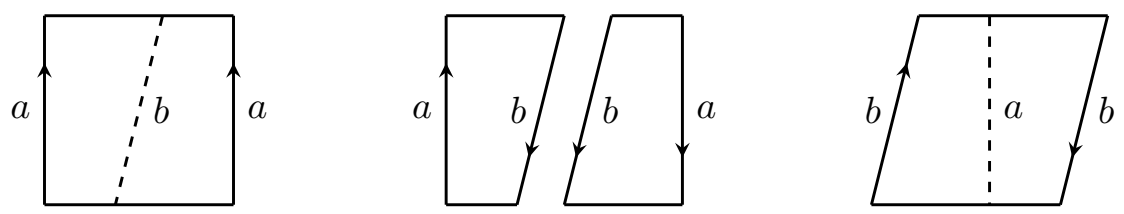

Figure 2.1: Cut-paste operation.

edges $L$ a new label $b$. If the pair a have same (resp., opposite) orientation, the pair $b$ are also assigned same (resp., opposite) orientation. Thus we get a new polygon representation $P_{2}$. Such a cut-paste operation is said of type-I (resp., type-II) if the pair a have same (resp., opposite) orientation. See Figure 2.1.

Note that one of the two pieces should be flipped before pasting for the type-I operation.

Definition 2.2.4. The surface obtained from the following labelling scheme $w_{t}$ is called the $n$-fold torus and denoted by $T_{n}$ where

$$
w_{t}=\left(a_{1} b_{1} a_{1}^{-1} b_{1}^{-1}\right)\left(a_{2} b_{2} a_{2}^{-1} b_{2}^{-1}\right) \ldots\left(a_{n} b_{n} a_{n}^{-1} b_{n}^{-1}\right)
$$

with $n \leq 1$. For convenience reason, let $T_{0}=S^{2}$ be the sphere.

Definition 2.2.5. The surface obtained from the following labelling scheme $w_{p}$ is called the $m$-fold projective plane and denoted by $P_{m}$ where

$$
w_{t}=\left(a_{1} a_{1}\right)\left(a_{2} a_{2}\right) \ldots\left(a_{m} a_{m}\right)
$$

with $m \leq 1$.

Theorem 2.2.1. (Classification theorem) Every surface is homeomorphic either to the $n$-fold torus $T_{n}(n \geq 0)$ or to the $m$-fold projective plane $P_{m}(m \geq 1)$.

One can easily check that all the vertices of the polygon in the standard forms Definition 2.2.4 or Definition 2.2.5 are glued to a single point in the corresponding surfaces. This property is kept under cut-paste operation.

From the definition of natural signature, we know that the cross-caps corresponds the negative edges. We hope that they occur as less as possible. The core method to prove the Classification Theorem is the isomorphic operation, i.e., cut-paste operation. We use the same operation and somehow reverse the process of Classification Theorem, we can get a proof of Proposition 2.1.1.

Proof of Proposition 2.1.1. Let $w$ be a labelling scheme and $\left[w_{1}\right]\left[w_{2}\right],\left[w_{3}\right]\left[w_{4}\right]$ be two subsequence of $w$. For a cut-paste operation, we call it cutting along $\left[w_{1}\right] \bullet\left[w_{2}\right]$ and $\left[w_{3}\right] \bullet\left[w_{4}\right]$ 


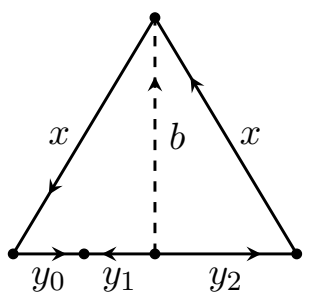

(1)

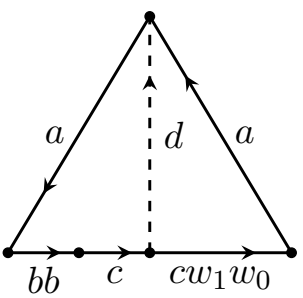

(1)

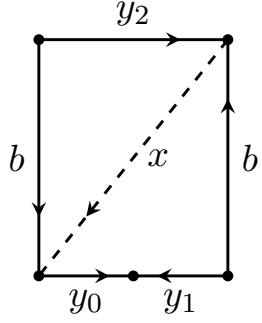

(2)

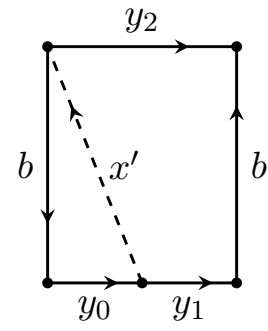

(3)

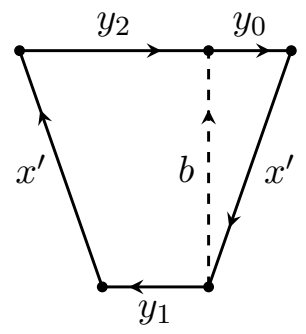

(4)

Figure 2.2:

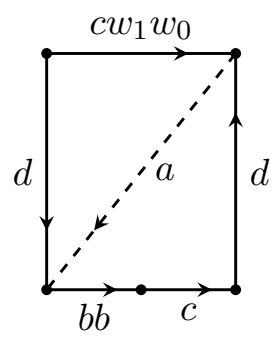

(2)

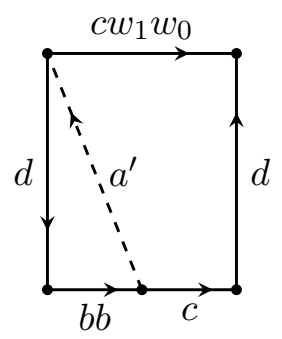

(3)

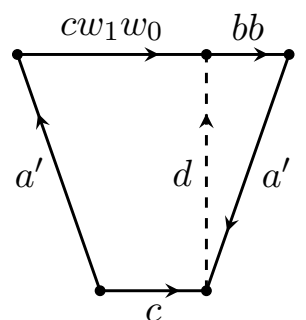

(4)

Figure 2.3:

if the cutting segment goes from the common vertex of $\left[w_{1}\right]$ and $\left[w_{2}\right]$ to the common vertex of $\left[w_{3}\right]$ and $\left[w_{4}\right]$.

Step1. We show that the following two labeling scheme are equivalent. $[x x]\left[y_{0} y_{1}^{-1} y_{2}\right] \sim$ $\left[y_{0}\right] x^{\prime}\left[y_{1}\right] x^{\prime}\left[y_{2}\right]$ (equation 1). As shown in Figure 2.2, starting from $[x x]\left[y_{0} y_{1}^{-1} y_{2}\right]$, we firstly cut along $[x] \bullet[x]$ and $\left[y_{0} y_{1}^{-1}\right] \bullet\left[y_{2}\right]$ with a new label $b$ and paste at the pair $x$. Next cut along $\left[y_{0}\right] \bullet\left[y_{1}\right]$ and $\left[y_{2}^{-1}\right] \bullet[b]$ with a new label $x^{\prime}$ and paste at the pair $b$, we get $\left[y_{0}\right] x^{\prime}\left[y_{1}\right] x^{\prime}\left[y_{2}\right]$.

Step2. Next we show that $\left[w_{0}\right](a a b b c c)\left[w_{1}\right] \sim\left[w_{0}\right]\left(c^{\prime} c^{\prime}\right)\left(a^{\prime} b^{\prime} a^{\prime-1} b^{-1}\right)\left[w_{1}\right]$ (equation 2): Applying equation 1 to $\left[w_{0}\right](a a b b c c)\left[w_{1}\right]$ by setting $x=a$ and $y_{0}=b b, y_{1}=c^{-1}, y_{2}=c w_{1} w_{0}$, we get an equivalent labelling scheme $[b b] a^{\prime}\left[c^{-1}\right] a^{\prime} c w_{1} w_{0}$, see Figure 2.3. Next let $x=b$ and $y_{0}=a^{\prime}$, $y_{1}=c^{-1}, y_{2}=a^{\prime} c w_{1} w_{0}$, we get $\left[a^{\prime}\right] b^{\prime}[c] b^{\prime}\left[a^{\prime} c w_{1} w_{0}\right]$, see Figure 2.4

If at least one of $w_{0}, w_{1}$ is not empty, as shown in Figure 2.5, then by reversing equation 1 and setting $x=c$ and $y_{0}=a^{\prime} b^{\prime}, y_{1}=b^{\prime} a^{\prime}, y_{2}=w_{1} w_{0}$, we get $\left[c^{\prime} c^{\prime}\right]\left[a^{\prime} b^{\prime}\right]\left[\left(b^{\prime} a^{\prime}\right)^{-1}\right]\left[w_{1} w_{0}\right]=$ $\left[w_{0}\right]\left(c^{\prime} c^{\prime}\right)\left(a^{\prime} b^{\prime} a^{-1} b^{-1}\right)\left[w_{1}\right]$. Done. If both $w_{0}$ and $w_{1}$ are empty, as shown in Figure 2.6, then we cut $\left[a^{\prime}\right] b^{\prime}[c] b^{\prime}\left[a^{\prime} c\right]=\left[a^{\prime} b^{\prime}\right] c\left[b^{\prime} a^{\prime}\right] c$ along $c \bullet\left[b^{\prime} a^{\prime}\right]$ with a new label $c^{\prime}$ and $c \bullet\left[a^{\prime} b^{\prime}\right]$ and paste at $c$, we get $c^{\prime} c^{\prime} a^{\prime} b^{\prime} a^{\prime-1} b^{-1}$.

Step3. A subsequence of a labelling scheme is called $P$-form if it can be written as $\left(a_{1} a_{1}\right) \ldots\left(a_{m} a_{m}\right)$ or $T$-form if it can be written as $\left(a_{1} b_{1} a_{1}^{-1} b_{1}^{-1}\right) \ldots\left(a_{n} b_{n} a_{n}^{-1} b_{n}^{-1}\right)$. By the Classification Theorem, 


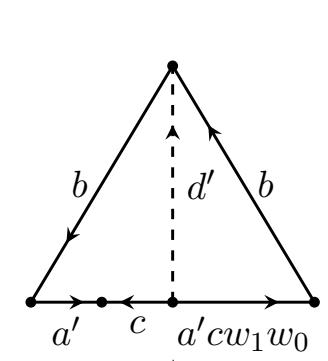

(1)

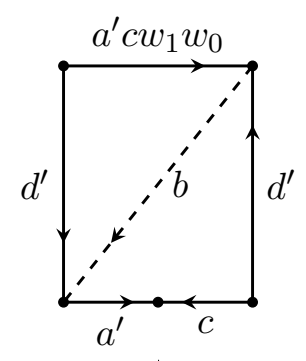

(2)

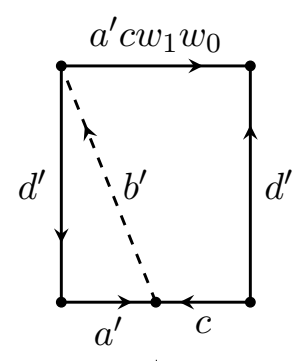

(3)

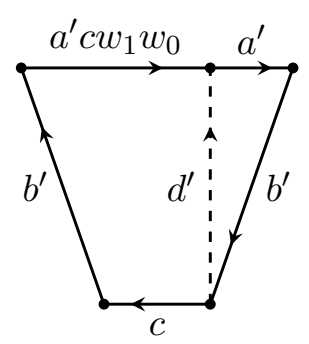

(4)

Figure 2.4:

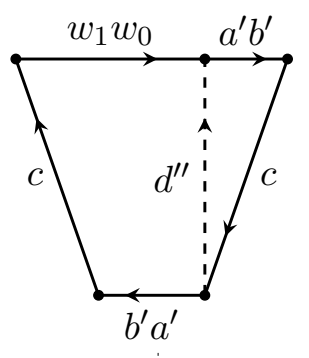

(1)

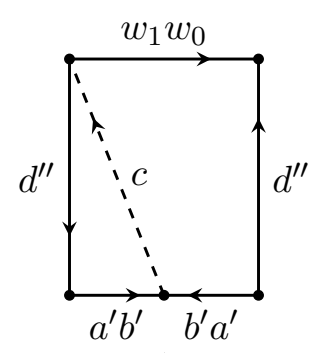

(2)

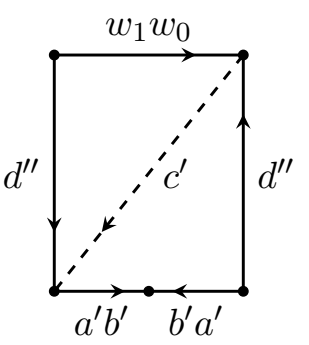

(3)

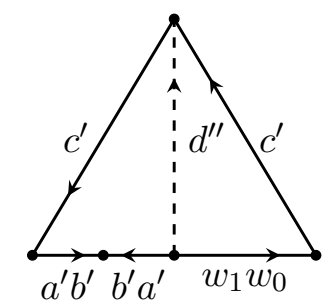

(4)

Figure 2.5:

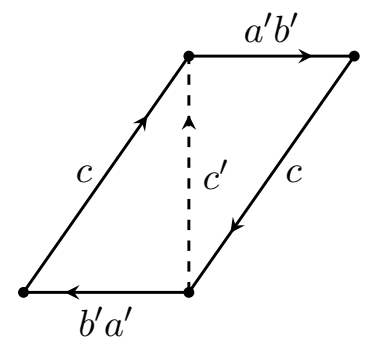

(1)

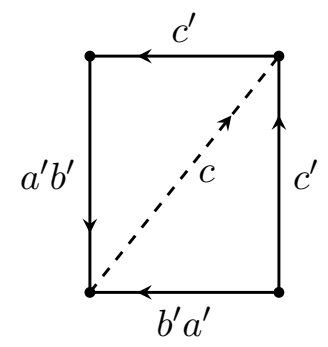

(2)

Figure 2.6: $w_{1} w_{0}=\emptyset$ 
if the surface $S$ is of T-form, the conclusion is obvious true. Let's consider the case of $P_{m}$ with $m \geq 3$, thus $S$ has the labelling scheme $w=\left(a_{1} a_{1}\right)\left(a_{2} a_{2}\right) \ldots\left(a_{m} a_{m}\right)$. Write $w \sim\left[w_{p}\right]\left[w_{t}\right]$ where $\left[w_{p}\right]$ is of P-form and $\left[w_{t}\right]$ is of T-form. Clearly $w$ has such a decomposition if we take $\left[w_{t}\right]=\emptyset$. Choose such a decomposition of $w$ such that $\left[w_{p}\right]$ has the minimum length. We claim that $\left[w_{p}\right]$ has length at most 4 , which corresponds to 1 or 2 cross-caps in the surface, so the conclusion holds. Suppose for contradiction that $w_{p}=\left(a_{1} a_{1}\right)\left(a_{2} a_{2}\right) \ldots\left(a_{k} a_{k}\right)$ with $k \geq 3$, then apply equation 2 by taking $w_{0}=\left(a_{1} a_{1}\right) \ldots\left(a_{k-3} a_{k-3}\right)$ and $w_{1}=w_{t}$, we have $w \sim\left[w_{0}\right]\left(a_{k} a_{k}\right)\left(a_{k-2} a_{k-1} a_{k-2}^{-1} a_{k-1}^{-1}\right)\left[w_{t}\right]$. Thus we get a decomposition with a shorter P-form $\left[w_{0}\right]\left(a_{k} a_{k}\right)$, a contradiction.

\subsubsection{Switch when cut-paste}

Let $P$ be a polygon representation with label scheme $w$. A pair of labels is called oriented pair (resp., opposite pair) if they have the same (resp., the opposite) orientation in $P$. For a cut-paste operation of $P$ with the cutting segment $L$. A pair of labels is called crossing pair if they are separated by $L$ in the polygon $P$, otherwise it is called side pair. For convenience reason, we view $L$, which will be assigned a pair of new label, a crossing pair.

Let $(G, \pi, S)$ be an embedded graph and $P$ be a polygon representation of $S$. The left and right-hand side of an edge $e$ of $G$ is exchanged when $e$ passes through an oriented pair while kept unchanged $e$ passes through an opposite pair. Thus the oriented pair (resp., the opposite pair) corresponds an odd number (resp., even number) of cross-caps. Now we can rewrite the natural signature of $(G, \pi, S)$ as follows: Define $\sigma(e)=-1$ if and only if $e$ passes through the oriented pairs odd times. We will use this definition in the proof of Theorem 2.1.3 and Theorem 2.1.4.

Proof of Theorem 2.1.3. Let $w$ and $w^{\prime}$ be two labelling schemes of $S$ such that $w^{\prime}$ is obtained from $w$ by a cut-paste. Let $\sigma$ and $\sigma^{\prime}$ be the corresponding natural signatures. We need to show that $\sigma \sim \sigma^{\prime}$.

(1) If the cut-paste operation is of type I, we want to show that $\sigma$ and $\sigma^{\prime}$ differ at an edge cut of $G$ : Let $P$ be the polygon representation of $S$. Assume that the cut-paste operation cuts along the segment $L=p_{i} p_{j}$ of $P$. Denote $P_{1}, P_{2}$ the two small regions of $P$ resulted by cutting along $L$. After some proper adjustments, we may assume that $V(G)$ have no intersection with $L$ as well as the boundary of $P$. Indeed, assume that $E(G)$ have no intersection with the vertices of $P$. Let $V_{i}$ be the vertices of $G$ contained in $P_{i}(i=1,2)$.

Now we claim that $\left(V_{1}, V_{2}\right)$ is the edge cut of $G$ where $\sigma$ differs from $\sigma^{\prime}$, hence $\sigma \sim \sigma^{\prime}$ :

By definition of natural signature and the analysis before the proof, $\sigma(e)<0$ if and only if $e$ passes through odd number of oriented pairs. For crossing pairs, the oriented pairs and the opposite pairs switch to the other after the cut-paste operation of type I, while for sided pairs, the oriented and the opposite cases kept unchanged. For any $e \in E\left(V_{1}, V_{2}\right)$, e must pass 
through the crossing pair odd times since its two end-vertices lie on different regions of $P$. Thus $\sigma(e)=-\sigma^{\prime}(e)$ since the signature of $e$ has changed odd times. For any $e \in G\left[V_{1}\right] \cup G\left[V_{2}\right]$, e passes through the crossing pair even times, thus $\sigma(e)=\sigma^{\prime}(e)$.

(2) If the cut-paste operation is of type II, then $\sigma=\sigma^{\prime}$ since the orientation of each pair (include $L$ ) does not change by the cut-paste of type II.

Definition 2.2.6. An glueing operation is to delete an adjacent opposite pair of labels in a label scheme.

Glueing operation, together with the cut-paste operation, are the only two operation involved in the proof of Classification Theorem. However, the case of glueing operation is quite simple for our topic. It doesn't change the natural signature at all since in the definition only oriented pairs are involved.

Now we are ready to prove Theorem 2.1.4

Proof of Theorem 2.1.4. For arbitrarily a signed graph $(G, \sigma)$, there exists a surface $S$ and an embedding of $G$ onto $S$ such that $\sigma$ is the corresponding natural signature. We want to find a polygon representation of the surface $S$.

Recall the construction in Proposition 2.2.1. Cut along all the cross-caps on $S$ and let $\mathcal{C}=\left\{C_{1}, \ldots, C_{t}\right\}$ be the boundaries of the cross-caps, which is a collection of circles on $S$. For each $C_{i} \in \mathcal{C}, C_{i}$ is divided by the edges of $G$ into an even number of segments. Insert a point at each segment of $C_{i}$. All these points are called inserted points, indicated by small circles in Figure 2.7. A path on $S$ is called connecting path if its two ends belong to the inserted points of $C_{i}$ and $C_{j}(i \neq j)$ and has no intersection with other $C_{k}(k \neq i, j)$. By the planarity of $S$, we can connect all the members of $\mathcal{C}$ together such that different connecting paths are interior-disjoint. Note that the connecting paths can have intersections with the edges of $G$ on the surface.

We can view this easily in a auxiliary graph with the vertex-set $\mathcal{C}$, and two circles are joined by an edge if and only if they are connected by a connecting path. Parallel edges are allowed. Thus the auxiliary graph is connected and we pick a spanning tree, which will give us the polygon representation: Cut along each connecting path and assign an opposite pair of labels on it. Each circle is divided into segments by the inserted points and assign every diametrical segments an orient pair of labels. All these circles together with the connecting paths (already doubled) give us a polygon representation by the structure of the spanning tree. Now $\sigma$ is the corresponding natural signature, thus $\sigma(e)=-1$ if and only if $e$ passes through the oriented pairs odd times.

By the Classification Theorem, we can convert the polygon representation of $S$ to the standard form Definition 2.2.5. In the process, only two operations are involved, namely cut-paste operation and glueing operation. Denoted by $\sigma^{\prime}$ the new natural signature. By Theorem 2.1.3 and the analysis before the proof, we have $\sigma \sim \sigma^{\prime}$ since both operations deduce the equivalent signatures. 


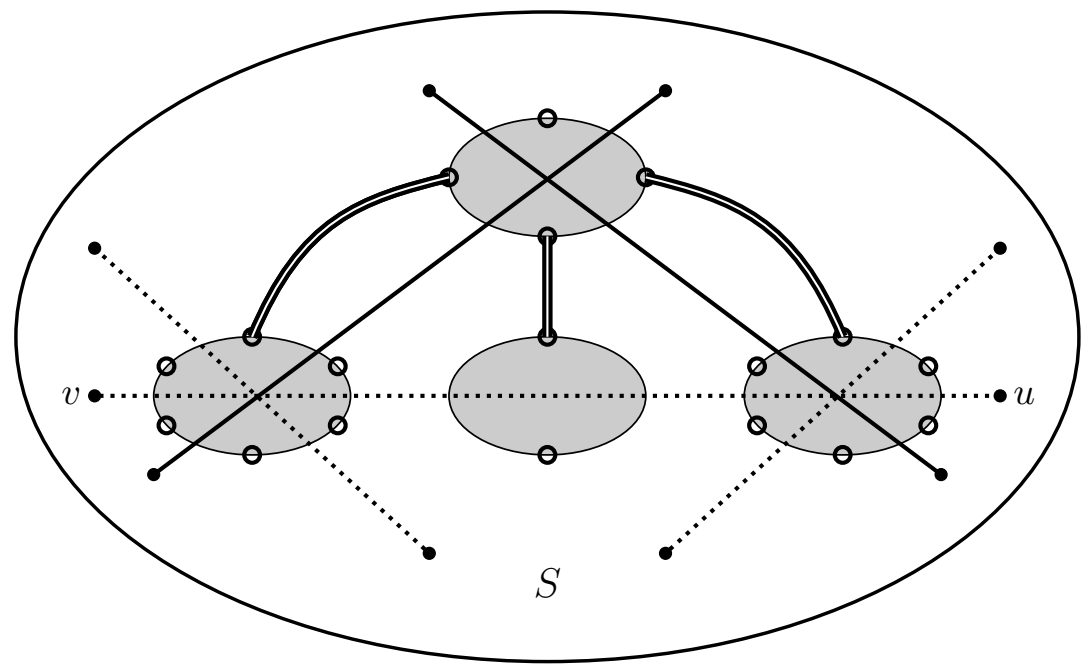

Figure 2.7: Polygon representation.

By Proposition 2.1.1, one can convert this standard form to a surface with at most 2 crosscaps and denote the new natural signature $\sigma^{\prime \prime}$. Applying Theorem 2.1.3 again, we get $\sigma^{\prime} \sim \sigma^{\prime \prime}$. By the hypothesis, $\left(G, \sigma^{\prime \prime}\right)$ admits a nowhere-zero 6 -flow. Hence $(G, \sigma)$ has a nowhere-zero 6-flow.

\subsection{Twin propeller graphs}

From now on, we just focus on the following special class of embedded graphs in Theorem 2.1.4 where the possible counterexamples can only occur: the embedded graphs whose surfaces have at most 2 cross-caps together with their natural signatures. We call this class of embedded signed graphs reduced class. In this section, we verify Bouchet's conjecture for a basic but important case in the reduced class, called twin propeller graphs.

Definition 2.3.1. Let $G$ is a signed graph with a positive circuit $C$. If all the negative edges occur as chords of $C$. Indeed, $E_{N}(G)$ has a partition $E_{N}(G)=E_{1} \cup E_{2}$ where $E_{1}=\left\{x_{i} w_{i}\right\}_{i=1}^{k}$ and $E_{2}=\left\{y_{j} z_{j}\right\}_{j=1}^{l}$ and end-vertices of $E_{N}$ lies on $C$ in the following cyclic order:

$$
x_{1}, \ldots, x_{k}, y_{1}, \ldots, y_{l}, z_{1}, \ldots, z_{l}, w_{1}, \ldots, w_{k} .
$$

Then we call $G$ a twin propeller graph, see Figure 2.8 .

Theorem 2.3.1. Every twin propeller graph admits a nowhere-zero 6-flow.

Similar to the proof of 6 -flow Theorem, the main method to prove Theorem 2.3.1 is to combine two particular 3 -flows together. 


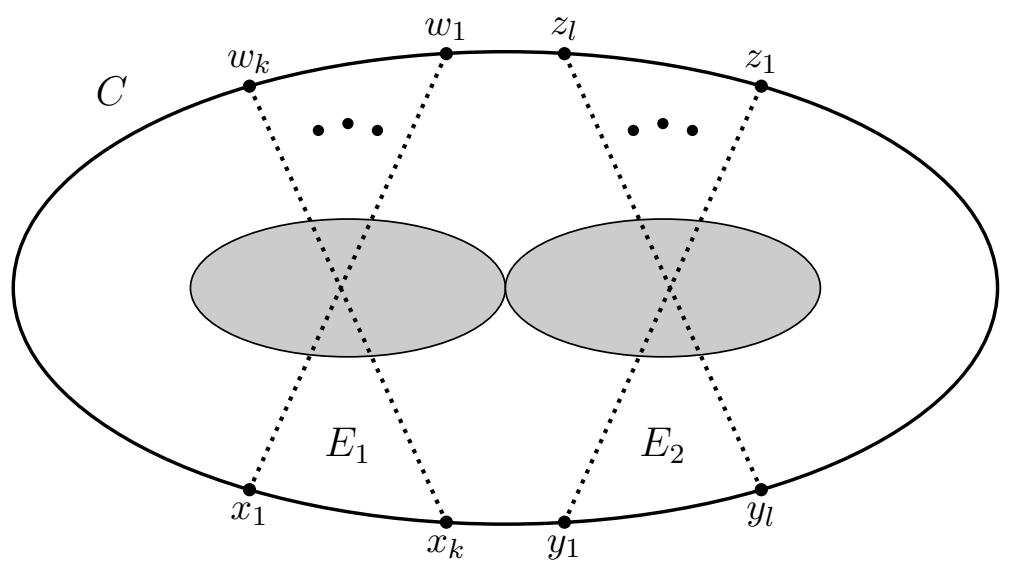

Figure 2.8: Twin propeller graph.

Proposition 2.3.1. Let $G$ be a signed graph and $f, h$ be 3 -flows of $G$. Suppose that supp $(f) \cup$ $\operatorname{supp}(g)=E(G)$ and $\left|\operatorname{supp}(f) \cap E_{ \pm 2}(g)\right| \leq 1$ on each component of supp $(g)$. Then $G$ has a $6-N Z F$.

Proof. Let $H_{1}, \ldots, H_{t}$ be the components of $\operatorname{supp}(g)$ and denote $g_{i}:=\left.g\right|_{E\left(H_{i}\right)}(1 \leq i \leq t)$. Then at least one of $2 f \pm g_{1}, \ldots, \pm g_{t}$ is a 6 -NZF of $G$.

To prove Theorem 2.3.1, we need some preparations, which are also powerful tools to deal with Bouchet's Conjecture. We next introduce some contractible configurations for Bouchet's 6-flow conjecture.

Lemma 2.3.2. (Lu, Luo and Zhang [21]) Let $k$ be a positive integer, and let $G$ be a graph with an orientation $\tau$ and admitting a $k-N Z F$. If a vertex $x$ of $G$ is of degree at most three and $g: \delta_{G}(x) \rightarrow\{ \pm 1, \ldots, \pm(k-1)\}$ satisfies $\partial g(x)=0$, then there is a $k$-NZF $(\tau, f)$ on $G$ such that $\left.f\right|_{\delta_{G}(x)}=g$.

Lemma 2.3.3. Let $G$ be a flow-admissible signed graph and $H$ be a subgraph of $G$ induced by a subset $X \subseteq V(G)$. If $\delta_{G}(X)+2\left|E_{N}(H)\right| \leq 3$, then $G$ admits a 6 -NZF if and only if so does $G / E\left(H-E_{N}(H)\right)$.

Proof. The "only if" part is obvious since all edges in $E(H)-E_{N}(H)$ are positive. We now prove the "if" part. Fix an arbitrary orientation $\tau$ of $G$. Let $H^{\prime}=H-E_{N}(H), G_{1}=G / E\left(H^{\prime}\right)$, and $\left(\tau_{1}, f_{1}\right)$ be a 6 -NZF of $G_{1}$, where $\tau_{1}$ is a restriction of $\tau$ on $H\left(G_{1}\right)$. To the end, we only need to extend $\left(\tau_{1}, f_{1}\right)$ to be a $6-\mathrm{NZF}$ of $G$.

If $H$ has a component $Q$ satisfying $\left|\delta_{G}(V(Q))\right|=0$ and $\left|E_{N}(Q)\right|=0$, then $Q$ is also a component of $G$. Since $G$ is flow-admissible, $Q$ is a bridgeless ordinary graph and thus admits a 
6-NZF by 6 -flow theorem. Hence assume that $H$ contains no such components. Since $G$ is flowadmissible and $\delta_{G}(X)+2\left|E_{N}(H)\right| \leq 3$, either $\left|E_{N}(H)\right|=0$ and $\left|\delta_{G}(X)\right| \in\{2,3\}$ or $\left|E_{N}(H)\right|=1$ and $\left|\delta_{G}(X)\right|=1$, moreover, $H$ is connected.

Let $H_{X}$ be the set of the half edges of each edge in $\delta_{G}(X) \cup E_{N}(H)$ whose end is in $X$. Then $\left|H_{X}\right|=\left|\delta_{G}(X)\right|+2\left|E_{N}(H)\right|=2$ or 3 . We add a new vertex $x$ to $H^{\prime}+H_{X}$ such that $x$ is the common end of all $h \in H_{X}$, and denote the new graph by $G_{2}$. Since $G$ is flow-admissible, $G_{2}$ is a bridgeless ordinary graph and thus admits a 6 -NZF by 6 -flow theorem.

Let $\tau_{2}$ be the restriction of $\tau$ on $H\left(G_{2}\right)$ and define $g(h)=f_{1}\left(e_{h}\right)$ for each $h \in H_{X}$. Note that $\tau_{2}(h)=\tau_{1}(h)$ for each $h \in H_{X}$ since $H_{X} \subseteq H\left(G_{1}\right)$. Since $\left(\tau_{1}, f_{1}\right)$ is a 6 -NZF of $G_{1}$, we have $\partial g(y)=-\partial f_{1}(y)=0$. By Lemma 2.3.2, there is a 6-NZF $\left(\tau_{2}, f_{2}\right)$ of $G_{2}$ such that for every $h \in H_{x}, f_{2}(h)=g(h)=f_{1}\left(e_{h}\right)$, and thus $\left(\tau_{1}, f_{1}\right)$ can be extended to a 6 -NZF of $G$.

In general, the existence of $k-\mathrm{NZF}$ and $\mathbb{Z}_{k}-\mathrm{NZF}$ are not equivalent for all signed graphs. However, we have the following equivalent relation when we restrict our cases on the signed graphs with small negativeness and $k=3$.

Lemma 2.3.4. Every signed graph with at most two negative edge admits a 3-NZF if and only if it admits a $\mathbb{Z}_{3}-N Z F$.

Proof. We only need to prove the sufficiency. Let $G$ be a signed graph such that

(1) $G$ admits a $\mathbb{Z}_{3}$-NZF $(\tau, f)$, but does not admit 3 -NZFs;

(2) subject to (1), $\sum_{v \in V(G)}\left|d_{G}(x)-3\right|$ is as small as possible.

Then $G$ is flow-admissible and is of negativeness two. Further, assume that $G$ is connected.

Claim 2.3.1. $G$ is cubic.

Proof of Claim 2.3.1. By the choice of $G$, it is trivial that every vertex of $G$ is of degree at most 3. Suppose that $x$ is a vertex of $G$ with $d_{G}(x) \geq 4$. Pick arbitrarily two edges $e$ and $e^{\prime}$ from $E_{G}(x)$, and let $G_{\left[x,\left\{e, e^{\prime}\right\}\right]}$ be the signed graph obtained from $G$ by adding a new vertex $x^{\prime}$ and changing the end $x$ of $e$ and $e^{\prime}$ to be $x^{\prime}$. If $\tau\left(h_{e}^{x}\right) f(e)+\tau\left(h_{e^{\prime}}^{x}\right) f\left(e^{\prime}\right) \equiv 0(\bmod 3)$, then let $G^{\prime}$ be the suppressed graph of $G_{\left[x,\left\{e, e^{\prime}\right\}\right]}$. If $\tau\left(h_{e}^{x}\right) f(e)+\tau\left(h_{e^{\prime}}^{x}\right) f\left(e^{\prime}\right) \not \equiv 0(\bmod 3)$, then let $G^{\prime}$ be the signed graph obtained from $G_{\left[x,\left\{e, e^{\prime}\right\}\right]}$ by adding a new positive edge $x x^{\prime}$. In both cases, $G^{\prime}$ admits a $\mathbb{Z}_{3}$-NZF and satisfies $\sum_{v \in V\left(G^{\prime}\right)}\left|d_{G^{\prime}}(x)-3\right|<\sum_{v \in V(G)}\left|d_{G}(x)-3\right|$. By the choice of $G$ again, $G^{\prime}$ admits a $3-\mathrm{NZF}$, and so does $G$. This contradicts (1).

Let $E_{N}(G)=\left\{e_{1}, e_{2}\right\}, e_{1}=x_{1} y_{1}$ and $e_{2}=x_{2} y_{2}$. We construct a new graph $G^{*}$ from $G$ as follows: insert a new vertex $z_{i}$ for $i=1,2$ of degree 2 into $e_{i}$, and add a new positive edge $e^{*}=z_{1} z_{2}$.

Claim 2.3.2. $G^{*}$ is unsigned, cubic, 2-edge-connected and bipartite. 
Proof of Claim 2.3.2. It is obvious that $G^{*}$ is unsigned and cubic since $E_{N}(G)=\left\{e_{1}, e_{2}\right\}$ and $G$ is cubic by Claim 2.3.1. Since a connected cubic graph is 2-edge-connected and bipartite if and only if it admits $3-\mathrm{NZF}$, we only need to prove that $G^{*}$ admits a 3 -NZF below.

Note that $E\left(G^{*}\right)=\left(E(G) \backslash\left\{e_{1}, e_{2}\right\}\right) \cup\left\{z_{1} x_{1}, z_{1} y_{1}, z_{2} x_{2}, z_{2} y_{2}, e^{*}\right\}$. For any $e=x y \in E\left(G^{*}\right)$, we orient $e$ away from $x$ if $\tau\left(h_{e}^{x}\right)=1$ or $x=z_{1}$ and $y=z_{2}$, and toward $x$ otherwise. Denote this orientation of $G^{*}$ by $\tau^{*}$. Define a mapping $f^{*}: E\left(G^{*}\right) \rightarrow \mathbb{Z}_{3}$ by

$$
f^{*}(e)= \begin{cases}f(e) & \text { if } e \in E(G) \backslash\left\{e_{1}, e_{2}\right\} \\ f\left(e_{1}\right) & \text { if } e \in\left\{z_{1} x_{1}, z_{1} y_{1}\right\} \\ f\left(e_{2}\right) & \text { if } e \in\left\{z_{2} x_{2}, z_{2} y_{2}\right\} \\ f\left(e_{1}\right) \cdot 2 \tau\left(h_{e_{1}}^{x_{1}}\right) & \text { if } e=e^{*}\end{cases}
$$

Since $E_{N}(G)=\left\{e_{1}, e_{2}\right\}$ and $(\tau, f)$ is a $\mathbb{Z}_{3}$-NZF of $G,\left|f\left(e_{1}\right)\right|=\left|f\left(e_{2}\right)\right|$, and thus it is not difficult to check that $\left(\tau^{*}, f^{*}\right)$ is a $\mathbb{Z}_{3}-\mathrm{NZF}$ of $G^{*}$.

By Petersen theorem and Claim 2.3.2, $G^{*}$ has a 1-factor $M$ containing $e^{*}$, furthermore, admits a 3 -NZF $\left(\tau_{1}, f_{1}\right)$ such that $\left|f\left(e^{*}\right)\right|=2$. By the construction of $G^{*}, G$ is the suppressed graph $G^{*}-e^{*}$, and the restriction of $\left(\tau_{1}, f_{1}\right)$ on $E(G)$ is a 3 -NZF of $G$, a contradiction to the assumption.

The first 3-flow in Proposition 2.3.1 is constructed by $\Phi_{2}$-operations, which Seymour introduced to prove the 6 -flow theorem for ordinary graphs. Actually it can be generalized to signed graphs.

$$
\Phi_{k} \text { : add a balanced circuit or a barbell } C \text { into } G \text { if }|E(C) \backslash E(G)| \leq k \text {. }
$$

For a subgraph $H$ of $G$, denote by $\langle H\rangle_{k}$ the maximum subgraph of $G$ obtained from $H$ via $\Phi_{k}$-operations.

Lemma 2.3.5. (Seymour) Let $G$ be a 3-edge-connected graph and $v \in V(G)$. Then there is an even subgraph $H$ of $G-v$ such that $\langle H\rangle_{2}=G$.

With a similar argument to the proof of Seymour's 6-flow theorem, Zýka obtained the following result.

Lemma 2.3.6. (Zýka [39]) Let $G$ be a signed graph and $H$ be a subgraph of $G$. If $\langle H\rangle_{2}=G$, then $G$ admits a $\mathbb{Z}_{3}$-flow $(\tau, f)$ such that $E(G) \backslash E(H) \subseteq \operatorname{supp}(f)$.

The second 3-flow in Proposition 2.3.1 will be constructed at the local structure around the circuit $C$. Let $C$ be a circuit of $G$ with even length, the pace of a chord $e=x y$ of $C$ is the length of the path $x C y$ of $G$. 
Proposition 2.3.2. Let $G$ be a cubic signed graph with a all-positive Hamilton circuit $C$. Suppose each positive chord of $C$ has odd pace and each negative chord has even pace. Then $G$ admits a 3 -NZF $g$ such that $E_{ \pm 1}(g)=E(C)$ and $E_{ \pm 2}(g)=E(G) \backslash E(C)$

Proof. Orient the edges of $C$ alternatively along the circuit $C$ and set all these edges with value 1. Since each positive chord has even pace, the boundaries of its two end-vertices have different signs. Orient it towards the vertex with positive boundary and assign it value 2. Similar for the negative chords. We get the required flow.

Proof of Theorem 2.3.1. Let $G$ be a twin propeller signed graph and $E_{N}(G)=E_{1} \cup E_{2}$ be the partition of the negative edges in Definition 2.3.1. Denote $G^{+}:=G-E_{N}(G)$ and $G^{\prime}:=$ $G^{+} / E(C)$. By Lemma 2.3.3, $G^{\prime}$ is 3-edge connected. Hence there exists an even subgraph $K$ in $G^{+}-v_{C}$ such that $\langle K\rangle_{2}=G^{\prime}$ by Lemma 2.3 .5 where $v_{C}$ is the contracted vertex corresponding to $C$. Thus $\langle K \cup C\rangle_{2}=G^{+}$in the ordinary graph $G^{+}$. By Lemma 2.3.6, $G^{+}$admits a $\mathbb{Z}_{3}$-flow $f_{1}$ such that $\operatorname{supp}\left(f_{1}\right) \supseteq E\left(G^{+}\right)-E(C \cup K)$. We have the following three cases to analyse according to the parity of $E_{1}$ and $E_{2}$. Pick $e_{i} \in E_{i}$ if $E_{i} \neq \emptyset$ for $i=1,2$.

Case 1 Both $\left|E_{1}\right|$ and $\left|E_{2}\right|$ are even. Denote $H:=C+E_{N}$. Then $H$ satisfies the conditions of Proposition 2.3.2 by the analysis above. Thus $H$ admits a 3-NZF $f_{2}$ such that $E_{ \pm 1}\left(f_{2}\right)=E(C)$ and $E_{ \pm 2}\left(f_{2}\right)=E_{N}(G)$. Let $\tilde{f}_{2}$ be the 3-flow of $G$ obtained from $f_{2}$ by adding a 2-NZF on $K$ and $\tilde{f}_{1}$ be the 3 -flow having the same support with $f_{1}$ by Lemma 2.3.4. Clearly $\tilde{f}_{1}$ and $\tilde{f}_{2}$ satisfies the conditions of Proposition 2.3.1, thus $G$ admits a $6-\mathrm{NZF}$.

Case 2 One of $\left|E_{1}\right|,\left|E_{2}\right|$ is odd, say $\left|E_{1}\right|$. Let $H^{\prime}:=C+E_{N}-\left\{e_{1}\right\}$. Again by Proposition 2.3.2, $H^{\prime}$ admits a 3 -NZF $f_{2}^{\prime}$, which can be extend to $K$, denoted by $\tilde{f}_{2}^{\prime}$. Since $\langle K \cup C\rangle_{2}=G^{+}$, we can extend this $\phi_{2}$ sequence by adding a balance circuit containing $\left\{e_{1}, e_{2}\right\}$ at the final step. Therefore $\langle K \cup C\rangle_{2}=G^{+}+\left\{e_{1}, e_{2}\right\}$. Then by Lemma 2.3.6, there exists a $\mathbb{Z}_{3}$-flow $f_{1}^{\prime}$ such that $\operatorname{supp}\left(f_{1}^{\prime}\right) \supseteq E\left(G^{+}\right)+\left\{e_{1}, e_{2}\right\}-E(C \cup K)$. Denoted by $\tilde{f}_{1}^{\prime}$ the 3-flow having the same support with $f_{1}^{\prime}$. Again, $\tilde{f}_{1}^{\prime}$ and $\tilde{f}_{2}^{\prime}$ satisfies Proposition 2.3.1, done.

Case 3 Both of $\left|E_{1}\right|,\left|E_{2}\right|$ are odd. Let $H^{\prime \prime}:=C+E_{N}-\left\{e_{1}, e_{2}\right\}$ and $f_{2}^{\prime \prime}$ be a $3-\mathrm{NZF}$ of $H^{\prime \prime}$ by Proposition 2.3.2. Denote by $\tilde{f}_{2}^{\prime \prime}$ an extension of $f_{2}^{\prime \prime}$ to $K$. Similar to case 2, there exists a 3-flow $\tilde{f}_{1}^{\prime \prime}$ such that $\operatorname{supp}\left(f_{1}^{\prime \prime}\right) \supseteq E\left(G^{+}\right)+\left\{e_{1}, e_{2}\right\}-E(C \cup K)$. By Proposition 2.3.1 again, we can combine $\tilde{f_{1}^{\prime \prime}}$ and $\tilde{f}_{2}^{\prime \prime}$ together to get a $6-\mathrm{NZF}$ of $G$. 


\section{Chapter 3}

\section{1-FLOW}

\subsection{Introduction}

In this Chapter, we provide a best partial result to Bouchet's Conjecture.

In 1983, Bouchet [5] proposed a flow conjecture that every flow-admissible signed graph admits a nowhere-zero 6-flow. Bouchet [5] himself proved that such signed graphs admit nowherezero 216-flows; Zýka [39] proved that such signed graphs admit nowhere-zero 30-flows. In this Chapter, we prove the following result.

Theorem 3.1.1. Every flow-admissible signed graph admits a nowhere-zero 11-flow.

In fact, we prove a stronger and very structural result as follows, and Theorem 3.1.1 is an immediate corollary.

Theorem 3.1.2. Every flow-admissible signed graph $G$ admits a 3 -flow $f_{1}$ and a 5 -flow $f_{2}$ such that $f=3 f_{1}+f_{2}$ is a nowhere-zero 11-flow, $|f(e)| \neq 9$ for each edge $e$, and $|f(e)|=10$ only if $e \in B\left(\operatorname{supp}\left(f_{1}\right)\right) \cap B\left(\operatorname{supp}\left(f_{2}\right)\right)$, where $B\left(\operatorname{supp}\left(f_{i}\right)\right)$ is the set of all bridges of the subgraph induced by the edges of $\operatorname{supp}\left(f_{i}\right)(i=1,2)$.

Theorem 3.1.2 may suggest an approach to further reduce 11-flows to 9-flows.

The main approach to prove the 11-flow theorem is the following result, which, we believe, will be a powerful tool in the study of integer flows of signed graphs, in particular to resolve Bouchet's 6-flow conjecture.

Theorem 3.1.3. Every flow-admissible signed graph admits a balanced nowhere-zero $\mathbb{Z}_{2} \times \mathbb{Z}_{3}$ flow.

A $\mathbb{Z}_{2} \times \mathbb{Z}_{3}$-flow $\left(f_{1}, f_{2}\right)$ is called balanced if $\operatorname{supp}\left(f_{1}\right)$ contains an even number of negative edges. 
The rest of the chapter is organized as follows: Basic notations and definitions will be introduced in Section 3.2. Section 3.3 will discuss the conversion of modulo flows into integer flows. In particular a new result to convert a modulo 3 -flow to an integer 5 -flow will be introduced and its proof will be presented in Section 3.5. The proofs of Theorems 3.1.2 and 3.1.3 will be presented in Sections 3.4 and 3.6, respectively.

\subsection{Signed graphs, switch operations, and flows}

Let $G$ be a graph. The degree of $v \in V(G)$ is the number of edges incident with $v$, where each loop is counted twice. A $d$-vertex is a vertex with degree $d$. Let $V_{d}(G)$ be the set of $d$-vertices in $G$. The maximum degree of $G$ is denoted by $\Delta(G)$. We use $B(G)$ to denote the set of cut-edges of $G$.

A signed graph $(G, \sigma)$ is a graph $G$ together with a signature $\sigma: E(G) \rightarrow\{-1,1\}$. More definitions about signed graphs, such as equivalence, orientation, are defined in Chapter 2. Moreover, we define the negativeness of $G$ by $\epsilon(G)=\min \left\{\left|E_{N}\left(G, \sigma^{\prime}\right)\right|: \sigma^{\prime}\right.$ is equivalent to $\left.\sigma\right\}$. A signed graph is balanced if its negativeness is 0 . That is, it is equivalent to a graph without negative edges. For a subgraph $G^{\prime}$ of $G$, denote $\sigma\left(G^{\prime}\right)=\prod_{e \in E\left(G^{\prime}\right)} \sigma(e)$. For convenience, the signature $\sigma$ is usually omitted if no confusion arises or is written as $\sigma_{G}$ if it needs to emphasize $G$.

Recall the integer flows of signed graphs we introduced in Chapter 2, it is so basic and important, and we just repeat it here.

Definition 3.2.1. Assume that $G$ is a signed graph associated with an orientation $\tau$. Let $A$ be an abelian group and $f: E(G) \rightarrow A$ be a mapping. The boundary of $f$ at a vertex $v$ is defined as

$$
\partial f(v)=\sum_{h \in H_{G}(v)} \tau(h) f\left(e_{h}\right)
$$

The pair $(\tau, f)$ (or to simplify, $f$ ) is an A-flow of $G$ if $\partial f(v)=0$ for each $v \in V(G)$, and is an (integer) $k$-flow if it is a $\mathbb{Z}$-flow and $|f(e)|<k$ for each $e \in E(G)$.

Let $f$ be a flow of a signed graph $G$. The support of $f$, denoted by $\operatorname{supp}(f)$, is the set of edges $e$ with $f(e) \neq 0$. The flow $f$ is nowhere-zero if $\operatorname{supp}(f)=E(G)$. For convenience, we abbreviate the notions of nowhere-zero $A$-flow and nowhere-zero $k$-flow as $A$-NZF and $k$-NZF, respectively. Observe that $G$ admits an $A$-NZF (resp., a $k$-NZF) under an orientation $\tau$ if and only if it admits an $A$-NZF (resp., a $k$-NZF) under any orientation $\tau^{\prime}$. A $\mathbb{Z}_{k}$-flow is also called a modulo $k$-flow. For an integer flow $f$ of $G$ and a positive integer $t$, let $E_{f= \pm t}:=\{e \in E(G):|f(e)|=t\}$.

A signed graph $G$ is flow-admissible if it admits a $k$-NZF for some positive integer $k$. Bouchet [5] characterized all flow-admissible signed graphs as follows. 
Proposition 3.2.1. ( [5]) A connected signed graph $G$ is flow-admissible if and only if $\epsilon(G) \neq 1$ and there is no cut-edge $b$ such that $G-b$ has a balanced component.

\subsection{Modulo flows on signed graphs}

Just like in the study of flows of ordinary graphs and as Theorem 3.1.3 indicates, the key to make further improvement and to eventually solve Bouchet's 6-flow conjecture is to further study how to convert modulo 2-flows and modulo 3 -flows into integer flows. The following lemma converts a modulo 2-flow into an integer 3 -flow.

Lemma 3.3.1 ( [6]). If a signed graph is connected and admits a $\mathbb{Z}_{2}$-flow $f_{1}$ such that $\operatorname{supp}\left(f_{1}\right)$ contains an even number of negative edges, then it also admits a 3 -flow $f_{2}$ such that $\operatorname{supp}\left(f_{1}\right) \subseteq$ $\operatorname{supp}\left(f_{2}\right)$ and $\left|f_{2}(e)\right|=2$ if and only if $e \in B\left(\operatorname{supp}\left(f_{2}\right)\right)$.

Remark 1. In Lemma 3.3.1 the conclusion " $f_{2}(e) \mid=2$ if and only if $e \in B\left(\operatorname{supp}\left(f_{2}\right)\right)$ " is not listed in Theorem 1.5 of [6]. However this fact is implicit and follows from the basic property of flows of signed graphs: the flow value of each cut-edge must be even.

In this paper, we will show that one can convert a $\mathbb{Z}_{3}$-NZF to a very special 5 -NZF.

Theorem 3.3.2. Let $G$ be a signed graph admitting a $\mathbb{Z}_{3}-N Z F$. Then $G$ admits a 5 -NZF $g$ such that $E_{g= \pm 3}=\emptyset$ and $E_{g= \pm 4} \subseteq B(G)$.

Theorem 3.3.2 is also a key tool in the proof of the 11-theorem and its proof will be presented in Section 3.5.

Remark 2. Theorem 3.3.2 is sharp in the sense that there is an infinite family of signed graphs that admits a $\mathbb{Z}_{3}-N Z F$ but does not admit a 4-NZF. For example, the signed graph obtained from a tree in which each vertex is of degree one or three by adding a negative loop at each vertex of degree one. An illustration is shown in Fig. 3.1.

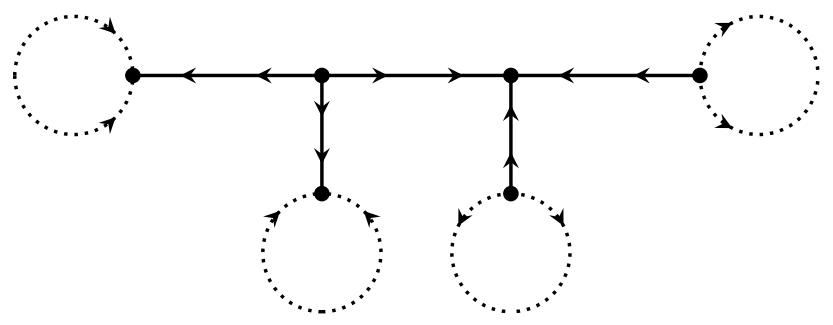

Figure 3.1: A signed graph admitting a $\mathbb{Z}_{3}-\mathrm{NZF}$ with all edges assigned with 1 , but no 4-NZF. 


\subsection{Proof of the 11-flow theorem}

Now we are ready to prove Theorem 3.1.2, assuming Theorems 3.1.3 and 3.3.2.

Proof of Theorem 3.1.2. Let $G$ be a connected flow-admissible signed graph. By Theorem 3.1.3, $G$ admits a balanced $\mathbb{Z}_{2} \times \mathbb{Z}_{3}$-NZF $\left(g_{1}, g_{2}\right)$. By Lemma 3.3.1, $G$ admits a 3 -flow $f_{1}$ such that $\operatorname{supp}\left(g_{1}\right) \subseteq \operatorname{supp}\left(f_{1}\right)$ and $\left|f_{1}(e)\right|=2$ if and only if $e \in B\left(\operatorname{supp}\left(f_{1}\right)\right)$.

By Theorem 3.3.2, $G$ admits a 5 -flow $f_{2}$ such that $\operatorname{supp}\left(f_{2}\right)=\operatorname{supp}\left(g_{2}\right)$ and

$$
E_{f_{2}= \pm 3}=\emptyset
$$

Since $\left(g_{1}, g_{2}\right)$ is a $\mathbb{Z}_{2} \times \mathbb{Z}_{3}$-NZF of $G$,

$$
\operatorname{supp}\left(f_{1}\right) \cup \operatorname{supp}\left(f_{2}\right)=\operatorname{supp}\left(g_{1}\right) \cup \operatorname{supp}\left(g_{2}\right)=E(G) .
$$

We are to show that $f=3 f_{1}+f_{2}$ is a nowhere-zero 11-flow described in the theorem. Since $\left|f_{1}(e)\right| \leq 2$ and $\left|f_{2}(e)\right| \leq 4$, we have

$$
|f(e)|=\left|\left(3 f_{1}+f_{2}\right)(e)\right| \leq 3\left|f_{1}(e)\right|+\left|f_{2}(e)\right| \leq 10 \quad \forall e \in E(G) .
$$

Furthermore, by applying Equations (3.1) and (3.2),

$$
3 f_{1}(e)+f_{2}(e) \neq 0, \pm 9 \quad \forall e \in E(G) .
$$

If $|f(e)|=10$ for some edge $e \in E(G)$, then $\left|f_{1}(e)\right|=2$ and $\left|f_{2}(e)\right|=4$. Thus, by Lemmas 3.3.1 and 3.3.2 again, the edge $e \in B\left(\operatorname{supp}\left(f_{1}\right)\right) \cap B\left(\operatorname{supp}\left(f_{2}\right)\right)$ and hence $f=3 f_{1}+f_{2}$ is the 11-NZF described in Theorem 3.1.2.

\subsection{Proof of Theorem 3.3.2}

As the preparation of the proof of Theorem 3.3.2, we first need some necessary lemmas.

The first lemma is a stronger form of the famous Petersen's theorem, and here we omit its proof (see Exercise 16.4.8 in [4]).

Lemma 3.5.1. Let $G$ be a bridgeless cubic graph and $e_{0} \in E(G)$. Then $G$ has two perfect matchings $M_{1}$ and $M_{2}$ such that $e_{0} \in M_{1}$ and $e_{0} \notin M_{2}$.

We also need a splitting lemma due to Fleischner [10].

Let $G$ be a graph and $v$ be a vertex. If $F \subset \delta_{G}(v)$, we denote by $G_{[v ; F]}$ the graph obtained from $G$ by splitting the edges of $F$ away from $v$. That is, adding a new vertex $v^{*}$ and changing the common end of edges in $F$ from $v$ to $v^{*}$. 
Lemma 3.5.2. ( [10]) Let $G$ be a bridgeless graph and $v$ be a vertex. If $d_{G}(v) \geq 4$ and $e_{0}, e_{1}, e_{2} \in$ $\delta_{G}(v)$ are chosen in a way that $e_{0}$ and $e_{2}$ are in different blocks when $v$ is a cut-vertex, then either $G_{\left[v ;\left\{e_{0}, e_{1}\right\}\right]}$ or $G_{\left[v ;\left\{e_{0}, e_{2}\right\}\right]}$ is bridgeless. Furthermore, $G_{\left[v ;\left\{e_{0}, e_{2}\right\}\right]}$ is bridgeless if $v$ is a cut-vertex.

Let $G$ be a signed graph. A path $P$ in $G$ is called a subdivided edge of $G$ if every internal vertex of $P$ is a 2-vertex. The suppressed graph of $G$, denoted by $\bar{G}$, is the signed graph obtained from $G$ by replacing each maximal subdivided edge $P$ with a single edge $e$ and assigning $\sigma(e)=\sigma(P)$.

The following result is proved in [33] which gives a sufficient condition when a modulo 3-flow and an integer 3-flow are equivalent for signed graphs.

Lemma 3.5.3 ( [33]). Let $G$ be a bridgeless signed graph. If $G$ admits a $\mathbb{Z}_{3}-N Z F$, then it also admits a $3-N Z F$.

Lemma 3.5.3 is strengthened in the following lemma, which will serve as the induction base in the proof of Theorem 3.3.2.

Lemma 3.5.4. Let $G$ be a bridgeless signed graph admitting a $\mathbb{Z}_{3}-N Z F$. Then for any $e_{0} \in E(G)$ and for any $i \in\{1,2\}, G$ admits a $3-N Z F$ such that $e_{0}$ has the flow value $i$.

Proof. Let $G$ be a counterexample with $\beta(G):=\sum_{v \in V(G)}\left|d_{G}(v)-2.5\right|$ minimum. Since $G$ admits a $\mathbb{Z}_{3}$-NZF, there is an orientation $\tau$ of $G$ such that for each $v \in V(G)$,

$$
\partial \tau(v):=\sum_{h \in H_{G}(v)} \tau(h) \equiv 0 \quad(\bmod 3) .
$$

We claim $\Delta(G) \leq 3$. Suppose to the contrary that $G$ has a vertex $v$ with $d_{G}(v) \geq 4$. By Lemma 3.5.2, we can split a pair of edges $\left\{e_{1}, e_{2}\right\}$ from $v$ such that the new signed graph $G^{\prime}=G_{\left[v ;\left\{e_{1}, e_{2}\right\}\right]}$ is still bridgeless. In $G^{\prime}$, we consider $\tau$ as an orientation on $E\left(G^{\prime}\right)$ and denote the common end of $e_{1}$ and $e_{2}$ by $v^{*}$. If $\partial \tau\left(v^{*}\right)=0$, then $\beta\left(G^{\prime}\right)<\beta(G)$ and by Eq. (3.3), $\partial \tau(u) \equiv 0$ $(\bmod 3)$ for each $u \in V\left(G^{\prime}\right)$, a contradiction to the minimality of $\beta(G)$. If $\partial \tau\left(v^{*}\right) \neq 0$, then we further add a positive edge $v v^{*}$ to $G^{\prime}$ and denote the resulting signed graph by $G^{\prime \prime}$. Let $\tau^{\prime \prime}$ be the orientation of $G^{\prime \prime}$ obtained from $\tau$ by assigning $v v^{*}$ with a direction such that $\partial \tau^{\prime \prime}\left(v^{*}\right) \equiv 0$ $(\bmod 3)$. Then by Eq. $(3.3), \partial \tau^{\prime \prime}(u) \equiv 0(\bmod 3)$ for each $u \in V\left(G^{\prime \prime}\right)$. Since $\beta\left(G^{\prime \prime}\right)<\beta(G)$, we obtain a contradiction to the minimality of $\beta(G)$ again. Therefore $\Delta(G) \leq 3$.

Since $G$ is bridgeless, every vertex of $G$ is of degree 2 or 3 . Note that the existence of the desired 3-flows is preserved under the suppressing operation. Then the suppressed signed graph $\bar{G}$ of $G$ is also a counterexample, and $\beta(\bar{G})<\beta(G)$ when $G$ has some 2 -vertices. Therefore $G$ is cubic by the minimality of $\beta(G)$.

Since $G$ is cubic, by Eq. (3.3), either $\partial \tau(v)=d_{G}(v)$ or $\partial \tau(v)=-d_{G}(v)$ for each $v \in V(G)$. By Lemma 3.5.1, we can choose two perfect matchings $M_{1}$ and $M_{2}$ such that $e_{0} \notin M_{1}$ and $e_{0} \in M_{2}$. For $i=1,2$, let $\tau_{i}$ be the orientation of $G$ obtained from $\tau$ by reversing the directions 
of all edges of $M_{i}$, and define a mapping $f_{i}: E(G) \rightarrow\{1,2\}$ by setting $f_{i}(e)=2$ if $e \in M_{i}$ and $f_{i}(e)=1$ if $e \notin M_{i}$. Then $f_{1}$ and $f_{2}$ are two desired nowhere-zero 3-flows of $G$ under $\tau_{1}$ and $\tau_{2}$, respectively, a contradiction.

Now we are ready to complete the proof of Theorem 3.3.2.

Proof of Theorem 3.3.2. We will prove by induction on $t=|B(G)|$, the number of cut-edges in $G$. If $t=0$, then $G$ is bridgeless and it is a direct corollary of Lemma 3.5.4. This establishes the base of the induction.

Assume $t>0$. Let $e=v_{1} v_{2}$ be a cut-edge in $B(G)$ such that one component, say $B_{1}$, of $G-e$ is minimal. Let $B_{2}$ be the other component of $G-e$. We may assume the bridge $e$ is a positive edge (by possibly some switching operations). Since $G$ admits a $\mathbb{Z}_{3}$-NZF, $\delta(G) \geq 2$. Thus $B_{1}$ is bridgeless and nontrivial. WLOG assume $v_{i} \in B_{i}(i=1,2)$. Let $B_{i}^{\prime}$ be the graph obtained from $B_{i}$ by adding a negative loop $e_{i}$ at $v_{i}$. Then $B_{i}^{\prime}$ admits a $\mathbb{Z}_{3}$-NZF since $G$ admits a $\mathbb{Z}_{3}$-NZF. By induction hypothesis, $B_{2}^{\prime}$ admits a 5 -NZF $g_{2}$ with $g_{2}\left(e_{2}\right)=a \in\{1,2\}$. By Lemma 3.5.4, $B_{1}^{\prime}$ admits a 3 -NZF $g_{1}$ such that $g_{1}\left(e_{1}\right)=a$. Hence we can extend $g_{1}$ and $g_{2}$ to a 5 -NZF $g$ of $G$ by setting $g(e)=2 a$ with appropriate orientation of $e$. Clearly $g$ is a desired 5-NZF of $G$.

\subsection{Proof of Theorem 3.1.3}

In this section, we will complete the proof of Theorem 3.1.3, which is divided into two steps: first to reduce it from general flow-admissible signed graphs to cubic shrubberies (see Lemma 3.6.5); and then prove that every cubic shrubbery admits a balanced $\mathbb{Z}_{2} \times \mathbb{Z}_{3}$-NZF by showing a stronger result (see Lemma 3.6.10).

We first need some terminology and notations. Let $G$ be a graph. For an edge $e \in E(G)$, contracting $e$ is done by deleting $e$ and then (if $e$ is not a loop) identifying its ends. Note that all resulting loops generated from the parallel edges of $e$ are kept. For $S \subseteq E(G)$, we use $G / S$ to denote the resulting graph obtained from $G$ by contracting all edges in $S$.

For a path $P$, let $\operatorname{End}(P)$ and $\operatorname{Int}(P)$ be the sets of the ends and internal vertices of $P$, respectively. For $U_{1}, U_{2} \subseteq V(G)$, a $\left(U_{1}, U_{2}\right)$-path is a path $P$ satisfying $\left|\operatorname{End}(P) \cap U_{i}\right|=1$ and $\operatorname{Int}(P) \cap U_{i}=\emptyset$ for $i=1,2$; if $G_{1}$ and $G_{2}$ are subgraphs of $G$, we write $\left(G_{1}, G_{2}\right)$-path instead of $\left(V\left(G_{1}\right), V\left(G_{2}\right)\right)$-path. Let $C=v_{1} \cdots v_{r} v_{1}$ be a circuit. A segment of $C$ is the path $v_{i} v_{i+1} \cdots v_{j-1} v_{j}(\bmod r)$ contained in $C$ and is denoted by $v_{i} C v_{j}$ or $v_{j} C^{-} v_{i}$. An $\ell$-circuit is a circuit with length $\ell$.

For a plane graph $G$ embedded in the plane $\Pi$, a face of $G$ is a connected topological region (an open set) of $\Pi \backslash G$. If the boundary of a face is a circuit of $G$, it is called a facial circuit of $G$. Denote $[1, k]=\{1,2, \ldots, k\}$. 


\subsubsection{Shrubberies}

Now we start to introduce shrubberies and removable circuits, which are key concepts for induction purpose.

Let $G$ be a signed graph and $H$ be a connected signed subgraph of $G$. An edge $e \in E(G) \backslash$ $E(H)$ is called a chord of $H$ if both ends of $e$ are in $V(H)$. We denote the set of chords of $H$ by $\mathcal{C}_{G}(H)$ or simply $\mathcal{C}(H)$, and partition $\mathcal{C}(H)$ into

$$
\mathcal{U}(H)=\mathcal{U}_{G}(H)=\{e \in \mathcal{C}(H): H+e \text { is unbalanced }\} \text { and } \overline{\mathcal{U}}(H)=\overline{\mathcal{U}_{G}}(H)=\mathcal{C}(H) \backslash \mathcal{U}(H) .
$$

A circuit $C$ is called removable if either it is unbalanced or it satisfies $|\mathcal{U}(C)|+\left|V_{2}(G) \cap V(C)\right| \geq 2$.

A signed graph $G$ is called a shrubbery if it satisfies the following requirements:

(S1) $\Delta(G) \leq 3$

(S2) every signed cubic subgraph of $G$ is flow-admissible;

(S3) $\left|\delta_{G}(V(H))\right|+\sum_{x \in V(H)}\left(3-d_{G}(x)\right)+2|\mathcal{U}(H)| \geq 4$ for any balanced and connected signed subgraph $H$ with $|V(H)| \geq 2$;

(S4) $G$ has no balanced 4-circuits.

The following proposition shows that shrubberies form a nice graph class which is closed under deletion, a crucial fact for induction.

Proposition 3.6.1. Every signed subgraph of a shrubbery is still a shrubbery.

Proof. Let $G^{\prime}$ be an arbitrary signed subgraph of a shrubbery $G$. Obviously, $G^{\prime}$ satisfies (S1), (S2) and (S4). We will show that $G^{\prime}$ satisfies (S3).

Let $H$ be a balanced and connected signed subgraph of $G^{\prime}$ with $|V(H)| \geq 2$. Let $A_{1}=$ $\delta_{G}(V(H)) \backslash \delta_{G^{\prime}}(V(H))$ and $A_{2}=\mathcal{C}_{G}(H) \backslash \mathcal{C}_{G^{\prime}}(H)$. Then

$$
\sum_{x \in V(H)}\left(3-d_{G^{\prime}}(x)\right)-\sum_{x \in V(H)}\left(3-d_{G}(x)\right)=\sum_{x \in V(H)}\left(d_{G}(x)-d_{H}(x)\right)=\left|A_{1}\right|+2\left|A_{2}\right| .
$$

Since $\mathcal{U}_{G^{\prime}}(H) \subseteq \mathcal{U}_{G}(H)$ and $\mathcal{C}_{G^{\prime}}(H) \subseteq \mathcal{C}_{G}(H)$, we have

$$
\left|\mathcal{U}_{G}(H)\right|-\left|\mathcal{U}_{G^{\prime}}(H)\right| \leq\left|A_{2}\right| .
$$

Hence

$$
\begin{aligned}
& \left|\delta_{G^{\prime}}(V(H))\right|+\sum_{x \in V(H)}\left(3-d_{G^{\prime}}(x)\right)+2\left|\mathcal{U}_{G^{\prime}}(H)\right| \\
\geq & \left(\left|\delta_{G}(V(H))\right|-\left|A_{1}\right|\right)+\left[\sum_{x \in V(H)}\left(3-d_{G}(x)\right)+\left|A_{1}\right|+2\left|A_{2}\right|\right]+2\left(\left|\mathcal{U}_{G}(H)\right|-\left|A_{2}\right|\right) \\
= & \left|\delta_{G}(V(H))\right|+\sum_{x \in V(H)}\left(3-d_{G}(x)\right)+2\left|\mathcal{U}_{G}(H)\right| \geq 4,
\end{aligned}
$$


since $G$ is a shrubbery.

Therefore $G^{\prime}$ satisfies (S3) and thus is a shrubbery.

Proposition 3.6.1 will be applied frequently in the proof of Lemma 3.6.10 and thus it will not be referenced explicitly.

Next we will apply the following two theorems and Lemma 3.6.4 to reduce Theorem 3.1.3 for general signed graphs to cubic shrubberies.

Theorem 3.6.1. ( [25]) Every ordinary bridgeless graph admits a 6-NZF.

Theorem 3.6.2. ( [28]) Let $A$ be an abelian group of order $k$. Then an ordinary graph admits a $k-N Z F$ if and only if it admits an $A-N Z F$.

Let $G$ be an ordinary oriented graph, $T \subseteq E(G)$ and $A$ be an abelian group. For any function $\gamma: T \rightarrow A$, let $\mathcal{F}_{\gamma}(G)$ denote the number of $A$-NZF $\phi$ of $\mathrm{G}$ with $\phi(e)=\gamma(e)$ for every $e \in T$. For every $X \subseteq V(G)$, let $\alpha_{X}: E(G) \rightarrow\{-1,0,1\}$ be given by the rule

$$
\alpha_{X}(e)=\left\{\begin{aligned}
1 & \text { if } e \in \delta_{G}(X) \text { is directed toward } X \\
-1 & \text { if } e \in \delta_{G}(X) \text { is directed away } X \\
0 & \text { otherwise. }
\end{aligned}\right.
$$

For any two functions $\gamma_{1}, \gamma_{2}$ from $T$ to $A$, we call $\gamma_{1}, \gamma_{2}$ similar if for every $X \subseteq V(G)$, the following holds

$$
\sum_{e \in T} \alpha_{X}(e) \gamma_{1}(e)=0 \text { if and only if } \sum_{e \in T} \alpha_{X}(e) \gamma_{2}(e)=0 .
$$

Lemma 3.6.3. (Seymour - Personal communication). Let $G$ be an ordinary oriented graph, $T \subseteq E(G)$ and $A$ be an abelian group. If the two functions $\gamma_{1}, \gamma_{2}: T \rightarrow A$ are similar, then $\mathcal{F}_{\gamma_{1}}(G)=\mathcal{F}_{\gamma_{2}}(G)$.

Proof. We proceed by induction on the number of edges in $E(G) \backslash T$. If this set is empty, then $\mathcal{F}_{\gamma_{i}}(G) \leq 1$ and $\mathcal{F}_{\gamma_{i}}(G)=1$ if and only if $\gamma_{i}$ is an $A$-NZF of $G$ for $i=1,2$. Thus, the result follows by the assumption. Otherwise, choose an edge $e \in E(G) \backslash T$. If $e$ is a cut-edge, then $\mathcal{F}_{\gamma_{i}}(G)=0$ for $i=1,2$. If $e$ is a loop, then we have inductively that

$$
\mathcal{F}_{\gamma_{1}}(G)=(|A|-1) \mathcal{F}_{\gamma_{1}}(G-e)=(|A|-1) \mathcal{F}_{\gamma_{2}}(G-e)=\mathcal{F}_{\gamma_{2}}(G)
$$

Otherwise, applying induction to $G-e$ and $G / e$ we have

$$
\mathcal{F}_{\gamma_{1}}(G)=\mathcal{F}_{\gamma_{1}}(G / e)-\mathcal{F}_{\gamma_{1}}(G-e)=\mathcal{F}_{\gamma_{2}}(G / e)-\mathcal{F}_{\gamma_{2}}(G-e)=\mathcal{F}_{\gamma_{2}}(G)
$$

The following lemma directly follows from Lemma 3.6.3. 
Lemma 3.6.4. Let $G$ be an ordinary oriented graph and $A$ be an abelian group. Assume that $G$ has an $A$-NZF. If $G$ has a vertex $v$ with $d_{G}(v) \leq 3$ and $\gamma: \delta_{G}(v) \rightarrow A \backslash\{0\}$ satisfies $\partial \gamma(v)=0$, then there exists an $A-N Z F \phi$ such that $\left.\phi\right|_{\delta_{G}(v)}=\gamma$.

Proof. Let $f$ be an $A$-NZF of $G$. Since $d_{G}(v) \leq 3,\left.f\right|_{\delta_{G}(v)}$ is similar to $\gamma$. Thus by Lemma 3.6.3, we have $\mathcal{F}_{\gamma}(G)=\mathcal{F}_{\left.f\right|_{\delta_{G}(v)}}(G) \neq 0$. Therefore there exists an $A$-NZF $\phi$ such that $\left.\phi\right|_{\delta_{G}(v)}=\gamma$.

Now we can reduce Theorem 3.1.3 to cubic shrubberies.

Lemma 3.6.5. The following two statements are equivalent.

(i) Every flow-admissible signed graph admits a balanced $\mathbb{Z}_{2} \times \mathbb{Z}_{3}-N Z F$.

(ii) Every cubic shrubbery admits a balanced $\mathbb{Z}_{2} \times \mathbb{Z}_{3}-N Z F$.

Proof. "(i) $\Rightarrow($ ii)": By (S2), every cubic shrubbery is flow-admissible, and thus (ii) follows from (i).

"(ii) $\Rightarrow$ (i)": Let $G$ be a counterexample to (i) with $\beta(G)=\sum_{v \in V(G)}\left|d_{G}(v)-2.5\right|$ minimum. Since $G$ is flow-admissible, it admits a $k$-NZF $(\tau, f)$ for some positive integer $k$ and thus $V_{1}(G)=$ $\emptyset$. Furthermore, by the minimality of $\beta(G), G$ is connected and $V_{2}(G)=\emptyset$ otherwise the suppressed signed graph $\bar{G}$ of $G$ is also flow-admissible and has smaller $\beta(\bar{G})$ than $\beta(G)$. We are going to show that $G$ is a cubic shrubbery and thus admits a balanced $\mathbb{Z}_{2} \times \mathbb{Z}_{3}$-NZF by (ii), which is a contradiction to the fact that $G$ is a counterexample. By the definition of shrubberies, we only need to prove (I)-(III) in the following.

(I) $G$ is cubic.

Suppose to the contrary that $G$ has a vertex $v$ with $d_{G}(v) \neq 3$. Then $d_{G}(v) \geq 4$. Let $\left\{e_{1}, e_{2}\right\} \subset \delta_{G}(v)$ and let $G^{\prime}=G_{\left[v ;\left\{e_{1}, e_{2}\right\}\right]}$. Denote the new common end of $e_{1}$ and $e_{2}$ in $G^{\prime}$ by $v^{*}$. If $\partial f\left(v^{*}\right)=0$, let $G^{\prime \prime}=G^{\prime}$. If $\partial f\left(v^{*}\right) \neq 0$, we further add a positive edge $v v^{*}$ with direction from $v$ to $v^{*}$ and assign $v v^{*}$ with flow value $\partial f\left(v^{*}\right)$. Let $G^{\prime \prime}$ be the resulting signed graph. In both cases, $G^{\prime \prime}$ is flow-admissible and $\beta\left(G^{\prime \prime}\right)<\beta(G)$. By the minimality of $\beta(G), G^{\prime \prime}$ admits a balanced $\mathbb{Z}_{2} \times \mathbb{Z}_{3}-\mathrm{NZF}$, and so does $G$, a contradiction. This proves (I).

(II) $\left|\delta_{G}(V(H))\right|+2|\mathcal{U}(H)| \geq 4$ for any balanced and connected signed subgraph $H$ with $|V(H)| \geq$ 2 .

Suppose to the contrary that $H$ is such a subgraph with $\left|\delta_{G}(V(H))\right|+2|\mathcal{U}(H)| \leq 3$. Let $X=V(H)$. Then $H^{\prime}=G[X]-\mathcal{U}(H)$ is a balanced and connected signed subgraph of $G$. WLOG assume that all edges of $H^{\prime}$ are positive. Let $G_{1}=G / E\left(H^{\prime}\right)$. Then $G_{1}$ is also flow-admissible.

Since $\left|\delta_{G}(X)\right|+2|\mathcal{U}(H)| \leq 3$, it follows from the choice of $G$ and Proposition 3.2.1 that either $|\mathcal{U}(H)|=0$ and $\left|\delta_{G}(X)\right| \in\{2,3\}$ or $|\mathcal{U}(H)|=1$ and $\left|\delta_{G}(X)\right|=1$. Let $x$ be the contracted vertex in $G_{1}=G / E\left(H^{\prime}\right)$ corresponding to $E\left(H^{\prime}\right)$. Then $d_{G_{1}}(x)=\left|\delta_{G}(X)\right|+2|\mathcal{U}(H)| \in\{2,3\}$ 
and $\beta\left(G_{1}\right)<\beta(G)$ since $|X|=|V(H)| \geq 2$. By the minimality of $\beta(G), G_{1}$ admits a balanced $\mathbb{Z}_{2} \times \mathbb{Z}_{3}$-NZF $\left(\tau_{1}, f_{1}\right)$, where $\tau_{1}$ is the restriction of $\tau$ on $G_{1}$.

Let $H_{X}$ be the set of the half edges of each edge in $\delta_{G}(X) \cup \mathcal{U}(H)$ whose end is in $X$. Then $\left|H_{X}\right|=\left|\delta_{G}(X)\right|+2|\mathcal{U}(H)|=2$ or 3 . Construct a new graph $G_{2}$ from $H^{\prime}+H_{X}$ by identifying the non-ends of all half edges in $H_{X}$ into a new vertex $y$. Now in $G_{2}, y$ is the common end of all $h \in H_{x}$. Then in $G_{2}, y$ is the vertex incident with all $h \in H_{X}$. Since $G$ is flow-admissible, $G_{2}$ is a bridgeless ordinary graph and thus admits a balanced $\mathbb{Z}_{2} \times \mathbb{Z}_{3}$-NZF by Theorems 3.6.1 and 3.6.2. Let $\tau_{2}$ be the restriction of $\tau$ on $G_{2}$ and define $\gamma(h)=f_{1}\left(e_{h}\right)$ for each $h \in H_{X}$. Note that $\tau_{2}(h)=\tau_{1}(h)$ for each $h \in H_{X}$. Since $\left(\tau_{1}, f_{1}\right)$ is a balanced $\mathbb{Z}_{2} \times \mathbb{Z}_{3}$-NZF of $G_{1}$, we have $\partial \gamma(y)=-\partial f_{1}(x)=0$. By Lemma 3.6.4, there is a balanced $\mathbb{Z}_{2} \times \mathbb{Z}_{3}$-NZF $\left(\tau_{2}, f_{2}\right)$ of $G_{2}$ such that $\left.f_{2}\right|_{\delta_{G_{2}}(y)}=\gamma=\left.f_{1}\right|_{\delta_{G_{1}}(x)}$. Thus $\left(\tau_{1}, f_{1}\right)$ can be extended to a balanced $\mathbb{Z}_{2} \times \mathbb{Z}_{3}$-NZF of $G$, a contradiction. This proves (II).

(III) $G$ has no balanced 4-circuits.

Suppose to the contrary that $G$ has a balanced 4-circuit $C$. Then we may assume that all edges of $C$ are positive. Let $G^{\prime}=G / E(C)$. Then $\beta\left(G^{\prime}\right)<\beta(G)$. By the minimality of $\beta(G)$, $G^{\prime}$ admits a balanced $\mathbb{Z}_{2} \times \mathbb{Z}_{3}$-NZF, say $\left(f_{1}^{\prime}, f_{2}^{\prime}\right)$. Since $C$ is a circuit with all positive edges and $|E(C)|=4$ and since $\left|\mathbb{Z}_{2} \times \mathbb{Z}_{3}\right|=6$, it is easy to extend $\left(f_{1}^{\prime}, f_{2}^{\prime}\right)$ to a balanced $\mathbb{Z}_{2} \times \mathbb{Z}_{3}$-NZF of $G$, a contradiction. This proves (III) and thus completes the proof of the lemma.

\subsubsection{Nowhere-zero watering}

In this subsection, we will prove that every cubic shrubbery admits a balanced $\mathbb{Z}_{2} \times \mathbb{Z}_{3}$-NZF. In fact, we will prove a stronger result that every shrubbery admits a nowhere-zero watering as in Lemma 3.6.10 below. Here a nowhere-zero watering (see Definition 3.6.1) involves flows with certain boundaries at vertices of degree one or two, which provides some flexibility for induction and makes some reduction arguments on removable circuits possible. Before proceeding, we need some preparations.

Theorem 3.6.6. ( [31]) Let $G$ be a 2-connected graph with $\Delta(G) \leq 3$ and let $y_{1}, y_{2}, y_{3} \in V(G)$. Then either there exists a circuit of $G$ containing $y_{1}, y_{2}, y_{3}$, or there is a partition of $V(G)$ into $\left\{X_{1}, X_{2}, Y_{1}, Y_{2}, Y_{3}\right\}$ with the following properties:

(1) $y_{i} \in Y_{i}$ for $i=1,2,3$;

(2) $\delta_{G}\left(X_{1}, X_{2}\right)=\delta_{G}\left(Y_{i}, Y_{j}\right)=\emptyset$ for $1 \leq i<j \leq 3$;

(3) $\left|\delta_{G}\left(X_{i}, Y_{j}\right)\right|=1$ for $i=1,2$ and $j=1,2,3$.

Let $H$ be a contraction of $G$ and let $x \in V(G)$. We use $\hat{x}$ to denote the vertex in $H$ which $x$ is contracted into. 
Theorem 3.6.7. ( $[21])$ Let $G$ be a 2-connected signed graph with $\left|E_{N}(G)\right|=\epsilon(G)=k \geq 2$, where $E_{N}(G)=\left\{x_{1} x_{k+1}, \ldots, x_{k} x_{2 k}\right\}$. Then the following two statements are equivalent.

(i) $G$ does not contain two edge-disjoint unbalanced circuits.

(ii) The graph $G$ can be contracted to a cubic graph $G^{\prime}$ such that either $G^{\prime}-\left\{\hat{x}_{1} \hat{x}_{k+1}, \ldots, \hat{x}_{k} \hat{x}_{2 k}\right\}$ is a $2 k$-circuit $C_{1}$ on the vertices $\hat{x}_{1}, \ldots, \hat{x}_{k}, \hat{x}_{k+1}, \ldots, \hat{x}_{2 k}$ or can be obtained from a 2 connected cubic plane graph by selecting a facial circuit $C_{2}$ and inserting the vertices $\hat{x}_{1}, \ldots, \hat{x}_{k}, \hat{x}_{k+1}, \ldots, \hat{x}_{2 k}$ on the edges of $C_{2}$ in such a way that for every pair $\{i, j\} \subseteq[1, k]$, the vertices $\hat{x}_{i}, \hat{x_{j}}, x_{\hat{k+i}}, x_{\hat{k+j}}$ are around the circuit $C_{1}$ or $C_{2}$ in this cyclic order.

Lemma 3.6.8. ( [19]) Let $G$ be an ordinary oriented graph and $A$ be an abelian group. Then $G$ is connected if and only if for every function $\beta: V(G) \rightarrow A$ satisfying $\sum_{v \in V(G)} \beta(v)=0$, there exists $\phi: E(G) \rightarrow A$ such that $\partial \phi=\beta$.

Definition 3.6.1. Let $G$ be a signed graph with $\Delta(G) \leq 3$ and a given orientation. A nowherezero watering (briefly, $N Z W$ ) of $G$ is a mapping $f: E(G) \rightarrow \mathbb{Z}_{2} \times \mathbb{Z}_{3}-\{(0,0)\}$ such that

$$
\partial f(v)=(0,0) \text { if } d_{G}(v)=3 \text { and } \partial f(v)=(0, \pm 1) \text { if } d_{G}(v)=1,2 .
$$

Similar to flows, the existence of an NZW is also an invariant under switching operation. The following reductions/extensions of NZW on removable circuits play an important role in later proofs.

Lemma 3.6.9. Let $G$ be a shrubbery and $C$ be a removable circuit of $G$. Then for every $N Z W$ $f^{\prime}=\left(f_{1}^{\prime}, f_{2}^{\prime}\right)$ of $G^{\prime}=G-V(C)$, there exists an $N Z W f=\left(f_{1}, f_{2}\right)$ of $G$ so that $f(e)=f^{\prime}(e)$ for every $e \in E\left(G^{\prime}\right)$ and $\operatorname{supp}\left(f_{1}\right)=\operatorname{supp}\left(f_{1}^{\prime}\right) \cup E(C)$.

Proof. We first extend $f^{\prime}$ to $f: E(G) \rightarrow \mathbb{Z}_{2} \times \mathbb{Z}_{3}$ as follows where $\alpha_{e}$ is a variable in $\mathbb{Z}_{3}$ for every $e \in \mathcal{U}(C)$.

$$
f(e)= \begin{cases}(0, \pm 1) & \text { if } e \in \delta(V(C)) \\ (1,0) & \text { if } e \in E(C) \\ (0,1) & \text { if } e \in \overline{\mathcal{U}}(C) \\ \left(0, \alpha_{e}\right) & \text { if } e \in \mathcal{U}(C) .\end{cases}
$$

Since every $v \in V(G) \backslash V(C)$ adjacent to a vertex in $V(C)$ has degree less than three in $G^{\prime}$, we may choose values $f(e)$ for each edge $e \in \delta(V(C))$ so that $f$ satisfies the boundary condition for a watering at every vertex in $V(G) \backslash V(C)$. Obviously by the construction $\partial f_{1}(v)=0$ for every $v \in V(C)$. So we need only adjust $\partial f_{2}(v)$ for $v \in V(C)$ to obtain a watering. We distinguish the following two cases.

Case 1: $C$ is unbalanced. 
In this case $\overline{\mathcal{U}}(C)=\emptyset$. Choose arbitrary \pm 1 assignments to the variables $\alpha_{e}$. Since $C$ is unbalanced, for every vertex $u \in V(C)$, there is a function $\eta_{u}: E(C) \rightarrow \mathbb{Z}_{3}$ so that $\partial \eta_{u}(u)=1$ and $\partial \eta_{u}(v)=0$ for any $v \in V(C) \backslash\{u\}$. Now we may adjust $f_{2}$ by adding a suitable combination of the $\eta_{u}$ functions so that $f$ is an NZW of $G$, as desired.

Case 2: $C$ is balanced.

WLOG we may assume that every edge of $C$ is positive and every unbalanced chord is oriented so that each half edge is directed away from its end. In this case, each negative chord $e$ contributes $2 f_{2}(e)=\alpha_{e}$ to the sum $\sum_{v \in V(C)} \partial f_{2}(v)$. For every $v \in V(C) \cap V_{2}(G)$, let $\beta_{v}$ be a variable in $\mathbb{Z}_{3}$. Since $|\mathcal{U}(C)|+\left|V_{2}(G) \cap V(C)\right| \geq 2$, we can choose \pm 1 assignments to all of the variables $\alpha_{e}$ and $\beta_{v}$ so that the following equation is satisfied:

$$
\sum_{v \in V(C)} \partial f_{2}(v)=\sum_{v \in V(C) \cap V_{2}(G)} \beta_{v} .
$$

By Lemma 3.6.8, we may choose a function $\phi: E(C) \rightarrow \mathbb{Z}_{3}$ so that

$$
\partial \phi(v)=\left\{\begin{array}{cc}
\beta_{v}-\partial f_{2}(v) & \text { if } v \in V(C) \cap V_{2}(G), \\
-\partial f_{2}(v) & \text { if } v \in V(C) \backslash V_{2}(G) .
\end{array}\right.
$$

Now modify $f$ by adding $\phi$ to $f_{2}$ and then $f$ is an NZW of $G$, as desired.

A theta is a graph consisting of two distinct vertices and three internally disjoint paths between them. A theta is unbalanced if it contains an unbalanced circuit. By the definition, the following observation is straightforward.

Observation 3.6.1. Let $G$ be a signed graph containing no unbalanced thetas and $\Delta(G) \leq 3$. Then for any unbalanced circuit $C$ and any $x \in V(G) \backslash V(C), G$ does not contain two internally disjoint $(x, C)$-paths.

Now we present our main result of this subsection.

Lemma 3.6.10. Every shrubbery has an NZW. Furthermore, if $G$ is a shrubbery with an unbalanced theta or a negative loop and $\varepsilon \in\{-1,1\}$, then $G$ has an $N Z W f=\left(f_{1}, f_{2}\right)$ such that $\sigma\left(\operatorname{supp}\left(f_{1}\right)\right)=\varepsilon$.

Before we go through the details of the proof, we first present the outline of the proof.

Outline of the proof of Lemma 3.6.10: Consider $G$ the minimum counterexample to the lemma. If $G$ does not contain an unbalanced theta or a negative loop, all removable circuits are forbidden from $G$ (See Claim 3.6.2-(1)). However due to the requirement of $\epsilon$, if $G$ has an unbalanced theta or a negative loop, only removable circuits with certain properties can be forbidden from $G$ (See Claim 3.6.2-(2a) and (2b)). 
Thus, in order to avoid "forbidden circuits", certain structures of $G$ are determined stepby-step in Claims 3.6.3-3.6.8, especially, the non-existence of edge-disjoint unbalanced circuits (Claim 3.6.6). With those structures and the application of Theorem 3.6.7, we are able to lead the final contradiction that some forbidden circuit does exist in the remaining part of the proof (Claims 3.6.9-3.6.11 and the final step).

Proof. Let $G$ be a minimum counterexample with respect to $|E(G)|$. Then $G$ is connected.

Claim 3.6.1. $\Delta(G)=3$ and $G$ is 2-connected. Thus $G$ does not contain loops.

Proof of Claim 3.6.1. It is obvious that both a circuit (balanced or unbalanced) and a path have NZWs. Since $\Delta(G) \leq 3$ by (S1), we have $\Delta(G)=3$.

Now we show that $G$ is 2 -connected. Suppose to the contrary that $G$ has a cut vertex. Since $\Delta(G)=3, G$ contains a cut-edge $e=v_{1} v_{2}$. Let $G_{i}$ be the component of $G-e$ containing $v_{i}$. By the minimality of $G$, each $G_{i}$ admits an NZW $f^{i}=\left(f_{1}^{i}, f_{2}^{i}\right)$, and $\partial f_{2}^{i}\left(v_{i}\right) \neq 0$ since $d_{G_{i}}\left(v_{i}\right) \leq 2$. Thus we can obtain an NZW $f=\left(f_{1}, f_{2}\right)$ of $G$ by setting $f(e)=(0,1)$ and $\left.f\right|_{E\left(G_{i}\right)}=f^{i}$ or $-f^{i}$ according to the orientation of $e$ and the values of $\partial f_{2}^{1}\left(v_{1}\right)$ and $\partial f_{2}^{2}\left(v_{2}\right)$. Further, if $G$ contains an unbalanced theta or a negative loop, so does one component of $G-e$, say $G_{1}$. By the minimality of $G$, we choose $f^{1}$ such that $\sigma\left(\operatorname{supp}\left(f_{1}^{1}\right)\right)=\epsilon \cdot \sigma\left(\operatorname{supp}\left(f_{1}^{2}\right)\right)$. Hence $\sigma\left(\operatorname{supp}\left(f_{1}\right)\right)=\sigma\left(\operatorname{supp}\left(f_{1}^{1}\right)\right) \cdot \sigma\left(\operatorname{supp}\left(f_{1}^{2}\right)\right)=\epsilon \cdot \sigma\left(\operatorname{supp}\left(f_{1}^{2}\right)\right) \cdot \sigma\left(\operatorname{supp}\left(f_{1}^{2}\right)\right)=\epsilon$, a contradiction.

Claim 3.6.2. (1) If $G$ does not contain an unbalanced theta, then $G$ doesn't not contain a removable circuit.

(2) If $G$ contains an unbalanced theta, then $G$ has no removable circuit $C$ with one of the following properties:

(2a) $G-V(C)$ contains an unbalanced theta;

(2b) $G-V(C)$ is balanced and $\sigma(C)=\epsilon$.

Proof of Claim 3.6.2. Note that $G$ does not contain a negative loop.

(1) is straightforward from Lemma 3.6.9

Suppose that (2) is not true. Then $G$ contains an unbalanced theta. Let $C$ be a removable circuit satisfying (2a) or (2b). By the minimality of $G$, there exists an NZW $f^{\prime}=\left(f_{1}^{\prime}, f_{2}^{\prime}\right)$ of $G-V(C)$ such that $\sigma\left(\operatorname{supp}\left(f_{1}^{\prime}\right)\right)=\epsilon \cdot \sigma(C)$ in Case $(2 \mathrm{a})$ and $\sigma\left(\operatorname{supp}\left(f_{1}^{\prime}\right)\right)=1$ in Case (2b). By Lemma 3.6.9, $f^{\prime}$ can be extended to an NZW $f=\left(f_{1}, f_{2}\right)$ of $G$ such that $\operatorname{supp}\left(f_{1}\right)=$ $\operatorname{supp}\left(f_{1}^{\prime}\right) \cup E(C)$. In particular for Cases (2a) and $(2 \mathrm{~b}), \sigma\left(\operatorname{supp}\left(f_{1}\right)\right)=\sigma\left(\operatorname{supp}\left(f_{1}^{\prime}\right)\right) \cdot \sigma(C)=\epsilon$, a contradiction.

Claim 3.6.3. Let $X \subset V(G)$ such that $|X| \geq 2, G[X]$ is balanced, and $\left|\delta_{G}(X)\right|=2$. If $G-X$ either contains an unbalanced theta, or is balanced and contains a circuit, then $X \subseteq V_{2}(G)$ and thus $G[X]$ is a path. 
Proof of Claim 3.6.3. The conclusion that $G[X]$ is a path directly follows from the properties of $X$ and the first conclusion that $X \subseteq V_{2}(G)$.

Suppose the claim fails. Let $X \subset V(G)$ be a minimal set with the above properties such that $X \cap V_{3}(G) \neq \emptyset$. Then $G[X]$ is 2 -connected by the minimality of $X$. Since $G[X]$ is balanced and $\mathcal{U}(G[X])=\emptyset$, by (S3), we have

$$
2+\sum_{x \in X}\left(3-d_{G}(x)\right)=\left|\delta_{G}(X)\right|+\sum_{x \in X}\left(3-d_{G}(x)\right)+2|\mathcal{U}(G[X])| \geq 4 .
$$

The above inequality implies that $X$ contains at least two 2-vertices. Since $G[X]$ is 2-connected, let $C$ be a circuit in $G[X]$ containing at least two 2-vertices. Then $C$ is removable and thus by Claim 3.6.2-(2a), $G-V(C)$ does not contain a unbalanced theta, which implies that $G-X$ does not contain unbalanced theta either. By the hypothesis, $G-X$ is balanced and $G-X$ contains a circuit too.

Denote $\delta_{G}(X)=\left\{e_{1}, e_{2}\right\}$. Since both $G[X]$ and $G-X$ are balanced, by possibly replacing $\sigma_{G}$ with an equivalent signature, we may assume that $\sigma_{G}\left(e_{1}\right) \in\{-1,1\}$ and that $\sigma_{G}(e)=1$ for every other edge $e \in E(G)$. Since $C$ is a removable circuit of $G, G$ contains an unbalanced theta by Claim 3.6.2-(1), and so $G$ is unbalanced. Therefore $\sigma_{G}\left(e_{1}\right)=-1$ and thus $e_{1}$ is the only negative edge in $G$.

Let $C^{\prime}$ be an unbalanced circuit and $C^{\prime \prime}$ be a circuit in $G-X$. Then $C^{\prime \prime}$ is balanced and $C^{\prime}$ contains $e_{1}$ and $e_{2}$.

Now we show that $C^{\prime} \cup(G-X)$ contains an unbalanced theta. Denote $e_{1}=x_{1} y_{1}$ and $e_{2}=x_{2} y_{2}$, where $x_{1}, x_{2} \in X$ and $y_{1}, y_{2} \in V(G) \backslash X$. Since $G$ is 2-connected and $\Delta(G)=3$, there are two disjoint $\left(x_{1}, C^{\prime \prime}\right)$-paths $P_{1}$ and $P_{2}$ with $V\left(P_{1}\right) \cap V\left(P_{2}\right)=\left\{x_{1}\right\}$. Since $C^{\prime}$ contains both $e_{1}$ and $e_{2}$, we may choose $P_{1}$ and $P_{2}$ such that $P_{1} \cup P_{2}$ contains the segment of $C^{\prime}$ in $G[X]$ from $x_{1}$ to $x_{2}$. Since $e_{1}$ is the only negative edge, $P_{1} \cup P_{2} \cup C^{\prime \prime}$ is an unbalanced theta.

Since $C^{\prime}$ is unbalanced, it is removable. Since $G-V\left(C^{\prime}\right)$ is balanced and $\sigma\left(C^{\prime}\right)=-1$, by Claim 3.6.2-(2b), we have $\epsilon=1$. On the other hand, since $C$ is removable and $\sigma_{G}(C)=1=\epsilon$, $G-V(C)$ is unbalanced by Claim 3.6.2-(2b) again. Thus we may choose the unbalanced circuit $C^{\prime}$ in $G-V(C)$. Hence $V\left(C^{\prime}\right) \cap V(C)=\emptyset$. Therefore $P_{1} \cup P_{2} \cup C^{\prime \prime}$ is an unbalanced theta in $G-V(C)$, a contradiction to Claim 3.6.2-(2a).

Claim 3.6.4. Let $X \subset V(G)$ such that $|X| \geq 2, G[X]$ is balanced, and $\left|\delta_{G}(X)\right| \leq 3$. For any two distinct ends $x_{1}, x_{2}$ in $X$ of $\delta_{G}(X)$, there is an $\left(x_{1}, x_{2}\right)$-path in $G[X]$ containing at least one vertex in $V_{2}(G)$.

Proof of Claim 3.6.4. Suppose that the claim fails. Let $x_{1} x_{1}^{\prime}, x_{2} x_{2}^{\prime} \in \delta_{G}(X)$, and $B_{i}$ be the maximal 2-connected subgraph of $G[X]$ containing $x_{i}$ for $i=1,2$. Since $G$ is 2-connected and $\Delta(G)=3$ by Claim 3.6.1 and $\left|\delta_{G}(X)\right| \leq 3$, we have that $G[X]$ is connected and $d_{G}\left(x_{1}\right)=$ $d_{G}\left(x_{2}\right)=3$. Moreover every edge in $\delta_{G[X]}\left(V\left(B_{i}\right)\right)$ is a cut-edge of $G[X]$ by the maximality of 
$B_{i}$. Thus $\left|\delta_{G[X]}\left(V\left(B_{i}\right)\right)\right|$ is equal to the number of components of $G[X]-V\left(B_{i}\right)$. Since $G$ is 2-connected, we have

(a) for each component $A$ of $G[X]-V\left(B_{i}\right), \delta_{G}(V(A), V(G) \backslash X) \geq 1$ and thus

(b) $\left|\delta_{G}\left(V\left(B_{i}\right)\right)\right| \leq\left|\delta_{G}(X)\right| \leq 3$.

Moreover, since $G[X]$ is balanced, $B_{i}$ is balanced for $i=1,2$. Thus we further have

(c) $\mathcal{U}\left(B_{i}\right)=\emptyset$ for $i=1,2$.

We first show that for each $i=1,2 B_{i}$ does not contain a 2 -vertex and is trivial .

WLOG, suppose to the contrary that $B_{1}$ contains a 2-vertex $y$.

If $x_{2} \in V\left(B_{1}\right)$, then there are two internally disjoint $\left(y,\left\{x_{1}, x_{2}\right\}\right)$-paths $P_{1}$ and $P_{2}$. Then $P_{1} \cup P_{2}$ is an $\left(x_{1}, x_{2}\right)$-path in $G[X]$ containing one 2-vertex.

If $x_{2} \notin V\left(B_{1}\right)$, then $B_{1}$ and $B_{2}$ are disjoint since $\Delta(G)=3$. Since $G[X]$ is connected, let $P_{3}$ be an $\left(x_{2}, B_{1}\right)$-path and $y_{1}$ be the other end of $P_{3}$. Then $y_{1} \in V\left(B_{1}\right)$. Again since $B_{1}$ is 2-connected and $d_{G}\left(x_{1}\right)=3, y_{1} \neq x_{1}$ and there are two internally disjoint $\left(y,\left\{y_{1}, x_{1}\right\}\right)$-paths, $P_{1}^{\prime}$ and $P_{2}^{\prime}$. Then $P_{3} \cup P_{1}^{\prime} \cup P_{2}^{\prime}$ is a desired $\left(x_{1}, x_{2}\right)$-path. This proves that $B_{1}$ (and $\left.B_{2}\right)$ doesn't contain a 2 -vertex.

By (b) and (c), we have $\left|\delta_{G}\left(V\left(B_{i}\right)\right)\right| \leq 3$ and $\mathcal{U}\left(B_{i}\right)=\emptyset$ for $i=1,2$. If $B_{i}$ is nontrivial, then by (S3), we have

$$
4 \leq \sum_{x \in V\left(B_{i}\right)}\left(3-d_{G}(x)\right)+\left|\delta_{G}\left(V\left(B_{1}\right)\right)\right| \leq \sum_{x \in V\left(B_{i}\right)}\left(3-d_{G}(x)\right)+3 .
$$

The above inequality implies that $B_{i}$ contains a 2-vertex, a contradiction. Therefore $B_{i}$ is trivial.

Since $d_{G}\left(x_{1}\right)=3, d_{G[X]}\left(x_{1}\right)=2$ and thus $G[X]-x_{1}$ has two components, say $A_{1}$ and $A_{2}$. WLOG, we may assume $x_{2} \in V\left(A_{2}\right)$. Since $G$ is 2-connected, there exists $x_{3} x_{3}^{\prime} \in$ $\delta_{G}\left(V\left(A_{1}\right), V(G) \backslash X\right)$ with $x_{3} \in V\left(A_{1}\right)$. Similarly, $G[X]-x_{2}$ has two components $A_{3}$ and $A_{4}$. Since $G[X]$ is connected, the subgraph induced by $V\left(A_{1}\right)$ together with $x_{1}$ must be contained in one of $A_{3}$ and $A_{4}$, say $A_{4}$. Thus $\delta_{G}\left(V\left(A_{4}\right), V(G) \backslash X\right)=\left\{x_{1} x_{1}^{\prime}, x_{3} x_{3}^{\prime}\right\}$. Note that $\delta_{G}(X)=\left\{x_{1} x_{1}^{\prime}, x_{2} x_{2}^{\prime}, x_{3} x_{3}^{\prime}\right\}$ since $\left|\delta_{G}(X)\right| \leq 3$. Since $x_{2} \notin V\left(A_{3}\right), \delta\left(V\left(A_{3}\right), V(G) \backslash X\right)=0<1$, a contradiction to (a). This proves the claim.

Claim 3.6.5. $G$ does not contain two disjoint unbalanced circuits $C_{1}$ and $C_{2}$ such that $V_{3}(G) \subseteq$ $V\left(C_{1}\right) \cup V\left(C_{2}\right)$.

Proof of Claim 3.6.5. Suppose the claim fails. Let $C_{1}$ and $C_{2}$ be two disjoint unbalanced circuits such that $V_{3}(G) \subseteq V\left(C_{1}\right) \cup V\left(C_{2}\right)$. Then every vertex of $G^{\prime}=G-E\left(C_{1} \cup C_{2}\right)$ is of degree at most 2. By Claim 3.6.2-(2a), $G-V\left(C_{i}\right)$ does not contain unbalanced theta for each $i=1,2$. Thus by Observation 3.6.1, every nontrivial component of $G^{\prime}$ is a path with one end in $V\left(C_{1}\right)$ and the other end in $V\left(C_{2}\right)$. Since $G$ is 2-connected and $\Delta(G)=3$, there are at least two 3 -vertices in each $C_{i}$. 
When $\epsilon=-1$, choose $x_{1}, x_{2}$ from $V_{3}(G) \cap V\left(C_{1}\right)$ such that the segment $P=x_{1} C_{1} x_{2}$ contains all vertices of $V_{3}(G) \cap V\left(C_{1}\right)$. Let $P_{i}$ be the path in $G^{\prime}$ with one end $x_{i}$ and $y_{i}$ be the other end of $P_{i}$ for $i=1,2$. Since $C_{2}$ is unbalanced, there is a segment, say $y_{1} C_{2} y_{2}$, of $C_{2}$ such that the circuit $C=P \cup P_{1} \cup P_{2} \cup y_{1} C_{2} y_{2}$ is unbalanced, and thus $C$ is removable. This contradicts Claim 3.6.2-(2b) since $G-V(C)$ is a forest (which is balanced).

When $\epsilon=1$, by the minimality of $G$ and since $G^{\prime \prime}=G-V\left(C_{1} \cup C_{2}\right)$ is a forest, $G^{\prime \prime}$ admits an NZW $f^{\prime}=\left(f_{1}^{\prime}, f_{2}^{\prime}\right)$ with $\operatorname{supp}\left(f_{1}^{\prime}\right)=\emptyset$. By applying Lemma 3.6.9 twice, we extend $f^{\prime}=\left(f_{1}^{\prime}, f_{2}^{\prime}\right)$ to an NZW $f=\left(f_{1}, f_{2}\right)$ of $G$ such that $\operatorname{supp}\left(f_{1}\right)=E\left(C_{1}\right) \cup E\left(C_{2}\right)$. So $\sigma\left(\operatorname{supp}\left(f_{1}\right)\right)=$ $\sigma\left(C_{1}\right) \cdot \sigma\left(C_{2}\right)=1=\epsilon$, a contradiction.

Claim 3.6.6. $G$ does not contain two disjoint unbalanced circuits.

Proof of Claim 3.6.6. Suppose to the contrary that $C_{1}$ and $C_{2}$ are two disjoint unbalanced circuits of $G$. By Claim 3.6.5, $V_{3}(G) \backslash V\left(C_{1} \cup C_{2}\right) \neq \emptyset$.

Let $x \in V_{3}(G) \backslash V\left(C_{1} \cup C_{2}\right)$. By Claim 3.6.2-(2a), for each $C_{i}, G-V\left(C_{i}\right)$ does not contain an unbalanced theta. Thus by Observation 3.6.1, there exists a 2-edge-cut of $G$ separating $x$ from $V\left(C_{1} \cup C_{2}\right)$. Let $\left\{e_{1}, e_{2}\right\}$ be such a 2-edge-cut. Let

$$
\mathcal{F}=\left\{e_{1}\right\} \cup\left\{e \in E(G):\left\{e, e_{1}\right\} \text { is a 2-edge-cut of } G\right\}
$$

and $\mathcal{B}$ be the set of all nontrivial components of $G-\mathcal{F}$. Then every member of $\mathcal{B}$ is 2-connected. Since $d_{G}(x)=3$, there is a $B_{0} \in \mathcal{B}$ containing $x$.

We claim that $\mathcal{B}$ has the following properties:

(a) Each $B \in \mathcal{B}$ contains a removable circuit. In particular, if $B$ is balanced, then $B$ contains at least one 2-vertex.

(b) Each $B \in \mathcal{B}$ is either balanced or is an unbalanced circuit.

(c) $|\mathcal{B}| \geq 3$.

Let $B \in \mathcal{B}$. Then $\left|\delta_{G}(V(B))\right|=2$ and $\mathcal{U}(B)=\emptyset$. If $B$ is balanced, then by (S3), $B$ contains at least two 2-vertices and thus contains a circuit containing at least two 2-vertices which is removable. If $B$ is unbalanced, then $B$ contains an unbalanced circuit which is also removable. This proves (a).

Since $B_{0}$ doesn't contain $C_{1}$ or $C_{2},|\mathcal{B}| \geq 2$. By (a) each member $B$ in $\mathcal{B}$ contains a removable circuit. Thus by Claim 3.6.2-(2a), each member of $\mathcal{B}$ does not contain unbalanced theta and so is an unbalanced circuit if it is unbalanced. This proves (b)

By (b), $C_{1}$ and $C_{2}$ belong to distinct members in $\mathcal{B}$. Note that $B_{0}$ doesn't contain $C_{1}$ or $C_{2}$. Thus $|\mathcal{B}| \geq 3$. This proves (c).

Since $G$ is 2-connected, there is a circuit that contains all edges in $\mathcal{F}$ and goes through every $B \in \mathcal{B}$. We choose such a circuit $C$ with the following properties:

(1) $\sigma(C)=\epsilon$ (the existence of $C$ is guaranteed since $C_{1}$ is unbalanced); 
(2) subject to (1), $\left|V_{2}(G) \cap V\left(C-V\left(C_{1}\right)\right)\right|$ is as large as possible;

(3) subject to (1) and (2), $\left|E_{N}(G) \cap E\left(C-V\left(C_{1}\right)\right)\right|$ is as small as possible.

We claim that $C$ is removable.

Let $B \in \mathcal{B} \backslash\left\{C_{1}\right\}$. If $B$ is balanced, then by (a), $B$ contains a 2 -vertex. Since $B$ is 2connected, by (2), $C$ contains at least one 2 -vertex in $B$. If $B$ is an unbalanced circuit of length at least 3 , then by (2), $C$ contains one 2 -vertex in $B$ too. If $B$ is an unbalanced circuit of length 2, then by (3), $C$ contains the positive edge in $B$ and the negative edge in $B$ belongs to $\mathcal{U}(C)$. Therefore every $B \in \mathcal{B} \backslash\left\{C_{1}\right\}$ contributes at least 1 to $|\mathcal{U}(C)|+\left|V_{2}(G) \cap V(C)\right|$. Since $\left|\mathcal{B} \backslash\left\{C_{1}\right\}\right| \geq 2$, we have $|\mathcal{U}(C)|+\left|V_{2}(G) \cap V(C)\right| \geq 2$. Hence $C$ is a removable circuit.

Since each $B \in \mathcal{B}$ is either balanced or an unbalanced circuit, $G-V(C)$ is balanced. This contradicts Claim 3.6.2-(2b) since $C$ is removable and since $\sigma(C)=\epsilon$ by (1).

Claim 3.6.7. $G$ contains an unbalanced theta and $\epsilon=1$.

Proof of Claim 3.6.7. We first show that $G$ contains an unbalanced theta.

Suppose that $G$ does not contain unbalanced theta. If $G$ is unbalanced, then it contains an unbalanced circuit. If $G$ is balanced, then $\left|V_{2}(G)\right|=\sum_{x \in V(G)}\left(3-d_{G}(x)\right) \geq 4-\left|\delta_{G}(V(G))\right|-$ $|\mathcal{U}(G)|=4$ by (S3). Since $G$ is 2 -connected by Claim 3.6.1, $G$ has a circuit containing at least two 2-vertices. Hence $G$ has a removable circuit in either case. It contradicts Claim 3.6.2-(1). Therefore $G$ contains an unbalanced theta.

The existence of unbalanced thetas implies that $\epsilon \in\{-1,1\}$. Let $C$ be an unbalanced circuit. By Claim 3.6.6, $G$ does not contain two disjoint unbalanced circuits, and thus $G-V(C)$ is balanced. By Claim 3.6.2- $(2 \mathrm{~b}), \epsilon \neq \sigma(C)=-1$, so $\epsilon=1$.

Claim 3.6.8. $\left|E_{N}(G)\right| \geq 2$.

Proof of Claim 3.6.8. By Claim 3.6.7, $G$ is unbalanced. Suppose to the contrary that $E_{N}(G)=$ $\left\{e_{0}\right\}$. Let $P$ be the maximal subdivided edge of $G$ containing $e_{0}$. Let $y_{0}, y_{1}$ be the two ends of $P$. Then $\operatorname{Int}(P) \subseteq V_{2}(G)$ and $y_{0}, y_{1} \in V_{3}(G)$. Let $G^{\prime}=G-\operatorname{Int}(P)$ if $\operatorname{Int}(P) \neq \emptyset$; Otherwise, let $G^{\prime}=G-e_{0}$.

We claim that $G^{\prime}$ is 2 -connected. Suppose to the contrary that $G^{\prime}$ is not 2 -connected. Let $B$ be the maximal 2-connected subgraph of $G^{\prime}$ containing $y_{1}$. Since $G=G^{\prime} \cup P$ is 2-connected by Claim 3.6.1, $y_{0} \notin V(B)$ and $\delta_{G^{\prime}}(V(B)) \neq \emptyset$. By the maximality of $B$, each edge in $\delta_{G^{\prime}}(V(B))$ is a cut-edge of $G^{\prime}$. Since $G$ is 2-connected again, $\left|\delta_{G^{\prime}}(V(B))\right|=1$ and thus $\left|\delta_{G}(V(B))\right|=2$ and $B$ is nontrivial since $d_{G}\left(y_{1}\right)=3$. Similarly the maximal 2-connected subgraph of $G^{\prime}$ containing $y_{0}$ is nontrivial and thus contains a circuit. Therefore $B$ is balanced and $G-V(B)$ is balanced and contains circuits since $E_{N}(G)=\left\{e_{0}\right\} \subseteq E(P)$. By Claim 3.6.3, $V(B) \subseteq V_{2}(G)$, which contradicts the fact $y_{1} \in V_{3}(G)$. This proves that $G^{\prime}$ is 2-connected.

(i) $G^{\prime}$ does not contain a circuit $C$ such that $\left\{y_{0}, y_{1}\right\} \cap V(C) \neq \emptyset$ and $\left|V(C) \cap V_{2}(G)\right| \geq 2$. 
Proof of (i). Otherwise, $C$ is a removable circuit such that $G-V(C)$ is balanced and $\sigma(C)=1=\epsilon$ by Claim 3.6.7, a contradiction to Claim 3.6.2-(2b).

Since $G^{\prime}$ is balanced and 2-connected, and is also a shrubbery by Proposition 3.6.1, $\left|V_{2}\left(G^{\prime}\right)\right|=$ $\sum_{x \in V\left(G^{\prime}\right)}\left(3-d_{G^{\prime}}(x)\right) \geq 4$ by (S3) and thus at least two vertices in $V_{2}\left(G^{\prime}\right)$, say $y_{2}$ and $y_{3}$, also belong to $V_{2}(G)$. Note that $\left\{y_{2}, y_{3}\right\} \cap\left\{y_{0}, y_{1}\right\}=\emptyset$. By $(i)$, there is no circuit in $G^{\prime}$ containing $\left\{y_{1}, y_{2}, y_{3}\right\}$. Thus by Theorem 3.6.6, there is a partition of $V\left(G^{\prime}\right)$ into $\mathcal{I}=\left\{X_{1}, X_{2}, Y_{1}, Y_{2}, Y_{3}\right\}$ such that $y_{i} \in Y_{i}(i=1,2,3), \delta_{G^{\prime}}\left(X_{1}, X_{2}\right)=\delta_{G^{\prime}}\left(Y_{i}, Y_{j}\right)=\emptyset(1 \leq i<j \leq 3)$, and $\delta_{G^{\prime}}\left(X_{i}, Y_{j}\right)=$ $e_{i j}(i=1,2 ; j=1,2,3)$. See Figure 3.2. For each $Z \in \mathcal{I}, G^{\prime}[Z]$ is connected since $G^{\prime}$ is 2-connected and $\left|\delta_{G^{\prime}}(Z)\right| \leq 3$.

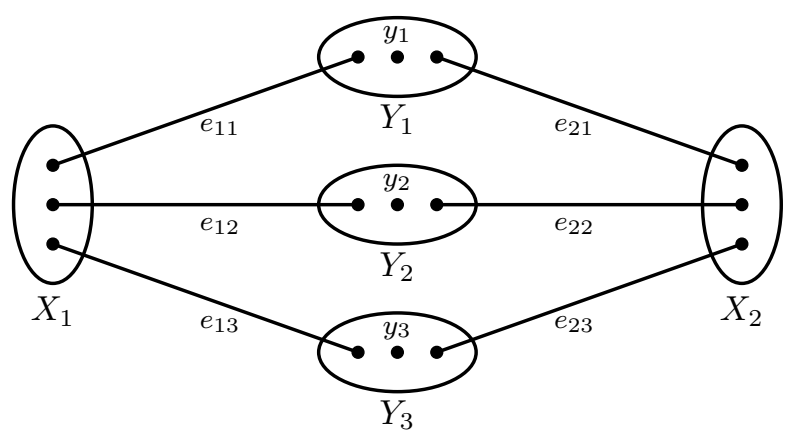

Figure 3.2: A partition of $V\left(G^{\prime}\right)$ into $\mathcal{I}=\left\{X_{1}, X_{2}, Y_{1}, Y_{2}, Y_{3}\right\}$.

Since $G^{\prime}$ is 2 -connected and $\left|\delta_{G^{\prime}}\left(Y_{j}\right)\right|=2$ for $j \in\{2,3\}$, we have the following statement.

(ii) For any $\{i, j\}=\{2,3\}$, there is a circuit $C_{i}$ in $G^{\prime}-Y_{j}$ containing $y_{1}$ and all the edges in $\left\{e_{11}, e_{1 i}, e_{2 i}, e_{21}\right\}$. We choose $C_{i}$ such that $\left|V\left(C_{i}\right) \cap V_{2}(G)\right|$ is as large as possible. Then by (i), $\left|V\left(C_{i}\right) \cap V_{2}(G)\right| \leq 1$.

(iii) $y_{0} \notin Y_{2} \cup Y_{3}, Y_{2}=\left\{y_{2}\right\}$, and $Y_{3}=\left\{y_{3}\right\}$.

Proof of (iii). Let $j \in\{2,3\}$. We first show $\left|Y_{j}\right|=1$ if $y_{0} \notin Y_{j}$. WLOG suppose to the contrary $y_{0} \notin Y_{3}$ and $\left|Y_{3}\right| \geq 2$. Since $G=G^{\prime} \cup P$ and $y_{0} \notin Y_{3},\left|\delta_{G}\left(Y_{3}\right)\right|=\left|\delta_{G^{\prime}}\left(Y_{3}\right)\right|=2$. By $(i i), C_{2}$ is a circuit in $G^{\prime}-Y_{3}$. Since $G^{\prime}[Z]$ is connected for each $Z \in \mathcal{I}, G^{\prime}-Y_{3}$ is connected. Thus there is a $\left(y_{0}, C_{2}\right)$-path $P^{\prime}$ in $G^{\prime}-Y_{3}$, so $P^{\prime} \cup P \cup C_{2}$ is an unbalanced theta in $G-Y_{3}$. Since $G\left[Y_{3}\right]$ is balanced and $\left|\delta_{G}\left(Y_{3}\right)\right|=2$, by Claim 3.6.3, $Y_{3} \subseteq V_{2}(G)$ and $G\left[Y_{3}\right]$ is a path. Thus $Y_{3} \subset V\left(C_{3}\right)$ and $\left|V\left(C_{3}\right) \cap V_{2}(G)\right| \geq 2$, a contradiction to $(i i)$. This proves $\left|Y_{3}\right|=1$. Therefore $\left|Y_{j}\right|=1$ if $y_{0} \notin Y_{j}$ for each $j \in\{2,3\}$.

Now we show $y_{0} \notin Y_{2} \cup Y_{3}$. Otherwise WLOG, assume $y_{0} \notin Y_{3}$ and $y_{0} \in Y_{2}$. Then $Y_{3}=\left\{y_{3}\right\}$ and $y_{3} \in V_{2}(G)$. By (S4), $C_{3}$ is not a balanced 4-circuit, and thus there is a set $Z \in\left\{Y_{1}, X_{1}, X_{2}\right\}$ such that $\left|V\left(C_{3}\right) \cap Z\right| \geq 2$. Since $\left|V(Z) \cap\left\{y_{0}, y_{1}\right\}\right| \leq 1, G[Z]$ is balanced. Obviously $\left|\delta_{G}(Z)\right|=3$. 
By Claim 3.6.4 and the maximality of $\left|V\left(C_{3}\right) \cap V_{2}(G)\right|, C_{3}$ contains a 2-vertex in $Z$. Together with the 2-vertex $y_{3}$, we have $\left|V\left(C_{3}\right) \cap V_{2}(G)\right| \geq 2$, a contradiction to (ii). This shows $y_{0} \notin Y_{2} \cup Y_{3}$ and thus $\left|Y_{2}\right|=\left|Y_{3}\right|=1$.

(iv) $\left|X_{i}\right|=1$ if $y_{0} \notin X_{i}$ for any $i \in\{1,2\}$ and thus $y_{0} \in X_{1} \cup X_{2}$.

Proof of (iv). Suppose that for some $i \in\{1,2\}, y_{0} \notin X_{i}$ and $\left|X_{i}\right| \geq 2$. WLOG assume $i=1$. Let $x_{1 j}$ be the end of $e_{1 j}$ in $X_{1}$ for $j=1,2,3$. Since $\left|X_{1}\right| \geq 2$ and since $\Delta(G)=3$ and $G$ is connected by Claim 3.6.1, $x_{11} \neq x_{1 j}$ for some $j \in\{2,3\}$. Note that $x_{11}, x_{1 j} \in V\left(C_{j}\right)$. Since $\left|\delta_{G}\left(X_{1}\right)\right|=3$ and $G\left[X_{1}\right]$ is balanced, by Claim 3.6.4, there is an $\left(x_{11}, x_{1 j}\right)$-path in $X_{1}$ containing a 2-vertex. So $C_{j}$ contains a 2-vertex in $X_{1}$ by the maximality of $\left|V\left(C_{j}\right) \cap V_{2}(G)\right|$. Since $d_{G}\left(y_{j}\right)=2$ and $C_{j}$ contains $y_{j}, V\left(C_{3}\right)$ contains at least two 2-vertices, a contradiction to $(i i)$. This proves that $\left|X_{i}\right|=1$ if $y_{0} \notin X_{i}$ for any $i \in\{1,2\}$.

If $y_{0} \notin X_{1} \cup X_{2}$, then $\left|X_{1}\right|=\left|X_{2}\right|=1$. By (iii), $G\left[Y_{2} \cup Y_{3} \cup X_{1} \cup X_{2}\right]$ is a balanced 4-circuit, a contradiction to (S4). Therefore $y_{0} \in X_{1} \cup X_{2}$.

By $(i v)$, WLOG assume $y_{0} \in X_{1}$. Then by $(i v)$ and $(i i i),\left|X_{2}\right|=\left|Y_{2}\right|=\left|Y_{3}\right|=1$. Denote $X_{2}=\left\{x_{2}\right\}$.

(v) $Y_{1}=\left\{y_{1}\right\}$.

Proof of $(v)$. Suppose to the contrary that $Y_{1} \neq\left\{y_{1}\right\}$. Then $\left|Y_{1}\right| \geq 2$. Note that $\Delta\left(G^{\prime}\right) \leq$ $\Delta(G)=3$. Since $G^{\prime}$ is 2-connected and $\delta_{G^{\prime}}\left(Y_{1}\right)=\left\{e_{11}, e_{21}\right\}$, the ends of $e_{11}$ and $e_{21}$ in $Y_{1}$ are different. Let $C_{4}$ be a circuit in $G^{\prime}$ containing all the edges in $\left\{e_{11}, e_{12}, e_{22}, e_{21}\right\}$ such that $\left|V\left(C_{4}\right) \cap V_{2}(G)\right|$ is as large as possible. Since $G\left[Y_{1}\right]$ is balanced and $\left|\delta_{G}\left(Y_{1}\right)\right|=3$, with a similar argument in $(i v), C_{4}$ contains a 2 -vertex in $Y_{1}$ and also contains the 2-vertex $y_{2}$. Thus $C_{4}$ contains at least two 2-vertices and hence is removable. Since $\delta_{G}\left(Y_{1}\right) \cap E\left(C_{4}\right)=\left\{e_{11}, e_{21}\right\}$ and $\left|\delta_{G}\left(Y_{1}\right)\right|=3, G-V\left(C_{4}\right)$ is balanced. Since $C_{4}$ does not contain $e_{0}$, the only negative edge, $C_{4}$ is balanced, meaning $\sigma\left(C_{4}\right)=1=\epsilon$, a contradiction to Claim 3.6.2-(2b). This completes the proof of $(v)$.

Let $x_{11}, x_{12}$ and $x_{13}$ be the ends of $e_{11}, e_{12}$ and $e_{13}$ in $X_{1}$, respectively. By (S4), $G\left[\left\{x_{12}, x_{13}, x_{2}, y_{2}, y_{3}\right\}\right]$ is not a 4 -circuit, so $x_{12} \neq x_{13}$. Together with $(i i i)$, (iv), and $(v)$, the structure of $G^{\prime}$ is shown in Figure 3.3.

Now we can complete the proof of Claim 3.6.8.

Recall that $G^{\prime}\left[X_{1}\right]$ is connected. If there is an $\left(x_{12}, x_{13}\right)$-path $P$ in $G^{\prime}\left[X_{1}\right]$ containing $y_{0}$, then $C_{5}=P \cup\left\{e_{12}, e_{22}, e_{23}, e_{13}\right\}$ is a circuit containing $y_{0}$ and two 2-vertices $y_{2}, y_{3}$, a contradiction to $(i)$. Hence by Menger's Theorem, $G^{\prime}\left[X_{1}\right]=G\left[X_{1}\right]$ has a cut-edge separating $y_{0}$ from $\left\{x_{12}, x_{13}\right\}$.

Let $B_{1}$ be the maximal 2-connected subgraphs in $G\left[X_{1}\right]$ containing $y_{0}$. Then every edge in $\delta_{G\left[X_{1}\right]}\left(V\left(B_{1}\right)\right)$ is a cut-edge of $G\left[X_{1}\right]$ by the maximality of $B_{1}$. Since $G\left[X_{1}\right]$ has a cutedge separating $y_{0}$ from $\left\{x_{12}, x_{13}\right\}, x_{12}$ and $x_{13}$ are in the same component, denoted by $B_{2}$, 


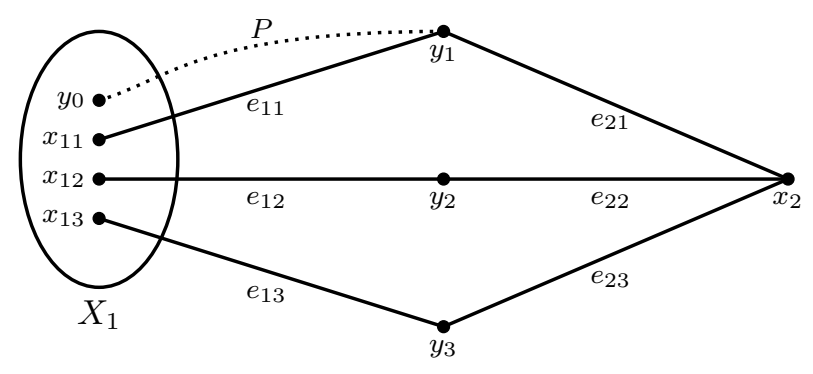

Figure 3.3: $G^{\prime}=G-\operatorname{Int}(P)-E(P)$.

of $G\left[X_{1}\right]-V\left(B_{1}\right)$. Since $G^{\prime}$ is 2-connected and $\delta_{G^{\prime}}\left(X_{1}\right)=\left\{e_{11}, e_{12}, e_{13}\right\}, x_{11} \notin V\left(B_{2}\right)$. Let $\delta_{G\left[X_{1}\right]}\left(V\left(B_{2}\right)\right)=\left\{e^{\prime}\right\}$ and $z$ be the end of $e^{\prime}$ in $B_{2}$. Then there exists an $\left(x_{11}, z\right)$-path $P^{\prime}$ in $G^{\prime}\left[X_{1}\right]$ containing $y_{0}$.

Recall that $x_{12} \neq x_{13}$. WLOG assume $z \neq x_{13}$. Since $\delta_{G}\left(V\left(B_{2}\right)\right)=\left\{e_{12}, e_{13}, e^{\prime}\right\}$ and $B_{2}$ is balanced and has at least two vertices, by Claim $3.6 .4, B_{2}$ has a $\left(z, x_{13}\right)$-path $P^{\prime \prime}$ containing at least one vertex in $V_{2}(G)$. Then $C_{6}=P^{\prime} \cup P^{\prime \prime} \cup x_{13} y_{3} x_{2} y_{1} x_{11}$ is a circuit containing at least two 2-vertices and $y_{0}$, a contradiction to $(i)$. This completes the proof of Claim 3.6.8.

By Claim 3.6.8, $\epsilon(G)=\left|E_{N}(G)\right| \geq 2$. Denote $\epsilon(G)=k$. By Claims 3.6.1 and 3.6.6 and Theorem 3.6.7, we can choose a minimum subset $S \subseteq E(G) \backslash E_{N}(G)$ such that $H=G / S$ satisfies the following properties:

(i) $\Delta(H) \leq 3$;

(ii) $H-E_{N}(H)-\cup_{e \in E_{N}(H)} \operatorname{Int}\left(P_{e}\right)$ is a 2-connected planar graph with a facial circuit $C$, where $P_{e}$ is the maximal subdivided edge in $H$ containing $e$;

(iii) $x_{1}, \ldots, x_{k}, x_{k+1}, \ldots, x_{2 k}$ are pairwise distinct and lie in that cyclic order on $C$, where $E_{N}(H)=E_{N}(G)=\left\{e_{1}, \ldots, e_{k}\right\}$ and $x_{i}, x_{k+i}$ are the two ends of $P_{e_{i}}$ for each $i \in[1, k]$.

For each $v \in V(H)$, let $G_{v}$ denote the corresponding component of $G-E(H)$. Note that $\Delta\left(G_{v}\right) \leq \Delta(G)=3$. By the minimality of $S, G_{v}$ is 2-connected. Otherwise we choose $S \backslash S_{v}$ to replace $S$, where $S_{v}$ is the set of cut-edges of $G_{v}$. Moreover, $S=\cup_{v \in V(H)} E\left(G_{v}\right)$ and $E(G)=$ $E(H) \cup S$.

Claim 3.6.9. $k=2$ and $\left|\operatorname{Int}\left(P_{e_{1}}\right)\right|+\left|\operatorname{Int}\left(P_{e_{2}}\right)\right|=1$.

Proof of Claim 3.6.9. Since $k \geq 2$, it is easy to see $H-\{x\}$ contains an unbalanced theta for any vertex $x$ with $d_{H}(x)=2$. Thus by Claim 3.6.3 and by the minimality of $S$, we have that if $d_{H}(x)=2$ then $G_{x}=\{x\}$.

We construct a circuit $C_{H}$ in the following cases. If there are distinct $i, j \in[1, k]$ such that $\left|\operatorname{Int}\left(P_{e_{i}}\right)\right|=\left|\operatorname{Int}\left(P_{e_{j}}\right)\right|=0$, let $C_{H}=C$; If $\left|\operatorname{Int}\left(P_{e_{i}}\right)\right|+\left|\operatorname{Int}\left(P_{e_{i+1}}\right)\right| \geq 2$ for some $i \in[1, k]$, let 
$C_{H}=C-E\left(x_{i} C x_{i+1}\right)-E\left(x_{i+k} C x_{i+k+1}\right)+P_{e_{i}}+P_{e_{i+1}}$. Note that $G_{v}$ is 2-connected for any $v \in V(H), \Delta(H) \leq 3$ and $\Delta(G)=3$. Then $C_{H}$ can be extended to a removable circuit $C_{G}$ of $G$ such that $\sigma\left(C_{G}\right)=1=\epsilon$ and $G-V\left(C_{G}\right)$ is also balanced, a contradiction to Claim 3.6.2-(2b). This completes the proof of the claim.

WLOG assume that $\operatorname{Int}\left(P_{e_{1}}\right)=\emptyset$ and $\operatorname{Int}\left(P_{e_{2}}\right)=\{y\}$ by Claim 3.6.9. Then $P_{e_{1}}=x_{1} x_{3}$ and $P_{e_{2}}=x_{2} y x_{4}$. Denote $A_{i}=x_{i} C x_{i+1}(\bmod 4)$ for $i \in[1,4], C_{1}=P_{e_{1}} \cup A_{1} \cup P_{e_{2}} \cup A_{3}$, and $C_{2}=P_{e_{1}} \cup A_{4} \cup P_{e_{2}} \cup A_{2}$. Note that both $C_{1}$ and $C_{2}$ contain the 2-vertex $y$. See Figure 3.4.

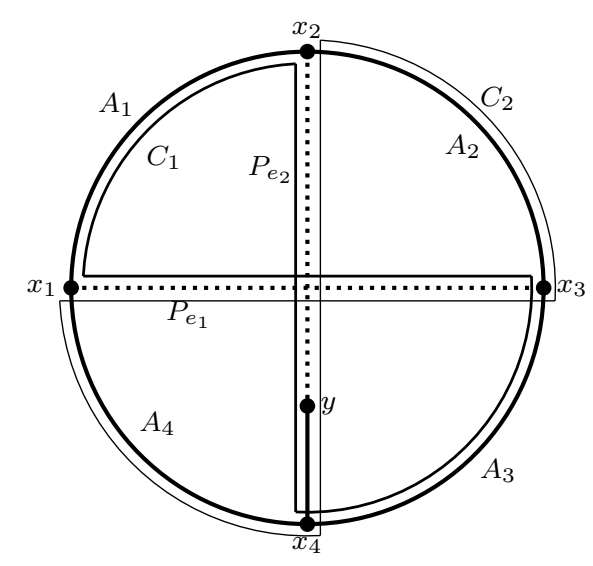

Figure 3.4: $C_{1}$ and $C_{2}$ in $C \cup P_{e_{1}} \cup P_{e_{2}}$.

Claim 3.6.10. $H=G$ and $V_{2}(G)=\{y\}$.

Proof of Claim 3.6.10. As noted in the proof of Claim 3.6.9, for each $x$ with $d_{H}(x)=2$, $G_{x}=\{x\}$. In particular, $G_{y}=\{y\}$.

Note that $G_{x}$ is balanced and $\left|\delta_{G}\left(G_{x}\right)\right| \leq 3$ for every $x \in V(H)$. Thus by Claim 3.6.4, we have the following fact:

(a) If $G_{x}$ is nontrivial, then for each two distinct ends $u, v$ in $V\left(G_{x}\right)$ of $\delta_{G}\left(G_{x}\right)$, there is an $(u, v)$-path in $G_{x}$ containing at least one vertex in $V_{2}$.

Let $x \in V(C)$. WLOG assume $x \in V\left(C_{1}\right)$. Note that if $d_{H}(x)=2$, then $d_{G}(x)=2$. Thus, if $d_{H}(x)=2$ or if $G_{x}$ is nontrivial, $C_{1}$ can be extended to a circuit $C_{1}^{\prime}$ of $G$ such that $C_{1}^{\prime}$ contains the 2-vertex $y$ and one 2-vertex in $G_{x}$ (the latter case follows from (a)). Hence $C_{1}^{\prime}$ is removable, $\sigma\left(C_{1}^{\prime}\right)=1=\epsilon$, and $G-V\left(C_{1}^{\prime}\right)$ is balanced, a contradiction to Claim 3.6.2-(2b). Therefore $d_{H}(x)=3$ and $G_{x}=\{x\}$ for each $x \in V(C)$.

Next we show that $y$ is the only 2-vertex in $G$. Suppose to the contrary that $u$ is a 2vertex in $G$. Then $u \notin V(C)$. Since $G$ is 2-connected, there are two internally disjoint $(u, C)$ paths $Q_{1}$ and $Q_{2}$ in $G$ with $v_{1}$ and $v_{2}$ the end vertices in $C$ respectively. Since $\Delta(G)=3$, $v_{1} \neq v_{2}$. Let $C_{3}=Q_{1} \cup Q_{2} \cup v_{1} C v_{2}$ and $C_{4} \in\left\{C_{1}, C_{2}\right\}$ such that $V\left(C_{4}\right) \cap\left\{v_{1}, v_{2}\right\} \neq \emptyset$. Then 
$C^{\prime}=C_{3} \Delta C_{4}$ is a circuit containing two 2-vertices $\{y, u\}$ and the two negative edges. Thus $C^{\prime}$ is removable, $\sigma\left(C_{1}^{\prime}\right)=1=\epsilon$, and $G-V\left(C^{\prime}\right)$ is balanced, which contradicts Claim 3.6.2-(2b). Thus $V_{2}(G)=\{y\}$.

Since $V_{2}(G)=\{y\}, G_{x}$ is trivial by (a). Therefore $H=G$.

Claim 3.6.11. Int $\left(A_{i}\right) \neq \emptyset$ for each $i \in[1,4]$.

Proof of Claim 3.6.11. Suppose to the contrary that $\operatorname{Int}\left(A_{i}\right) \neq \emptyset$ for some $i \in[1,4]$. WLOG assume $\operatorname{Int}\left(A_{1}\right)=\emptyset$. Then $A_{1}$ is a chord in $\mathcal{U}\left(C_{2}\right)$. Since $C_{2}$ contains the 2-vertex $y, C_{2}$ is removable, which contradicts Claim 3.6.2-(2b) since $\sigma\left(C_{2}\right)=1=\epsilon$ and $G-V\left(C_{2}\right)$ is balanced.

\section{The final step.}

By Claim 3.6.11, let $y_{1} \in \operatorname{Int}\left(A_{1}\right)$ be the neighbor of $x_{1}$. Let $Q$ be the component of $G-E(C)$ containing $y_{1}$. Since $d_{G}\left(y_{1}\right)=3$ by Claim 3.6.10, $Q$ is nontrivial. Obviously, $V(Q) \cap$ $\left\{x_{1}, x_{2}, x_{3}, x_{4}\right\}=\emptyset$ since $\Delta(G)=3$.

If there is a vertex $y_{2}$ in $V(Q) \cap\left(\operatorname{Int}\left(A_{2}\right) \cup \operatorname{Int}\left(A_{3}\right)\right)$, let $P$ be a $\left(y_{1}, y_{2}\right)$-path in $Q$. Since $\Delta(G) \leq 3, C_{3}=P \cup y_{1} C y_{2}$ is a circuit containing $x_{2}$. Then $C^{\prime}=C_{2} \triangle C_{3}$ is a circuit of $G$ containing $y$ and the chord $x_{1} y_{1} \in \mathcal{U}\left(C^{\prime}\right)$. Thus $C^{\prime}$ is a removable circuit of $G$, a contradiction to Claim 3.6.2-(2b) since $G-V\left(C^{\prime}\right)$ is balanced.

If $V(Q) \cap\left(\operatorname{Int}\left(A_{2}\right) \cup \operatorname{Int}\left(A_{3}\right)\right)=\emptyset$, then $V(Q) \cap V(C) \subseteq \operatorname{Int}\left(A_{4}\right) \cup \operatorname{Int}\left(A_{1}\right)$. Note that $|V(Q) \cap V(C)| \geq 2$ since $G$ is 2-connected. Let $y_{2}, y_{3} \in V(Q) \cap V(C)$ be two ends of a segment $P^{\prime}$ of $A_{4} \cup A_{1}$ such that the length of $P^{\prime}$ is as large as possible. By Claim 3.6.10, $G^{\prime}=G-x_{1} x_{3}-y$ is a 2-connected planar graph with a facial circuit $C$, and so $T^{\prime}=\delta_{G^{\prime}}\left(V\left(P^{\prime}\right)\right) \cap E(C)$ is a 2edge-cut of $G^{\prime}$. Let $T=T^{\prime}$ if $y_{2}, y_{3} \in \operatorname{Int}\left(A_{1}\right)$, and otherwise $T=T^{\prime} \cup\left\{x_{1} x_{3}\right\}$. Then $T$ is an edge-cut of $G$ with $|T| \leq 3$ and the component, denoted by $R$, of $G-T$ containing $y_{2}$ is balanced and doesn't contain $y$. Since $\left|\delta_{G}(V(R))\right|=|T| \leq 3$, by $(\mathrm{S} 3), \sum_{v \in V(R)}\left(3-d_{G}(v)\right) \geq$ $4-\left|\delta_{G}(V(R))\right|-2|\mathcal{U}(R)| \geq 1$, and so this component $R$ contains a 2-vertex (distinct from $y$ ), which contradicts $V_{2}(G)=\{y\}$ by Claim 3.6.10. This completes the proof of Lemma 3.6.10.

\subsubsection{Completing the proof of Theorem 3.1.3}

Finally we are to complete the proof of Theorem 3.1.3 in this subsection.

By Lemma 3.6.5, it suffices to show that every cubic shrubbery $G$ admits a balanced $\mathbb{Z}_{2} \times \mathbb{Z}_{3^{-}}$ NZF. If $G$ is balanced, then such a flow exists by Theorem 3.6.1.

Assume that $G$ is unbalanced. We claim that $G$ contains either an unbalanced theta or a negative loop.

If $G$ is 2-connected, then for any unbalanced circuit $C$, we can easily find a path in $G-E(C)$ to connect two distinct vertices of $V(C)$, and thus $G$ has an unbalanced theta. 
If $G$ is not 2-connected, then it has an cut-edge since $G$ is cubic. Let $B$ be a leaf block of $G$. If $B$ is trivial, then $B$ is a negative loop. If $B$ is nontrivial, then $B$ is unbalanced by Proposition 3.2 .1 since $G$ is flow-admissible by (S2). Since $B$ is 2-connected and all vertex except one has degree 3 , similar to the argument in the case when $G$ is 2 -connected, one can find an unbalanced theta in $B$, which is also an unbalanced theta in $G$.

By the claim, we apply Lemma 3.6 .10 on cubic shrubbery $G$ with $\varepsilon=1$ to obtain an NZF $f=\left(f_{1}, f_{2}\right)$ with $\sigma\left(\operatorname{supp}\left(f_{1}\right)\right)=\varepsilon=1$. By Definition 3.6.1 this is a balanced $\mathbb{Z}_{2} \times \mathbb{Z}_{3}$-NZF as desired. This completes the proof of Theorem 3.1.3. 


\section{Bibliography}

[1] B. Alspach, L. Goddyn, and C.-Q. Zhang. Graphs with the circuit cover property. Trans. Amer. Math. Soc., 344(1) (1994), 131-154.

[2] B. Alspach and C.-Q. Zhang. Cycle covers of cubic multigraphs. Discrete Math., 111(1-3) (1993), 11-17. Graph theory and combinatorics (Marseille-Luminy, 1990).

[3] J. A. Bondy and U. S. R. Murty, Graph theory with applications, Macmillan, London, 1976.

[4] J. A. Bondy and U. S. R. Murty. Graph theory, volume 244 of Graduate Texts in Mathematics. Springer, New York, 2008.

[5] A. Bouchet, Nowhere-zero integral flows on a bidirected graph, J. Combin. Theory Ser. B., 34 (1983), 279-292.

[6] J. Cheng, Y. Lu, R. Luo and C.-Q. Zhang, Signed graphs: from modulo flows to integervalued flows, SIAM J. Discrete Math., 32 (2018), 956-965.

[7] R. Diestel. Graph theory, volume 173 of Graduate Texts in Mathematics. Springer, Heidelberg, fourth edition, 2010.

[8] M. N. Ellingham. Petersen subdivisions in some regular graphs. In Proceedings of the fifteenth southeastern conference on combinatorics, graph theory and computing (Baton Rouge, La., 1984), volume 44 (1984), 33-40.

[9] G. Fan and C.-Q. Zhang. Circuit decompositions of Eulerian graphs. J. Combin. Theory Ser. B, 78(1) (2000), 1-23.

[10] H. Fleischner, Eine gemeinsame Basis für die Theorie der eulerschen Graphen und den Satz von Petersen. Monatsh. Math. 81 (1976), 267-278.

[11] H. Fleischner. Eulersche Linien und Kreisüberdeckungen, die vorgegebene Durchgänge in den Kanten vermeiden. J. Combin. Theory Ser. B, 29(2) (1980), 145-167. 
[12] H. Fleischner. Eulerian graphs. In Selected topics in graph theory, 2, pages 17-53. Academic Press, London, 1983.

[13] H. Fleischner and A. Frank. On circuit decomposition of planar Eulerian graphs. J. Combin. Theory Ser. B, 50(2) (1990), 245-253.

[14] H. Fleischner, Personal communication, 1990's.

[15] J. Hägglund and A. Hoffmann-Ostenhof. Construction of permutation snarks. J. Combin. Theory Ser. B, 122 (2017), 55-67.

[16] A. Itai and M. Rodeh. Covering a graph by circuits. In Automata, languages and programming (Fifth Internat. Colloq., Udine, 1978), volume 62 of Lecture Notes in Comput. Sci., pages 289-299. Springer, Berlin-New York, 1978.

[17] F. Jaeger, On nowhere-zero flows in multigraphs, Proceedings of the Fifth British Combinatorial Conference 1975, Congr. Numer. 15 (1975), 373-378.

[18] F. Jaeger, Flows and generalized coloring theorems in graphs, J. Combin. Theory Ser. B, 26 (1979), 205-216.

[19] F. Jaeger, N. Linial, C. Payan and M. Tarsi, Group connectivity of graphs - A nonhomogeneous analogue of nowhere-zero flow properties, J. Combin. Theory Ser. B., 56 (1992), 165-182.

[20] H.-J. Lai and C.-Q. Zhang. Hamilton weights and Petersen minors. J. Graph Theory, 38(4) (2001), 197-219.

[21] Y. Lu, R. Luo and C.-Q. Zhang, Multiple weak 2-linkage and its applications on integer flows on signed graphs, European J. Combin. 69 (2018), 36-48.

[22] Mohar, B., and Thomassen, C., Graphs on Surfaces, (2001) Johns Hopkins University Press.

[23] James R. Munkres, Topology, Second Edition, 2000.

[24] P. D. Seymour. Sums of circuits. In Graph theory and related topics (Proc. Conf., Univ. Waterloo, Waterloo, Ont., 1977), pages 341-355. Academic Press, New York-London, 1979.

[25] P. D. Seymour, Nowhere-zero 6-flows, J. Combin. Theory Ser. B, 30 (1992), 130-135.

[26] P. D. Seymour and K. Truemper. A Petersen on a pentagon. J. Combin. Theory Ser. B, 72(1) (1998), 63-79.

[27] G. Szekeres. Polyhedral decompositions of cubic graphs. Bull. Austral. Math. Soc., 8 (1973), $367-387$. 
[28] W. T. Tutte, A contribution to the theory of chromatic polynomials, Canadian J. Math. 6 (1954), 80-91.

[29] W. T. Tutte, On the algebraic theory of graph colorings, J. Combin. Theory, 1 (1966), $15-50$.

[30] W.T. Tutte. Personal correspondence with H. Fleischner. July 22, 1987.

[31] M.E. Watkins and D.M. Mesner, Some theorems about $n$-vertex connected graphs, Canad. J. Math., 19 (1967), 1319-1328.

[32] D. B. West. Introduction to graph theory. Prentice Hall, Inc., Upper Saddle River, NJ, 1996.

[33] R. Xu and C.-Q. Zhang, On flows in bidirected graphs, Discrete Math., 299 (2005), 335-343.

[34] C.-Q. Zhang. Hamiltonian weights and unique 3-edge-colorings of cubic graphs. J. Graph Theory, 20(1) (1995), 91-99.

[35] C.-Q. Zhang, Integer flows and cycle covers of graphs, Marcel Dekker, New York, 1997.

[36] C.-Q. Zhang. Cycle covers (I) - minimal contra pairs and Hamilton weights. J. Combin. Theory Ser. B, 100(5) (2010), 419-438.

[37] C.-Q. Zhang, Circuit double cover of graphs, Cambridge University Press, 2012.

[38] C.-Q. Zhang. Cycle covers (II) - Circuit chain, Petersen chain and Hamilton weights. J. Combin. Theory Ser. B, 120 (2016), 36-63.

[39] O. Zýka, Nowhere-zero 30-flow on bidirected graphs, Thesis, Charles University, Praha, KAM-DIMATIA, Series 87-26 (1987). 